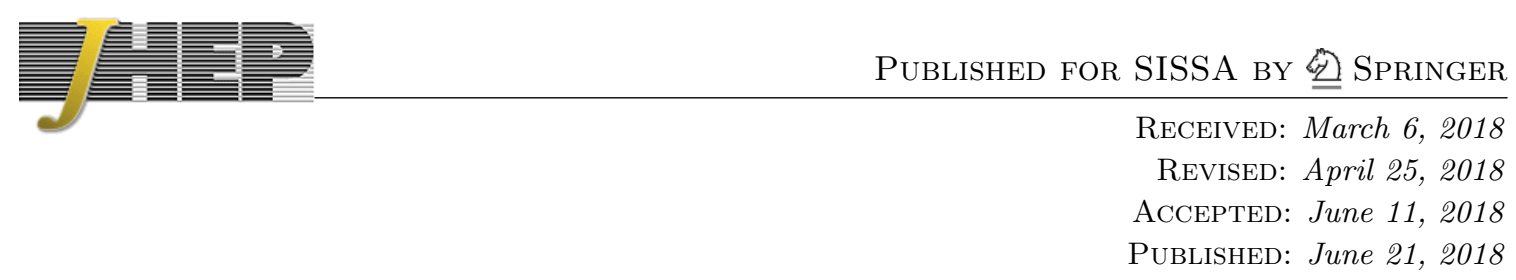

\title{
Searching for metastable particles with sub-millimeter displaced vertices at hadron colliders
}

\author{
Hayato Ito, ${ }^{a}$ Osamu Jinnouchi, ${ }^{b}$ Takeo Moroi $^{a}{ }^{a}$ Natsumi Nagata ${ }^{a}$ \\ and Hidetoshi Otono ${ }^{c}$ \\ ${ }^{a}$ Department of Physics, University of Tokyo, \\ Tokyo 113-0033, Japan \\ ${ }^{b}$ Department of Physics, Tokyo Institute of Technology, \\ Tokyo 152-8551, Japan \\ ${ }^{c}$ Research Center for Advanced Particle Physics, Kyushu University, \\ Fukuoka 819-0395, Japan \\ E-mail: ito@hep-th.phys.s.u-tokyo.ac.jp, jinnouchi@phys.titech.ac.jp, \\ moroi@phys.s.u-tokyo.ac.jp, natsumi@hep-th.phys.s.u-tokyo.ac.jp, \\ otono@phys.kyushu-u.ac.jp
}

ABSTRACT: A variety of new-physics models predict metastable particles whose decay length is $\lesssim 1 \mathrm{~mm}$. Conventional displaced-vertex searches are less sensitive to this submillimeter decay range, and thus such metastable particles have been looked for only in usual prompt decay searches. In this paper, we show that an additional event-selection cut based on the vertex reconstruction using charged tracks considerably improves the sensitivity of ordinary searches which rely only on kinematic selection criteria, for particles with a decay length of $\gtrsim 100 \mu \mathrm{m}$. To that end, we consider a metastable gluino as an example, and study the impact of this new event-selection cut on gluino searches at the LHC by simulating both the signal and Standard Model background processes. Uncertainty of the displaced-vertex reconstruction due to the limited resolution of track reconstruction is taken into account. We also discuss possibilities for optimization of the kinematic selection criteria, which takes advantage of significant reduction of background through the requirement of displaced vertices. In addition, we demonstrate that using the method discussed in this paper it is possible to measure the lifetime of metastable particles with an $\mathcal{O}(1)$ accuracy at the high-luminosity LHC. Implications for a future $100 \mathrm{TeV}$ collider are also studied, where produced particles tend to be more boosted and thus it is easier to detect the longevity of metastable particles.

Keywords: Supersymmetry Phenomenology

ARXIV EPRINT: 1803.00234 


\section{Contents}

1 Introduction 1

2 Vertex reconstruction method 3

3 Gluino phenomenology 5

$\begin{array}{llll}4 & 13 & \text { TeV LHC } & 7\end{array}$

4.1 MC simulation 8

$\begin{array}{llr}4.2 & \text { Event selection criteria } & 12\end{array}$

$\begin{array}{lll}4.3 & \text { Prospects } & 16\end{array}$

4.4 Lifetime measurements 24

$5 \quad 100$ TeV collider $\quad 25$

5.1 MC simulation 25

$\begin{array}{lll}5.2 & \text { Event selection } & 28\end{array}$

5.3 Prospects 30

6 Conclusion and discussion $\quad 35$

\section{Introduction}

Many models beyond the Standard Model (SM) predict the existence of long-lived particles [1]. For instance, in supersymmetric (SUSY) models, gluino tends to be long-lived if sfermion masses are in the multi-TeV range [2-4]. Such a long-lived particle is also found in the gauge-mediation models [5-8], in $R$-parity violating models [9-11], in SUSY axion models [12-18], in the stealth SUSY scenario [19-21], in the SUSY relaxion models [22, 23], and in a degenerate SUSY spectrum [24-34]. Other well-motivated scenarios such as Neutral Naturalness [35-39], hidden-valley models [40-44], composite Higgs models [45], dark matter models [46-49], and neutrino models [50-59] also provide metastable particles, and the searches of these particles offer a promising way of testing these models. The predicted lifetime of these particles spreads over a wide range; some particles are predicted to decay much before they reach the detector region, while others can be regarded as stable particles at collider experiments. Therefore it is important to develop various strategies to cover the whole potential signatures of long-lived particles, which depend on the lifetime of these particles as well as their decay properties. Indeed, a lot of efforts have been dedicated to searching for long-lived particles at the LHC in a variety of search channels, including displaced-vertex searches [60-67], disappearing track searches [68-70], the searches for large energy-loss signatures [71-75], and so on. Null results in these searches have imposed stringent limits on long-lived particles. 
Most of these searches, however, have sensitivities only to particles with a proper decay length of $c \tau \gtrsim 1 \mathrm{~mm}$, where $\tau$ is the proper lifetime and $c$ is the speed of light. ${ }^{1}$ Particles with a shorter decay length have been probed only with ordinary prompt-decay searches. Although these prompt searches do give strong limits on such particles, the ignorance of the lifetime information leaves room for improvement in these searches. Given the null result so far, it is desirable to pursue every possibility of potential improvements by fully utilizing the ability of the detectors at the LHC.

Motivated by this observation, in ref. [78], we proposed a new event-selection criterion based on the reconstruction of displaced vertices using charged tracks associated with the decay points. These vertices are reconstructed in the same way as those used for the primary vertex reconstruction, which makes it possible to determine the vertex position with a resolution of $\mathcal{O}(10) \mu \mathrm{m}$. We then found that the reach of ordinary prompt-decay searches could be significantly extended for metastable particles with $c \tau \gtrsim 100 \mu \mathrm{m}$ when we require this new event-selection cut in addition to other existing selection criteria. Moreover, the reconstruction of displaced vertices allows us to measure the lifetime of metastable particles.

The aim of the present paper is to elaborate on this idea with the help of detailed Monte Carlo (MC) collider simulations. To be concrete, we here focus on metastable gluino searches, but a similar study can also be performed for other metastable particles. With the generation of SM background processes, we reevaluate the efficiency of the cut proposed in ref. [78] and confirm that this is able to separate signal events from background quite efficiently for particles with $c \tau \gtrsim 100 \mu \mathrm{m}$. We also discuss optimization of a kinematical selection cut in order to make the most of the background-reduction ability of this new cut. It is then found that the reach of prompt-decay searches can considerably be extended with this method for particles with $100 \mu \mathrm{m} \lesssim c \tau \lesssim 100 \mathrm{~mm}$. The improvement in the sensitivity is especially significant when the parent and daughter particles have masses close to each other. We also find that the lifetime of metastable particles can be measured with an $\mathcal{O}(1)$ accuracy at the high-luminosity LHC.

The selection method discussed in this paper can in principle be applied to any collider experiments where high tracking performance is implemented. We thus study the prospects of our method for a future $100 \mathrm{TeV} p p$ collider $[79,80]$ on the assumption that charged tracks are reconstructed with an accuracy similar to that obtained at the LHC. We see that the extent of the resultant improvement in the sensitivity in this case is rather large compared with the $13 \mathrm{TeV}$ LHC case, since gluinos at a $100 \mathrm{TeV}$ collider are produced in a highly boosted state so that their flight distance tends to be prolonged. This study suggests that it is desirable to develop a detector system at a future $100 \mathrm{TeV}$ collider which has a good capability for track and vertex reconstruction.

This paper is organized as follows. In the next section, we briefly review the vertex reconstruction method used in our analysis, which is discussed in more detail in ref. [78]. As we mentioned above, in our analysis we consider metastable gluinos as a concrete example;

\footnotetext{
${ }^{1}$ We however note that there are several exceptions, i.e., those which are sensitive to $c \tau \lesssim 1 \mathrm{~mm}[62$, $66,76,77]$; these searches are focused on rather specific signatures and, in particular, unable to be directly applied to the setup we consider in this paper.
} 
we thus summarize the properties of metastable gluinos in section 3 with some discussions on theoretical motivations for such particles. Then, we show the results of our analysis for the $13 \mathrm{TeV}$ LHC and a future $100 \mathrm{TeV}$ collider in section 4 and section 5, respectively. Section 6 is devoted to conclusion and discussions.

\section{Vertex reconstruction method}

To begin with, we describe the vertex reconstruction procedure we use in the following analysis. We also discuss how to take account of the deterioration in the vertex reconstruction caused by the limited resolution of track reconstruction. See ref. [78] for more detailed explanation of this procedure and the validation of our method.

In the following discussion, we focus on the event topology in which metastable particles are pair-produced and their decay product contains a large number of charged particles as well as a stable neutral massive particle, which yields a large missing transverse energy. Such an event topology is realized in gluino decays, where each decay vertex is accompanied with two hard jets as we see in section 3. By using the information of tracks associated with the charged particles emitted from the decay points (such as those in the jets in the case of gluino decay), we can determine the positions of the decay points of metastable particles. In particular, if a metastable particle is pair-produced, two decay vertices exist. Observation of two distinct decay vertices is a strong evidence of a new metastable particle like long-lived gluino. In the following analysis, we propose to use the distance between two reconstructed decay vertices as a discriminator to reduce SM backgrounds.

The procedure to reconstruct vertices emitting hadronic activities has been well established, as it is used for the primary-vertex reconstruction by both the ATLAS [81-83] and CMS $[84,85]$ collaborations. We propose to apply such a procedure to the search of metastable particles. Typical resolution of the primary vertex position is $\mathcal{O}(10) \mu \mathrm{m}$, which indicates that the decay positions of metastable particles can also be determined with a similar precision if the number of charged tracks associated with the decay vertices is sufficiently large, which is expected to be realized in gluino decay events.

In our analysis, we reconstruct vertices from charged tracks using the adaptive vertex fitting algorithm [86], which is adopted in refs. [81, 82]. In this algorithm, we first obtain a set of crossing points of the tracks; each crossing point is defined as the midpoint between the two points of closest approach of two tracks. A vertex seed is then determined from these crossing points using a method called the fraction of sample mode with weights [87], where the vertex position is set to be the point of highest density of the crossing points (this is performed separately in each spatial coordinate). We denote the position vector of this vertex by $\boldsymbol{v}$. Next, we assign a weight $w_{i}$ to each track (labeled by $i$ ) such that tracks which lie distant from the vertex seed $\boldsymbol{v}$ are down-weighted. This weight contains a parameter that controls the reduction factor of weights for distant tracks. See ref. [78] for the explicit form of $w_{i}$. We then find another vertex which minimizes the sum of the squared standardized distances from the vertex seed $\chi_{i}^{2}(\boldsymbol{v})$,

$$
\chi_{i}^{2}(\boldsymbol{v}) \equiv \frac{d_{i}^{2}(\boldsymbol{v})}{\sigma_{d_{0}}^{2}+\sigma_{z_{0} \sin \theta}^{2}},
$$


with $d_{i}(\boldsymbol{v})$ being the distance from the vertex $\boldsymbol{v}$, multiplied by the weights $w_{i}$ (we refer to this as the weighted vertex chi square value, $\left.\chi_{w}^{2} \equiv \sum_{i} w_{i} \chi_{i}^{2}(\boldsymbol{v})\right)$, and regard this vertex as the new vertex seed. This process is iterated while changing the parameter in the weights so that the distant tracks are more highly down-weighted, until the change in the parameter is stopped and the convergence of the vertex position is reached within a certain accuracy (we take this to be $1 \mu \mathrm{m}$ ). With the help of the parameter in the weight, we can gradually narrow down the range of the tracks we use to obtain a vertex candidate, through which we can avoid being stuck at a local minimum of the weighted vertex chi square value. For the choice of the parameters and the procedure of the iteration, we follow refs. $[78,86]$.

The resolution of the vertex reconstruction strongly depends on that of the track reconstruction. To take this effect into account, we smear tracks obtained from the MC-truth information by shifting each track in parallel by impact parameters which are randomly chosen from Gaussian distributions with the variances set by impact parameter resolutions. To be specific, we refer to the performance of the ATLAS detector in what follows. The tracking performance of the ATLAS inner detector for the $13 \mathrm{TeV}$ run is given in refs. $[88,89]$. Here we only consider the resolution of the transverse and longitudinal impact parameters, $d_{0}$ and $z_{0} \sin \theta$, respectively, as the resolution for the track direction is sufficiently small [90]. ${ }^{2}$ The impact parameter resolutions are parametrized as functions of the transverse momentum $p_{\mathrm{T}}[90]$ :

$$
\sigma_{X}\left(p_{\mathrm{T}}\right)=\sigma_{X}(\infty)\left(1 \oplus p_{X} / p_{\mathrm{T}}\right)
$$

for $X=d_{0}, z_{0} \sin \theta$, where $\sigma_{X}(\infty)$ and $p_{X}$ are some constant parameters determined via the fitting of this expression onto the experimental result obtained by the ATLAS collaboration $[88,89]$. We here neglect the $\eta$-dependence of $\sigma_{X}$ as it turns out to be sufficiently small for $p_{\mathrm{T}} \gtrsim$ a few $\mathrm{GeV}[90,91]$.

The validation of the above procedure was performed in ref. [78] using minimum-bias event samples, where the position of primary vertices are reconstructed with our method using smeared tracks. The vertex resolution obtained in this way was found to be in good agreement with those given in ref. [83]. We thus use this prescription to reconstruct displaced vertices associated with the decay of metastable particles in what follows.

As mentioned above, gluinos are pair produced and the decay of each gluino gives rise to two hard jets; this event topology is illustrated in figure 1. Considering this, we reconstruct displaced vertices in the metastable gluino decay events by using only tracks associated with four high- $p_{\mathrm{T}}$ jets. The way of choosing these four high- $p_{\mathrm{T}}$ jets, as well as the track conditions, will be given in section 4 . The reconstruction of the decay vertices in gluino decay events is complicated due to the fact that it is unclear which pair of jets is associated with the same vertex. In our analysis, we consider all three possible patterns of pairings out of the four jets and reconstruct a vertex for each pair using the method described above. For each pairing, we determine the positions of two decay vertices assuming that the jets in a pair originate from the same decay vertex; each decay vertex is determined with the

\footnotetext{
${ }^{2}$ The polar angle $\theta$ is defined with respect to the $z$-axis, which lies in the direction of the beam axis. $d_{0}$ and $z_{0}$ denote the distance of the closest approach between the track and the beam axis and its $z$-coordinate, respectively.
} 


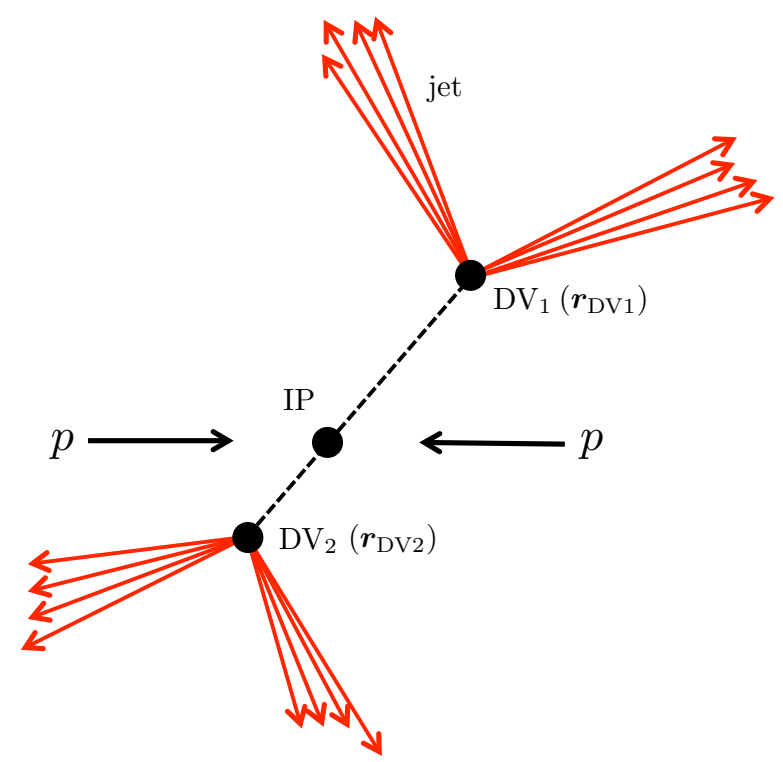

Figure 1. The event topology of metastable gluino decay. Here, $\mathrm{DV}_{1}$ and $\mathrm{DV}_{2}$ are two decay vertices of gluino, while IP is the interaction point.

tracks associated to the jets constituting the pair. We then choose the pairing that has the smallest value of $\chi^{2}$ defined by

$$
\chi^{2} \equiv \frac{\sum_{i \in \operatorname{trk}\left(\boldsymbol{v}_{1}\right)} w_{i} \chi_{i}^{2}\left(\boldsymbol{v}_{1}\right)+\sum_{j \in \operatorname{trk}\left(\boldsymbol{v}_{2}\right)} w_{j} \chi_{j}^{2}\left(\boldsymbol{v}_{2}\right)}{\sum_{i \in \operatorname{trk}\left(\boldsymbol{v}_{1}\right)} w_{i}\left(\boldsymbol{v}_{1}\right)+\sum_{j \in \operatorname{trk}\left(\boldsymbol{v}_{2}\right)} w_{j}\left(\boldsymbol{v}_{2}\right)},
$$

where $\operatorname{trk}\left(\boldsymbol{v}_{1,2}\right)$ denotes the set of tracks associated with the vertex reconstructed at the position $\boldsymbol{v}_{1,2}$ for each pair of jets. Note that $\chi_{i}^{2}(\boldsymbol{v})$ is the squared standardized distance from the vertex $\boldsymbol{v}$, which is defined by eq. (2.1). We employ the vertices obtained with this pairing, and denote their positions by $\boldsymbol{r}_{\mathrm{DV} 1,2} \equiv \boldsymbol{v}_{1,2}$. This new information of the position of the displaced vertices will be used to improve the metastable gluino searches in the following analysis.

\section{Gluino phenomenology}

As mentioned in the introduction, we consider a metastable gluino as a concrete example and study the effect of the new selection cut on the gluino searches. Here we assume that all of the squarks are much heavier than gluino and there is at least one neutralino which is lighter than the gluino. In this case, gluinos $\tilde{g}$ decay either in the tree-level three-body decay processes with the emission of a pair of quarks and a neutralino $\tilde{\chi}^{0}$ or chargino $\tilde{\chi}^{ \pm}, \tilde{g} \rightarrow q \bar{q} \tilde{\chi}^{0}, q \bar{q}^{\prime} \tilde{\chi}^{ \pm}$, or in the one-loop two-body decay process where a gluino and a neutralino is emitted: $\tilde{g} \rightarrow g \tilde{\chi}^{0}$. It is found that if the gluino mass is $\gtrsim 2 \mathrm{TeV}$, then the three-body decay processes dominate the two-body decay [2-4]. As the three-body decay processes are induced by the exchange of virtual heavy squarks, the amplitudes of these processes are suppressed by the squared masses of these heavy squarks, $m_{\tilde{q}}^{2}$. As a 
consequence, gluinos become long-lived if the squark masses are sufficiently heavy. To see this more quantitatively, we show an approximate formula for the decay length of gluinos:

$$
c \tau_{\tilde{g}} \simeq 200 \times\left(\frac{m_{\tilde{q}}}{10^{3} \mathrm{TeV}}\right)^{4}\left(\frac{m_{\tilde{g}}}{2 \mathrm{TeV}}\right)^{-5} \mu \mathrm{m},
$$

where $m_{\tilde{g}}$ is the mass of gluino. In deriving this approximate formula, we have assumed that the gluino mainly decays into the first-generation quarks, the masses of bino and wino are much smaller than the gluino mass, and higgsinos are heavier than the gluino. In this case, $m_{\tilde{q}}$ corresponds to the mass of the first-generation squarks. From eq. (3.1), we see that metastable gluinos with a decay length of $\gtrsim 100 \mu \mathrm{m}$ are obtained if squarks lie around the $\mathrm{PeV}$ scale or higher.

In fact, the PeV-scale squarks are phenomenologically well motivated. First and foremost, with such heavy squarks (especially heavy stops), the observed value of the SM-like Higgs boson mass [92] can be explained within the minimal SUSY SM [93-97]. In addition, the contributions of SUSY particles to the flavor-changing neutral current processes, the electric dipole moments, and the dimension-five proton decay processes are all suppressed by heavy sfermion masses, and thus the experimental limits on such dangerous processes can easily be evaded [98-105]. Even though squarks are as heavy as $\mathcal{O}(1) \mathrm{PeV}$, fermionic SUSY particles can naturally be around the $\mathrm{TeV}$ scale since their masses are protected by chiral symmetry. A simple framework to realize this split spectrum [106-111] is provided by the so-called anomaly-mediated SUSY-breaking mechanism [106, 112], ${ }^{3}$ where gaugino masses are induced by quantum corrections and thus suppressed by a loop factor compared with the gravitino mass. This gives theoretical support for the split SUSY mass spectrum. A distinguished feature of this type of spectrum is that the lightest SUSY particle (LSP), which is one of the TeV-scale fermionic SUSY particles such as the neutral wino and higgsino, can be a promising candidate for dark matter in the Universe [113, 114]. These advantages have thus attracted a lot of attentions [115-122] especially after the early stage of the LHC run. In such a split spectrum, gluino may also lie around the $\mathrm{TeV}$ scale and be within the reach of the LHC and/or a future hadron collider. Gluinos in this case are expected to have a sizable decay length according to eq. (3.1) - in this sense, a metastable gluino may be regarded as a smoking-gun signature of the split-type SUSY spectrum and the detection of such a gluino enables us to confirm this scenario experimentally. Moreover, since the lifetime of metastable gluinos reflects the squark mass scale, we may probe this via the precise measurements of the gluino decay length, even though we cannot produce squarks directly at colliders. We discuss this possibility in more detail in section 4.4.

\footnotetext{
${ }^{3}$ In the anomaly mediation, the gluino mass is proportional to the gravitino mass with its proportional constant given by the strong gauge coupling beta function. Under this relation, if both gravitino and squarks are around the $\mathrm{PeV}$ scale, then the gluino mass tends to be much larger than the TeV scale. This consequence can be evaded if the SUSY-breaking effects are transmitted to the visible sector below the Planck scale; in this case, squark masses are larger than the gravitino mass, and thus it is possible to obtain a $\mathrm{TeV}$-scale gluino and $\mathrm{PeV}$-scale squarks simultaneously, by assuming a gravitino mass lower than the $\mathrm{PeV}$ scale. Keeping this in mind, we consider both the gluino mass and squark masses, and thus the gluino lifetime as well, to be free parameters in the following discussion.
} 


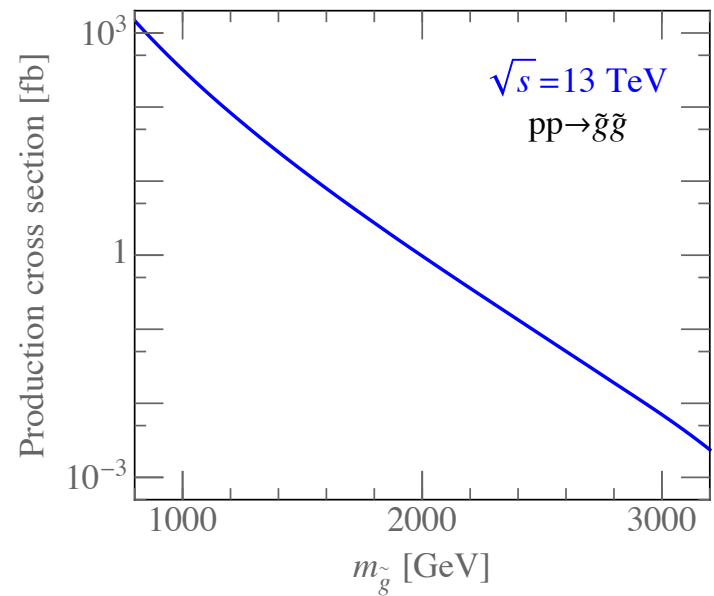

(a) $13 \mathrm{TeV}$

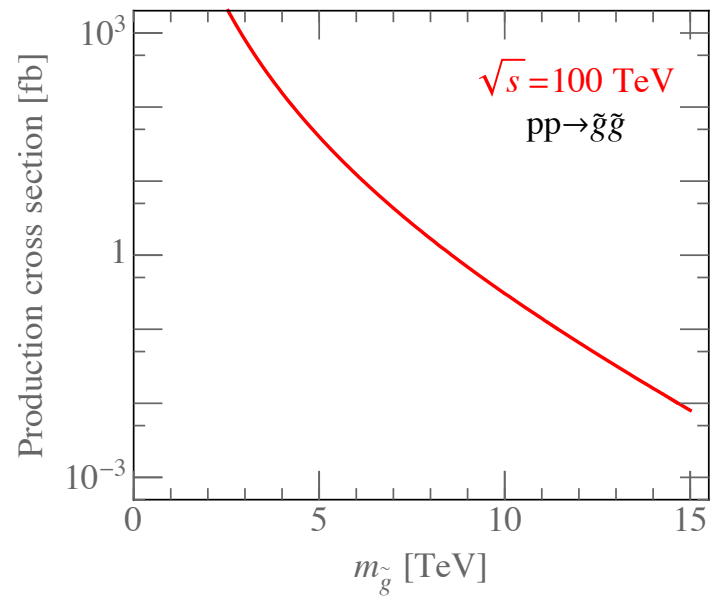

(b) $100 \mathrm{TeV}$

Figure 2. The pair-production cross sections of gluinos as functions of the gluino mass.

If the gaugino masses are induced mainly by the anomaly mediation, the gaugino mass spectrum is determined by the gauge-coupling beta functions; in particular, one finds that the LSP is the neutral wino in this case. On the other hand, if there are other contributions which are comparable to the anomaly-mediation contribution in size, the gaugino mass spectrum can deviate from the anomaly-mediation relation and, in fact, the spectrum could take any form. For example, threshold corrections by heavy Higgs bosons/higgsinos [106, 123] and some extra matter fields [124-130] give rise to such contributions. Considering this, we regard both the gluino and LSP masses as free parameters in what follows.

An interesting possibility of gaugino mass spectrum is that the LSP (say bino) is degenerate with gluino in mass with a mass splitting of $\sim 100 \mathrm{GeV}$. In this case, the annihilation cross section of the LSP in the early Universe is strongly enhanced by the coannihilation effect [131], which has significant implications for the relic abundance of the LSP. In fact, it is found that the relic abundance of the bino LSP agrees to the observed dark matter density if the mass difference between bino and gluino is $\lesssim 100 \mathrm{GeV}[30,132-$ 138]. Motivated by this observation, we will also consider such a degenerate mass spectrum in our analysis.

At hadron colliders, gluinos are pair-produced through the strong interactions, and the cross section of this process is unambiguously determined if squarks are heavy enough. We show the pair-production cross sections of gluinos as functions of the gluino mass for $\sqrt{s}=13$ and $100 \mathrm{TeV}$ in figure $2 \mathrm{a}$ and figure $2 \mathrm{~b}$, respectively. They are computed using the CTEQ6.6 PDF set [139] with the next-to-leading order QCD corrections included and soft gluon emission resummed at the next-to-leading logarithmic accuracy [140]. We will use these production cross sections in the following analyses.

\section{$4 \quad 13 \mathrm{TeV}$ LHC}

Now we study how far we may extend the reach of the prompt-decay gluino searches at the $13 \mathrm{TeV}$ LHC using a new cut based on the reconstruction of displaced vertices. We first 


\begin{tabular}{|c|c|}
\hline$m_{\tilde{g}}$ & $1000,1200, \ldots, 3200 \mathrm{GeV}$ \\
\hline \multirow{3}{*}{$m_{\tilde{\chi}_{1}^{0}}$} & $100 \mathrm{GeV}$, \\
& $m_{\tilde{g}}-(150 \mathrm{GeV}, 100 \mathrm{GeV}, 50 \mathrm{GeV}, 25 \mathrm{GeV})$ \\
\hline \multirow{2}{*}{$c \tau_{\tilde{g}}$} & $0,50,100,200,500,1000,3000 \mu \mathrm{m}$, \\
& $1 \times 10^{4}, 3 \times 10^{4}, 1 \times 10^{5}, 3 \times 10^{5}, 1 \times 10^{6} \mu \mathrm{m}$ \\
\hline
\end{tabular}

Table 1. Sample points for signal events. We generate 50000 events for each sample point.

describe our MC simulation procedure in section 4.1, which is followed by the summary of the event selection criteria used in this analysis in section 4.2. We then show the prospects of the new event-selection cut in section 4.3. Finally, in section 4.4, we discuss the possibility of measuring the lifetime of gluino in the future LHC experiments.

\subsection{MC simulation}

In the following analysis, both signal and background events are generated with MC simulations. For signal events, we use MadGraph5_aMC@NLO v2 [141] and PYTHIA v8.2 [142], and generate 50000 events for each model point categorized in terms of the gluino mass $m_{\tilde{g}}$, the LSP mass $m_{\tilde{\chi}_{1}^{0}}$, and the gluino decay length $c \tau_{\tilde{g}}$; sample points with this categorization are summarized in table $1 .{ }^{4}$ In generating the signal events, (i) the gluinos are forced to decay at the interaction point, then (ii) the decay points are spatially shifted by the flight lengths of the parent gluinos, whose probability distribution is determined from the lifetime of gluino $\tau_{\tilde{g}}$. All the trajectories of the decay products of the parent gluinos are shifted accordingly. The number of signal event samples is normalized according to the production cross section given in figure 2a. In our analysis, gluino is assumed to decay only into the first-generation quarks. In reality, gluino may also have sizable decay fractions into the other two generations of quarks, especially top quark when the stop mass is similar to or smaller than the first-generation squark masses. We will briefly discuss such cases in section 6. Moreover, we neglect the hadronization effect of gluinos as it is known that the energy and momentum of the resultant $R$-hadrons are almost the same as those of the produced gluinos, for a gluino mass of $\mathcal{O}(1) \mathrm{TeV}[1,143]$.

As for the SM background events, we consider the production processes of the electroweak gauge bosons $\left(W^{ \pm}\right.$and $Z$ ) and top-quark pairs $(t \bar{t})$. We do not consider the fully hadronic decay processes of these particles since we require a large missing energy and thus such events are expected to be efficiently eliminated. In addition, we do not take account of multi-boson production and multi-jet processes as they are found to be subdominant [144]. ${ }^{5}$

\footnotetext{
${ }^{4}$ Among these points, we do not generate events for $m_{\tilde{g}} \leq 1800 \mathrm{GeV}$ and $m_{\tilde{\chi}_{1}^{0}} \leq 600 \mathrm{GeV}$, and for $m_{\tilde{g}}=3200 \mathrm{GeV}$ and $m_{\tilde{\chi}_{1}^{0}} \neq 100 \mathrm{GeV}$.

${ }^{5}$ The multi-gauge/Higgs boson production processes may give rise to signal-like events such as four $b$ quarks plus missing transverse energy events. As it turns out, however, that the number of events for these processes is sufficiently smaller than that from the $Z$-boson plus jets processes [145], which we include in our analysis. The vertex selection cut efficiencies for these two contributions are expected to be similar, and thus we can safely drop the multi-boson production process in the following analysis.
} 


\begin{tabular}{|cc|ccccc|}
\hline & bin $\alpha$ & 0 & 1 & 2 & 3 & 4 \\
\hline$H_{T, 0}^{\max }(\alpha)$ & $(\mathrm{GeV})$ & 600 & 1200 & 2000 & 2900 & $\infty$ \\
$\sigma_{\text {matched }}(\alpha)$ & $(\mathrm{fb})$ & $8.85 \times 10^{2}$ & $5.50 \times 10^{2}$ & 66.5 & 6.6 & 0.83 \\
$N_{\text {matched }}(\alpha)$ & $\left(\times 10^{3}\right)$ & 4.6 & 53.6 & 65.3 & 198 & 28.8 \\
$\mathcal{L}_{\text {gen }}(\alpha)$ & $\left(\mathrm{ab}^{-1}\right)$ & $5.23 \times 10^{-3}$ & 0.0975 & 0.982 & 29.9 & 34.6 \\
\hline
\end{tabular}

(a) $Z \rightarrow \nu \bar{\nu}+4 j$.

\begin{tabular}{|cc|ccccc|}
\hline & bin $\alpha$ & 0 & 1 & 2 & 3 & 4 \\
\hline$H_{T, 0}^{\max }(\alpha)$ & $(\mathrm{GeV})$ & 600 & 1200 & 2000 & 2900 & $\infty$ \\
$\sigma_{\text {matched }}(\alpha)$ & $(\mathrm{fb})$ & $1.22 \times 10^{4}$ & $7.85 \times 10^{2}$ & 55.7 & 4.65 & 0.54 \\
$N_{\text {matched }}(\alpha)$ & $\left(\times 10^{3}\right)$ & 214 & 325 & 574 & 135 & 133 \\
$\mathcal{L}_{\text {gen }}(\alpha)$ & $\left(\mathrm{ab}^{-1}\right)$ & 0.0176 & 0.413 & 10.3 & 29.1 & 247 \\
\hline
\end{tabular}

(b) $Z \rightarrow \nu \bar{\nu}+0,1,2,3 j$.

\begin{tabular}{|cc|ccccc|}
\hline & $\operatorname{bin} \alpha$ & 0 & 1 & 2 & 3 & 4 \\
\hline$H_{T, 0}^{\max }(\alpha)$ & $(\mathrm{GeV})$ & 600 & 1200 & 2000 & 2900 & $\infty$ \\
$\sigma_{\text {matched }}(\alpha)$ & $(\mathrm{fb})$ & $6.28 \times 10^{4}$ & $1.04 \times 10^{3}$ & 74.4 & 6.05 & 0.69 \\
$N_{\text {matched }}(\alpha)$ & $\left(\times 10^{3}\right)$ & 280 & 223 & 212 & 207 & 205 \\
$\mathcal{L}_{\text {gen }}(\alpha)$ & $\left(\mathrm{ab}^{-1}\right)$ & $4.46 \times 10^{-3}$ & 0.214 & 2.85 & 34.1 & 300 \\
\hline
\end{tabular}

(c) $Z \rightarrow \ell \bar{\ell}+0,1,2,3 j$

Table 2. The upper edge value of $H_{T, 0}, H_{T, 0}^{\max }(\alpha)$, the leading-order matched cross section, $\sigma_{\text {matched }}$, the number of samples after matching, $N_{\text {matched }}$, and the equivalent luminosity, $\mathcal{L}_{\text {gen }}$, in each $H_{T, 0}$ bin for the $Z$-boson production process.

For the $Z$ boson production channel, the matrix elements are calculated with up to four additional partons. Due to the limitation of our available computational resources, we include only up to three and one extra partons for the $W$ and $t \bar{t}$ production processes, respectively, which are subdominant compared with the $Z$ boson production process. (We also studied these processes with one more parton. Although the number of generated events is not enough for our analysis of the gluino search, we observed that the number of SM background does not seem to be significantly changed even if we add one more parton to these samples.) The samples with different number of additional partons are merged using the five flavor MLM matching scheme with $k_{t}$ jets [146]. Here we adopt the shower- $k_{t}$ scheme and set the matching parameter QCUT $=$ XQCUT to $40 \mathrm{GeV}$ for the $Z$ and $W$ production processes and $80 \mathrm{GeV}$ for the $t \bar{t}$ production process, following ref. [147]. All generated samples are passed to PYTHIA v8.2 [142] and then DELPHES v3 [148] for the purpose of parton showering and fast detector simulation, respectively.

In new physics searches with a large integrated luminosity, it is the distribution tail of discriminating variables from the SM background processes that affects the sensitivities of these searches. A simulation of such tails in general requires a huge amount of background samples. To deal with this difficulty, we use the technique described in ref. [147]. In this 


\begin{tabular}{|cc|cccccc|}
\hline & $\operatorname{bin} \alpha$ & 0 & 1 & 2 & 3 & 4 & 5 \\
\hline$H_{T, 0}^{\max }(\alpha)$ & $(\mathrm{GeV})$ & 400 & 900 & 1600 & 2500 & 3400 & $\infty$ \\
$\sigma_{\text {matched }}(\alpha)$ & $(\mathrm{fb})$ & $5.15 \times 10^{5}$ & $3.15 \times 10^{4}$ & $2.10 \times 10^{3}$ & $1.74 \times 10^{2}$ & 14.2 & 1.92 \\
$N_{\text {matched }}(\alpha)$ & $\left(\times 10^{3}\right)$ & 286 & 233 & 2180 & 1680 & 414 & 206 \\
$\mathcal{L}_{\text {gen }}(\alpha)$ & $\left(\mathrm{ab}^{-1}\right)$ & $5.5 \times 10^{-4}$ & $7.41 \times 10^{-3}$ & 1.04 & 9.64 & 29.2 & 107 \\
\hline
\end{tabular}

Table 3. Same as in table 2, but for the $W$ production process.

\begin{tabular}{|cc|cccccc|}
\hline & $\operatorname{bin} \alpha$ & 0 & 1 & 2 & 3 & 4 & 5 \\
\hline$H_{T, 0}^{\max }(\alpha)$ & $(\mathrm{GeV})$ & 600 & 1100 & 1700 & 2400 & 3200 & $\infty$ \\
$\sigma_{\text {matched }}(\alpha)$ & $(\mathrm{fb})$ & $7.43 \times 10^{4}$ & $5.64 \times 10^{3}$ & $4.26 \times 10^{2}$ & 36.8 & 3.43 & 0.34 \\
$N_{\text {matched }}(\alpha)$ & $\left(\times 10^{3}\right)$ & 154 & 1220 & 1280 & 177 & 182 & 186 \\
$\mathcal{L}_{\text {gen }}(\alpha)$ & $\left(\mathrm{ab}^{-1}\right)$ & $2.08 \times 10^{-3}$ & 0.216 & 2.99 & 4.81 & 53.0 & 552 \\
\hline
\end{tabular}

(a) $t \bar{t} \rightarrow$ (semi-leptonic) $+0,1 j$.

\begin{tabular}{|cc|cccc|}
\hline & $\operatorname{bin} \alpha$ & 0 & 1 & 2 & 3 \\
\hline$H_{T, 0}^{\max }(\alpha)$ & $(\mathrm{GeV})$ & 800 & 1400 & 2100 & $\infty$ \\
$\sigma_{\text {matched }}(\alpha)$ & $(\mathrm{fb})$ & 418 & 38.9 & 3.74 & 0.34 \\
$N_{\text {matched }}(\alpha)$ & $\left(\times 10^{3}\right)$ & 41 & 48 & 97 & 49 \\
$\mathcal{L}_{\text {gen }}(\alpha)$ & $\left(\mathrm{ab}^{-1}\right)$ & 0.0980 & 1.22 & 26.0 & 146 \\
\hline
\end{tabular}

(b) $t \bar{t} \rightarrow$ (leptonic) $+0,1 j$.

Table 4. Same as in table 2 , but for the $t \bar{t}$ production process.

method, the generator-level phase space is divided into several regions, and $\mathrm{MC}$ samples are separately generated and matched for each region. The generated samples are then combined with each other after multiplied by some weight factor. In our analysis, we divide the generator-level phase space into bins numbered by $\alpha=0,1,2, \ldots$, in terms of the scalar sum of $p_{\mathrm{T}}$ of partons in each event:

$$
H_{T, 0} \equiv \sum_{\text {partons }} p_{\mathrm{T}}
$$

We show the values of the upper edge of each bin $\alpha$, denoted by $H_{T, 0}^{\max }(\alpha)$, for the $Z, W$, and $t \bar{t}$ production processes in tables 2,3 , and 4 , respectively. Here, the width of the bin $\alpha+1$ is determined such that the corresponding cross section is $\simeq 1 / 10$ of that of the bin $\alpha .{ }^{6}$ We also give the leading-order matched cross section $\sigma_{\text {matched }}$, the number of samples after matching $N_{\text {matched }}$, and the equivalent luminosity $\mathcal{L}_{\text {gen }}=N_{\text {matched }} / \sigma_{\text {matched }}$ in these tables. ${ }^{7}$ When generating these samples, we impose a generator-level cut of $\left|\sum_{\text {all } \nu} \boldsymbol{p}_{\mathrm{T}}\right|>$ $200 \mathrm{GeV}$ for $Z \rightarrow \nu \bar{\nu}$ and $\left|\sum_{\text {all } l, \nu} \boldsymbol{p}_{\mathrm{T}}\right|>100 \mathrm{GeV}$ for the other processes. We then define

\footnotetext{
${ }^{6}$ For $Z$ production processes, the widths of the bins are taken universal; the width of the bin $\alpha+1$ is determined such that the corresponding cross section becomes $\sim 1 / 10$ of that of the bin $\alpha$ for $Z \rightarrow$ $\nu \bar{\nu}+0,1,2,3 j$.

${ }^{7}$ We do not include $K$-factors in our analysis.
} 


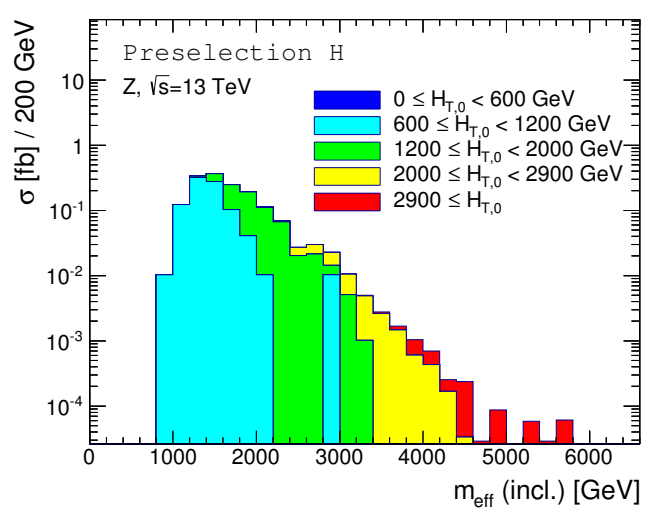

(a) $Z$ boson production

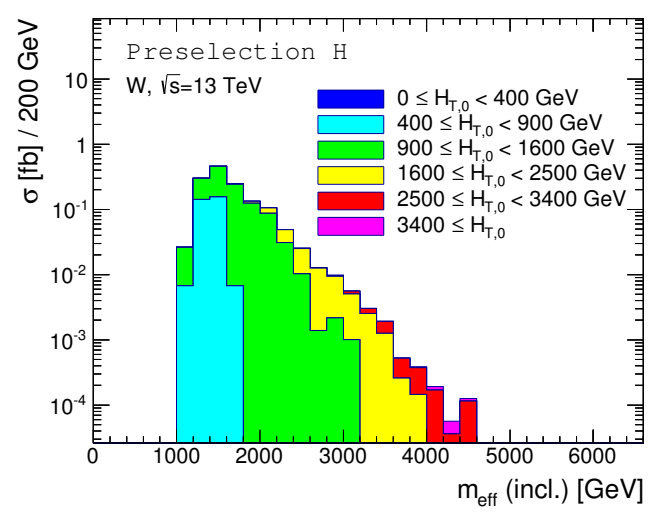

(b) $W$ boson production

Figure 3. Distributions of $m_{\text {eff }}($ incl.) with preselection-H required. Contributions from different $H_{T, 0}$ bins are filled with different colors.

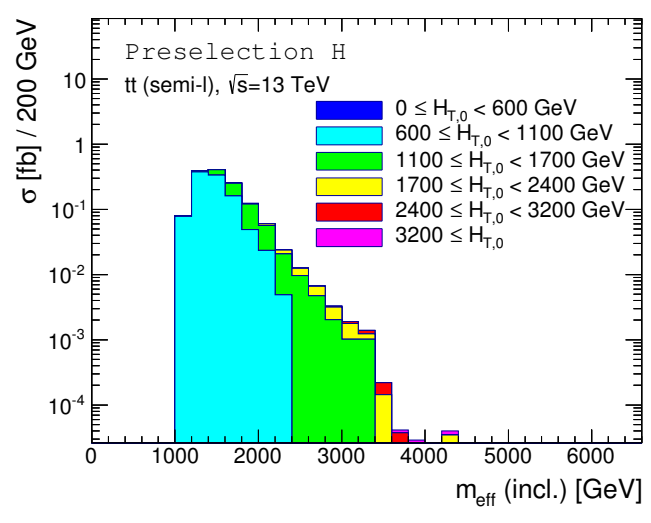

(a) Semi-leptonic $t \bar{t}$ decay

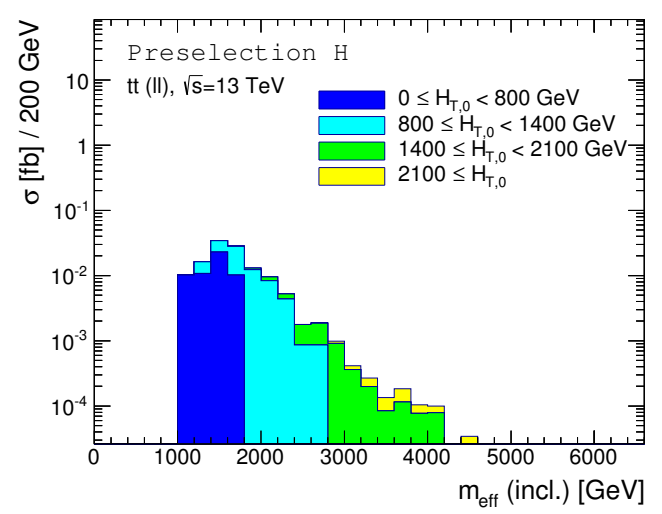

(b) Leptonic $t \bar{t}$ decay

Figure 4. Same as in figure 3, but for the $t \bar{t}$ production process.

the weight $w_{\alpha}$ for the samples in the bin $\alpha$ by

$$
w_{\alpha} \equiv \frac{\sigma_{\text {matched }}(\alpha)}{N_{\text {matched }}(\alpha)},
$$

and all samples in the bins are added by using these weights.

By using the samples obtained above, we generate the distribution of $m_{\mathrm{eff}}$ (incl.) for each process, where $m_{\text {eff }}($ incl. $)$ is defined as the scalar sum of the missing transverse energy $E_{\mathrm{T}}^{\text {(miss) }}$ and the transverse momenta of all jets with $p_{\mathrm{T}}>50 \mathrm{GeV}$; the results are given in figures 3 and 4 . These distributions are obtained for events passing a selection requirement Preselection- $\mathrm{H}$, whose definition is found in table 5 in the next section. We will use $m_{\text {eff }}($ incl.) to define signal regions in the following analyses. From these figures, we find that there is a correlation between $H_{T, 0}$ and $m_{\text {eff }}$ (incl.). In addition, we find a sizable number of events in the tails of the distributions, which smoothly spread out from the bulk of the distributions. These observations indicate that the division of the generator-level 
phase space in terms of $H_{T, 0}$ offers an adequate way to estimate the number of events in signal regions which are defined by the values of $m_{\mathrm{eff}}$ (incl.).

For the definition of the objects such as charged tracks, jets, and charged leptons, we basically follow ref. [144]. We require $p_{\mathrm{T}}>1 \mathrm{GeV}$ and $|\eta|<2.5$ for the reconstruction of a charged track, and reject it if the production point of the particle associated with the charged track is outside the innermost pixel layer, which is located at $\left|\boldsymbol{r}_{\mathrm{T}}\right|=33.25 \mathrm{~mm}$. These requirements are intended to remove tracks with poor measurement quality. Jets are clustered using Fast Jet v3.1 [149] with a jet radius parameter of 0.4 and required to satisfy $p_{\mathrm{T}}>20 \mathrm{GeV}$ and $|\eta|<2.8$. For leptons, we require $p_{\mathrm{T}}>7 \mathrm{GeV}$, and $|\eta|<2.47$ (2.7) for electrons (muons).

As we will mention in the next section, we require neither electrons nor muons be reconstructed in our analysis. In this case, the SM background processes listed above can contribute to the signal regions only if all of the leptons in the processes fail to be reconstructed. To take account of this, we introduce the reconstruction probability of an electron (muon), $p_{\text {reco. }}^{e(\mu)}\left(p_{\mathrm{T}}, \eta\right)$, which we take from DELPHES v3 [148]. We then multiply the weight $w_{\alpha}$ in eq. (4.2) by a factor of $\prod_{\text {all } e_{i}}\left(1-p_{\text {reco. }}^{e_{i}}\right) \times \prod_{\text {all } \mu_{i}}\left(1-p_{\text {reco. }}^{\mu_{i}}\right)$ when we require the absence of electrons and muons.

To validate our MC simulations, we compute the expected number of background events as well as the expected 95\% confidence level (CL) exclusion limit on gluino mass for an integrated luminosity of $36.1 \mathrm{fb}^{-1}$ at the $13 \mathrm{TeV}$ LHC. We use the kinematical selection category called Meff-4j-3000 given in ref. [144]. ${ }^{8}$ We then find the expected number of background events to be 1.4 and the exclusion limit on the gluino mass to be $1960 \mathrm{GeV}$. Comparing these results with the ones reported by the ATLAS collaboration [144] -2.0 and $2030 \mathrm{GeV}$, respectively — we can safely conclude that our MC simulation satisfactorily reproduces the ATLAS results.

\subsection{Event selection criteria}

In our analysis, we impose a new event-selection cut using the information of displaced vertices in addition to the ordinary selection criteria based on kinematics. We further try to optimize the kinematic-based cuts as the new selection cut is expected to reduce the SM background efficiently. To that end, we first divide the kinematic-based selection criteria into two classes; $m_{\text {eff }}$ (incl.), which is to be varied to optimize the event selection, and the other criteria adopted in ref. [144], which we call the preselection. Moreover, we divide the preselection into three classes, preselection-L, -M, and $-\mathrm{H}$, and use one of them for each sample point so that the sensitivity is maximized. We summarize the preselection criteria in table 5 . Here, $p_{\mathrm{T}}\left(j_{i}\right)$ denotes the transverse momentum of $i$-th jet, $\Delta \phi$ is the azimuthal angle between the jet and the missing energy, and $m_{\text {eff }}(4)$ is the scalar sum of $E_{\mathrm{T}}^{\text {(miss) }}$ and the transverse momenta of the leading 4-jets. The requirements on $\Delta \phi$ and $E_{\mathrm{T}}^{\text {(miss) }} / m_{\text {eff }}(4)$ were imposed to reduce the contributions from QCD multi-jet processes in ref. [144] —although such multi-jet processes are not included in the present analysis,

\footnotetext{
${ }^{8}$ When we require a large $m_{\text {eff }}$ (incl.) as in Meff-4j-3000, the diboson production channel may become comparable to the $W$ and $t \bar{t}$ production channels [144], though this is still subdominant compared with the $Z$-boson production process.
} 


\begin{tabular}{|c|c|c|c|}
\hline Requirement & $\mathrm{L}$ & M & $\mathrm{H}$ \\
\hline Number of jets with $p_{\mathrm{T}}>50 \mathrm{GeV}$ & \multicolumn{3}{|c|}{$\geq 4$} \\
\hline$E_{\mathrm{T}}^{(\text {miss })}[\mathrm{GeV}]$ & \multicolumn{3}{|c|}{$>250$} \\
\hline$p_{\mathrm{T}}\left(j_{1}\right)[\mathrm{GeV}]$ & \multicolumn{3}{|c|}{$>200$} \\
\hline$p_{\mathrm{T}}\left(j_{4}\right)[\mathrm{GeV}]$ & $>100$ & $>100$ & $>150$ \\
\hline$\left|\eta\left(j_{i=1,2,3,4}\right)\right|$ & $<1.2$ & $<2.0$ & $<2.0$ \\
\hline$\Delta \phi\left(j_{p_{\mathrm{T}}>50 \mathrm{GeV}}, E_{\mathrm{T}}^{(\text {miss })}\right)_{\min }$ & \multicolumn{3}{|c|}{$>0.4$} \\
\hline$E_{\mathrm{T}}^{(\text {miss })} / m_{\mathrm{eff}}(4)$ & $>0.3$ & $>0.25$ & $>0.2$ \\
\hline Aplanarity & \multicolumn{3}{|c|}{$>0.04$} \\
\hline
\end{tabular}

Table 5. Definition of preselection-L, M, H. Besides these criteria, we require that neither electrons nor muons be reconstructed.

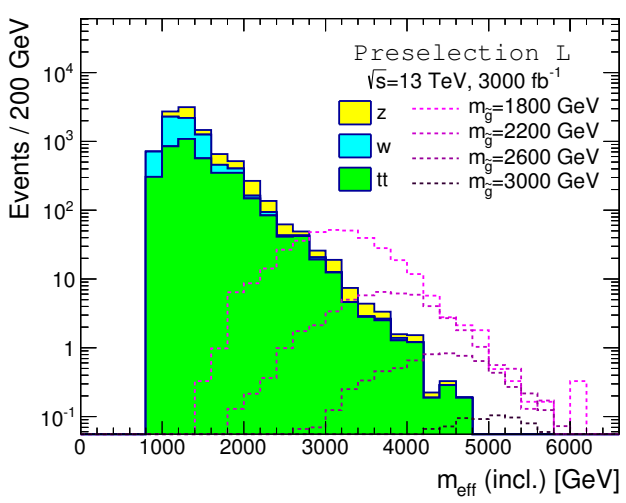

(a) Preselection-L

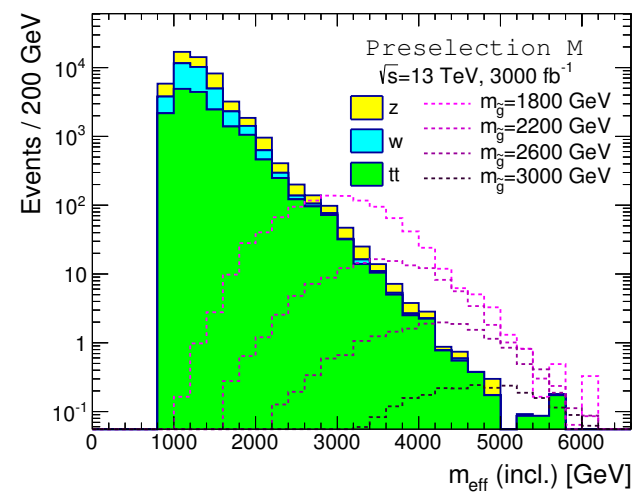

(b) Preselection-M

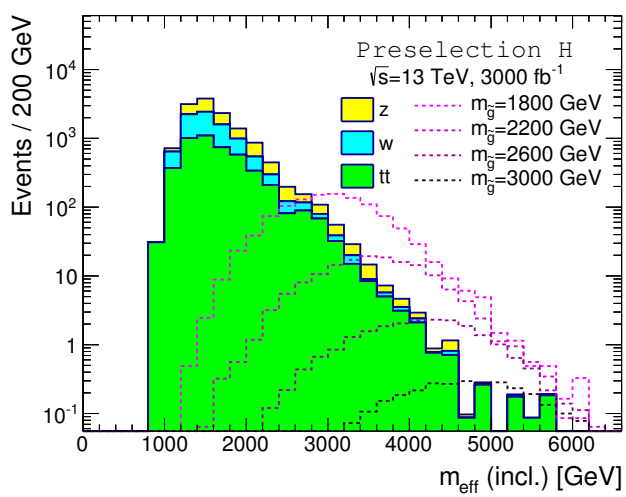

(c) Preselection-H

Figure 5. Distributions of $m_{\mathrm{eff}}($ incl.) for the SM background and gluino events. We set the LSP mass to be $100 \mathrm{GeV}$. 
we still impose these requirements since they are also able to reject background events from the semi-leptonically decaying $t$ and $\bar{t}$ efficiently. The definition of aplanarity can be found in refs. [150, 151]; if a distribution of jets is highly directional, then the value of aplanarity gets close to zero, while if it is completely isotropic then this is equal to $1 / 2$. In addition to these kinematical selection criteria, we require that neither electrons nor muons be reconstructed. In figure 5 , we give the distributions of $m_{\text {eff }}$ (incl.) for the SM background and gluino events with each preselection imposed. Here, we set the LSP mass to be $100 \mathrm{GeV}$. These figures show that we may effectively select gluino events while eliminating the SM background if we require a large $m_{\mathrm{eff}}$ (incl.). The size of this cut will be chosen so that the sensitivity is maximized, as we see in the subsequent section. We further note in passing that because of the requirement of a large $m_{\text {eff }}($ incl. $)$ the event-selection efficiency has small sensitivity to the choice of the $E_{\mathrm{T}}^{(\mathrm{miss})}$ threshold - thus, we expect that our result here is less affected even if the $E_{\mathrm{T}}^{(\text {miss })}$ trigger requirement is tightened in the future.

Now we discuss a selection criterion based on the reconstruction of displaced vertices. As described in section 2, we reconstruct vertices using charged tracks associated with the decay product. The resolutions of impact parameters, which determine the resolution of vertex reconstruction, are obtained by fitting eq. (2.2) onto the experimental results given by the ATLAS collaboration [88, 89]. As we mentioned in section 4.1, only the tracks with $p_{\mathrm{T}}>1 \mathrm{GeV}$ are used in this analysis. Performing the fit in this range, we determine the parameters in eq. $(2.2)$ as $\sigma_{d_{0}}(\infty)=23 \mu \mathrm{m}, \sigma_{z_{0} \sin \theta}(\infty)=78 \mu \mathrm{m}, p_{d_{0}}=3.1 \mathrm{GeV}$, and $p_{z_{0} \sin \theta}=1.6 \mathrm{GeV}$.

As we discussed in section 2, we use four high- $p_{\mathrm{T}}$ jets to reconstruct displaced vertices generated in gluino-decay events. For this purpose, we basically choose the four-highest $p_{\mathrm{T}}$ jets. We further require that the tracks in these jets satisfy $d_{0}<10 \mathrm{~mm}$ and $\left|z_{0}\right|<320 \mathrm{~mm}$ in order to focus on decays that occur inside the inner detector. However, if one of these four jets contains no track which satisfies the above conditions, then we add the fifthhighest $p_{\mathrm{T}}$ jet to the vertex reconstruction analysis. If the number of jets which contain tracks satisfying the criteria is smaller than four among these five jets, then we conclude that the reconstruction of vertices fails in such an event.

As our main focus is on displaced vertices located inside the innermost pixel layer, vertices generated by hadronic interactions in the detector materials rarely contribute to the background. To assure this, we reject events in which a vertex is reconstructed inside the detector materials: i.e., $22 \mathrm{~mm} \leq\left|\left(\boldsymbol{r}_{\mathrm{DV} 1,2}\right)_{\mathrm{T}}\right| \leq 25 \mathrm{~mm}, 29 \mathrm{~mm} \leq\left|\left(\boldsymbol{r}_{\mathrm{DV} 1,2}\right)_{\mathrm{T}}\right| \leq$ $38 \mathrm{~mm}, 46 \mathrm{~mm} \leq\left|\left(\boldsymbol{r}_{\mathrm{DV} 1,2}\right)_{\mathrm{T}}\right| \leq 73 \mathrm{~mm}, 84 \mathrm{~mm} \leq\left|\left(\boldsymbol{r}_{\mathrm{DV} 1,2}\right)_{\mathrm{T}}\right| \leq 111 \mathrm{~mm}$, or $\left|\left(\boldsymbol{r}_{\mathrm{DV} 1,2}\right)_{\mathrm{T}}\right| \geq$ $120 \mathrm{~mm}[90,152-154]$. In the absence of this possibility, the displaced vertices from the SM processes are mainly caused by the misinterpretation of non-displaced vertices due to the limited resolution of track impact parameters, which we take into account in a manner discussed in section 2 .

In the metastable gluino production processes under consideration, we do not expect that particles with hard momenta are generated at the primary interaction point (except those from initial state radiation), as the metastable gluinos decay after they flew away 


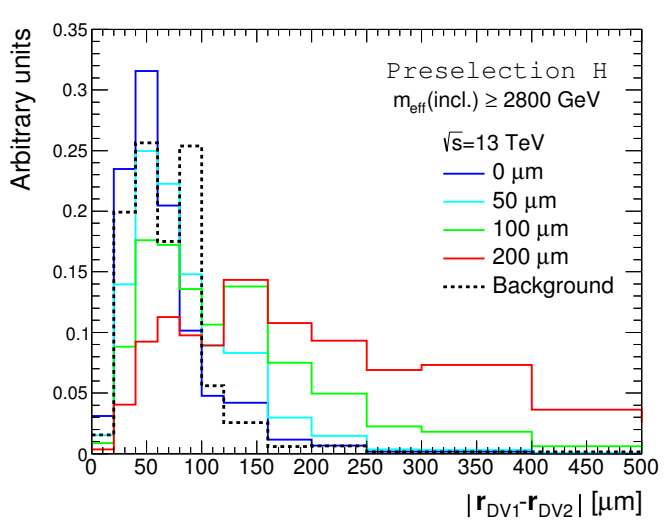

(a) $\left|\boldsymbol{r}_{\mathrm{DV} 1}-\boldsymbol{r}_{\mathrm{DV} 2}\right|$ distribution

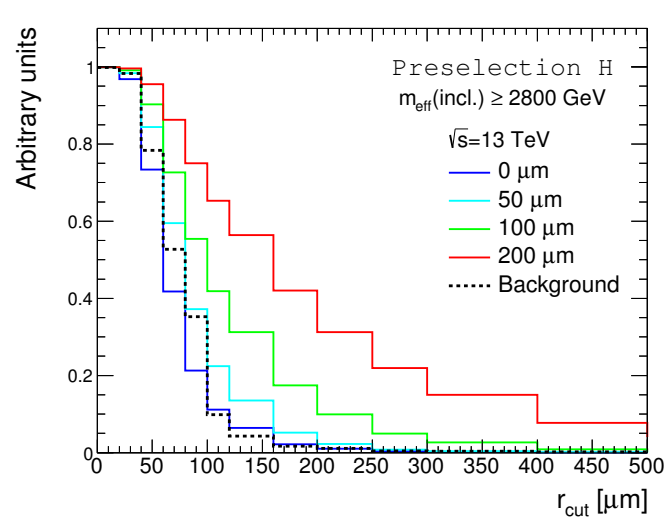

(b) Efficiency

Figure 6. The distributions of $\left|\boldsymbol{r}_{\mathrm{DV} 1}-\boldsymbol{r}_{\mathrm{DV} 2}\right|$ and the fractions to pass the selection cut $\mid \boldsymbol{r}_{\mathrm{DV} 1}-$ $\boldsymbol{r}_{\mathrm{DV} 2} \mid>r_{\text {cut }}$ for a gluino with $m_{\tilde{g}}=3 \mathrm{TeV}$ and different values of $c \tau_{\tilde{g}}$, shown in the solid lines. The distributions for the SM background events are also shown in the dotted lines. We have imposed preselection-H and $m_{\text {eff }}$ (incl.) $>2800 \mathrm{GeV}$.

from the primary interaction point. For this reason, we do not try to determine the position of the primary interaction point in each event. ${ }^{9}$ Instead, we use the distance between the two reconstructed vertices as a discriminator. As for the definition of the distance, we try the following three candidates and adopt the one which yields the best sensitivity for each sample point: $\left|\boldsymbol{r}_{\mathrm{DV} 1}-\boldsymbol{r}_{\mathrm{DV} 2}\right|,\left|\left(\boldsymbol{r}_{\mathrm{DV} 1}-\boldsymbol{r}_{\mathrm{DV} 2}\right)_{\mathrm{T}}\right|$, and $\left|\left(\boldsymbol{r}_{\mathrm{DV} 1}-\boldsymbol{r}_{\mathrm{DV} 2}\right)_{z}\right|$, where $\boldsymbol{r}_{\mathrm{DV} 1,2}$ are the position vectors of the displaced vertices defined in section 2 , and $\mathrm{T}$ and $z$ stand for the transverse and $z$ directions, respectively.

In figure 6a, we show the $\left|\boldsymbol{r}_{\mathrm{DV} 1}-\boldsymbol{r}_{\mathrm{DV} 2}\right|$ distribution of signal events for a gluino with $m_{\tilde{g}}=3 \mathrm{TeV}$ and different values of the decay distance $c \tau_{\tilde{g}}$ in the solid lines. The distribution for the SM background events is also shown in the dotted line. Figure $6 \mathrm{~b}$ shows a fraction of events passing a selection cut of $\left|\boldsymbol{r}_{\mathrm{DV} 1}-\boldsymbol{r}_{\mathrm{DV} 2}\right|>r_{\text {cut }}$ as a function of $r_{\text {cut }}$. For these simulations, we have imposed preselection-H and $m_{\text {eff }}$ (incl.) $>2800 \mathrm{GeV}$. Notice that the distribution of the SM background deviates from the signal distribution with $c \tau_{\tilde{g}}=0$. This is because the flavor content of jets in the SM background sample is different from that in the signal event sample. In the signal events, gluinos are forced to decay into only the first-generation quarks as we mentioned above. In the SM background events, on the other hand, jets may also be induced by heavy flavor quarks, which then contain metastable hadrons; for example, the typical decay length of $B$ mesons is about $400 \mu \mathrm{m}$ and these mesons can fly over a few $\mathrm{mm}$ when they are highly boosted. Decay products of such metastable hadrons may form a secondary vertex and deteriorate resolution of the vertex reconstruction position. We however note that our vertex reconstruction method is actually robust against the presence of metastable hadrons. A jet originating from a heavy

\footnotetext{
${ }^{9}$ We however note that it may be possible to reconstruct the primary vertex by using the initial state radiation and/or soft products generated by remnants of the $p p$ collision. Implications of this possibility will be discussed in section 6 .
} 


\begin{tabular}{|c|c|}
\hline Selection & Requirements \\
\hline $\begin{array}{l}\text { Preselection } \\
\text { Lepton veto }\end{array}$ & $\begin{array}{l}\quad \in(\text { preslection-L, } \mathrm{M}, \mathrm{H}) \\
\text { No reconstructed electrons and muons }\end{array}$ \\
\hline Material veto & $\begin{array}{l}\text { No vertices reconstructed in material regions: } \\
\text { i.e. (in units of } \mathrm{mm}) \\
\left|\left(\boldsymbol{r}_{\mathrm{DV}}\right)_{\mathrm{T}}\right| \notin(22,25),(29,38),(46,73),(84,111),(120, \infty)\end{array}$ \\
\hline$m_{\text {eff }}$ (incl.) cut & $\begin{array}{l}\text { Require } m_{\text {eff }}(\text { incl. })>\left(m_{\text {effcut }}\right)_{\text {optimal }} \\
\text { over } \\
m_{\text {effcut }} \in\left(1000 \mathrm{GeV}, 10^{4} \mathrm{GeV}\right)\end{array}$ \\
\hline$\Delta r_{\text {DV }}$ cut & $\begin{array}{c}\text { Require } \Delta r_{\mathrm{DV}}>\left(r_{\text {cut }}\right)_{\text {optimal }} \\
\text { over } \\
\Delta r_{\mathrm{DV}} \in\left(\left|\Delta \boldsymbol{r}_{\mathrm{DV}}\right|,\left|\left(\Delta \boldsymbol{r}_{\mathrm{DV}}\right)_{\mathrm{T}}\right|,\left|\left(\Delta \boldsymbol{r}_{\mathrm{DV}}\right)_{\mathrm{z}}\right|\right) \\
r_{\text {cut }} \in\left(0,2 \times 10^{5} \mu \mathrm{m}\right)\end{array}$ \\
\hline
\end{tabular}

Table 6. Summary of event selection criteria.

flavor quark contains not only metastable hadrons such as $B$ mesons but also other many hadrons emitted during hadronization. Since our vertex reconstruction algorithm chooses as the vertex position a point at which tracks are most densely concentrated, it is less likely to identify a secondary vertex caused by a metastable hadron as the reconstructed vertex. For the same reason, our vertex reconstruction method is less affected by pile-up events as well, especially if we reconstruct vertices using only tracks in high- $p_{\mathrm{T}}$ jets as we do in our analysis. In fact, the ATLAS collaboration found in ref. [156] that the reconstruction efficiencies of vertices associated with hard-scattering $t \bar{t}$ events are not affected by pile-up, which supports our expectation. The effect of pile-up events on kinematical selection cuts is also expected to be small [145]. Considering these, we do not include the pile-up effects in our analysis.

The plots in figure 6 show that if we set $r_{\text {cut }}$ to be $\gtrsim 100 \mu \mathrm{m}$, then a significant fraction of the SM background fails to pass the selection cut while a sizable number of signal events for $c \tau_{\tilde{g}} \gtrsim 100 \mu \mathrm{m}$ still remain after the selection cut. This observation indicates that this cut may be useful to probe a gluino with a decay length of $c \tau_{\tilde{g}} \gtrsim 100 \mu \mathrm{m}$, which we demonstrate in the subsequent section.

\subsection{Prospects}

Let us summarize the event selection criteria we use in the following analysis. After we apply one of the preselections, preselection-L, $-\mathrm{M},-\mathrm{H}$, we further require

$$
m_{\text {eff }}(\text { incl. })>\left(m_{\text {effcut }}\right)_{\text {optimal }} \text { and } \Delta r_{\text {DV }}>\left(r_{\text {cut }}\right)_{\text {optimal }}
$$

where $\Delta r_{\mathrm{DV}}$ is one of the discriminators proposed in the previous section, i.e., $\Delta r_{\mathrm{DV}}=$ $\left|\Delta \boldsymbol{r}_{\mathrm{DV}}\right|,\left|\left(\Delta \boldsymbol{r}_{\mathrm{DV}}\right)_{\mathrm{T}}\right|$, or $\left|\left(\Delta \boldsymbol{r}_{\mathrm{DV}}\right)_{z}\right|$, where $\Delta \boldsymbol{r}_{\mathrm{DV}} \equiv \boldsymbol{r}_{\mathrm{DV} 1}-\boldsymbol{r}_{\mathrm{DV} 2}$. We adopt the one which leads to the best sensitivity for a given sample point. We vary the cut parameters $m_{\text {effcut }}$ and $r_{\text {cut }}$ in eq. (4.3) from $1000 \mathrm{GeV}$ to $10^{4} \mathrm{GeV}$ and from $0 \mu \mathrm{m}$ to $2 \times 10^{5} \mu \mathrm{m}$, respectively, 
and employ the values which maximize the performance. These event selection criteria are listed in table 6 .

Now we study the performance of the new selection cut based on the reconstruction of displaced vertices. To that end, we evaluate the discovery reach and exclusion limit for metastable gluino searches and compare them with the existing results from the prompt decay searches. For exclusion limits, we compute the expected $95 \%$ confidence level (CL) limits on the gluino mass using the $C L_{s}$ prescription [155, 157]. For the discovery reach, we compute the expected significance of discovery $Z_{0}[158]$ :

$$
Z_{0}=\sqrt{2\{(S+B) \log (1+S / B)-S\}}
$$

where $S$ and $B$ are the expected numbers of signal and background events, respectively. We then require both $Z_{0}$ and $S$ to be larger than 5 for the discovery.

In figure 7 , we show optimal cut values $\left(\left(m_{\text {effcut }}\right)_{\text {optimal }},\left(r_{\text {cut }}\right)_{\text {optimal }}\right)$, the preselection (L, M, or $\mathrm{H})$, and the discriminator $\Delta r_{\mathrm{DV}}(\mathrm{R}, \mathrm{T}$, or $\mathrm{Z})$ for each sample point with various $c \tau_{\tilde{g}}$, with an integrated luminosity of $3000 \mathrm{fb}^{-1}$. Here, for the discriminator $\Delta r_{\mathrm{DV}}, \mathrm{R}$, $\mathrm{T}$, and $\mathrm{Z}$ represent $\left|\Delta \boldsymbol{r}_{\mathrm{DV}}\right|,\left|\left(\Delta \boldsymbol{r}_{\mathrm{DV}}\right)_{\mathrm{T}}\right|$, and $\left|\left(\Delta \boldsymbol{r}_{\mathrm{DV}}\right)_{z}\right|$, respectively. They are obtained so that the expected significance $Z_{0}$ is maximized. The expected $5 \sigma$ discovery reaches for gluinos are also shown in the dashed lines, which we discuss in more detail below. It is found that the new selection cut allows us to relax the kinematic selection cut on $m_{\text {eff }}$ (incl.) considerably, especially for gluinos with a long lifetime. As for the optimization of $\left(r_{\text {cut }}\right)_{\text {optimal }},\left(r_{\text {cut }}\right)_{\text {optimal }} \simeq c \tau_{\tilde{g}}$ tends to be favored for gluinos with a sub-millimeter decay length.

In tables 7 and 8 , we present the expected number of background and signal events for an integrated luminosity of $\mathcal{L}=3000 \mathrm{fb}^{-1} \cdot{ }^{10}$ In table 7 (8), we consider the case of a light (heavy) LSP with $m_{\tilde{g}}=2800(2400) \mathrm{GeV}$ and $m_{\tilde{\chi}_{1}^{0}}=100$ (1440) GeV, where preselection-H (-M) is used for the event preselection. We see that in both cases the new selection cut efficiently removes the SM background while maintaining a sizable amount of signal events for metastable gluinos. To see this more clearly, in figure 8 , we show the distributions of $m_{\text {eff }}$ (incl.) for the SM background and signal events with different values of $c \tau_{\tilde{g}}$, with preselection-H and the vetoes in table 6 imposed. The masses of gluino and the LSP are set to be $2600 \mathrm{GeV}$ and $100 \mathrm{GeV}$, respectively. As seen in these plots, the new selection cut considerably reduces the SM background especially if we require a large separation between the reconstructed displaced vertices, which allows us to relax the cut on $m_{\text {eff }}$ (incl.) to keep a large number of signal events.

Now we show in figure 9 the expected limit on the gluino mass as a function of $c \tau_{\tilde{g}}$ based on the currently available luminosity of $36.1 \mathrm{fb}^{-1}$ at the $13 \mathrm{TeV}$ LHC (red solid line). Here the mass of LSP is set to be $100 \mathrm{GeV}$. The improvement in reach because

\footnotetext{
${ }^{10}$ We also show the statistical uncertainties of MC simulation, which we estimate as$$
\Delta N=\mathcal{L} \times \sqrt{\sum w_{\text {sample }}^{2}},
$$

where $\mathcal{L}$ is an integrated luminosity, $w_{\text {sample }}$ is the MC sample weight given by eq. (4.2), and the summation is taken over all MC samples which have passed the selection cut.
} 


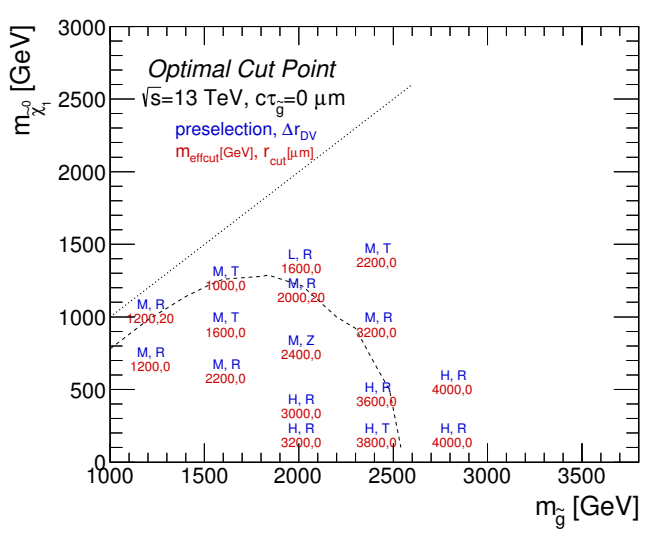

(a) $c \tau_{\tilde{g}}=0 \mu \mathrm{m}$

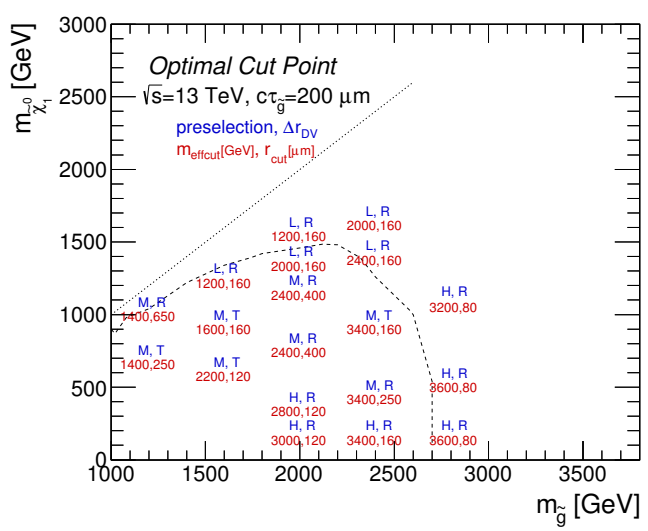

(c) $c \tau_{\tilde{g}}=200 \mu \mathrm{m}$

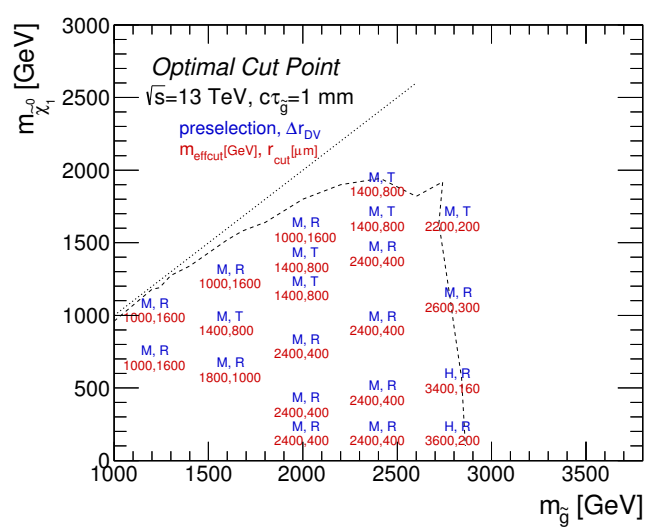

(e) $c \tau_{\tilde{g}}=1 \mathrm{~mm}$

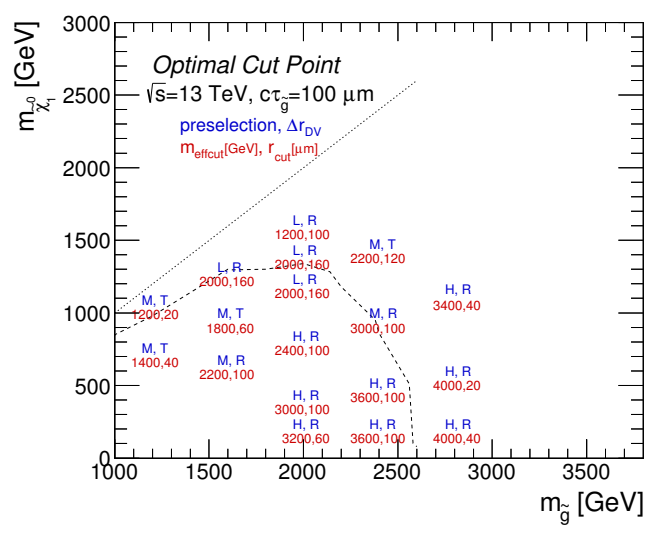

(b) $c \tau_{\tilde{g}}=100 \mu \mathrm{m}$

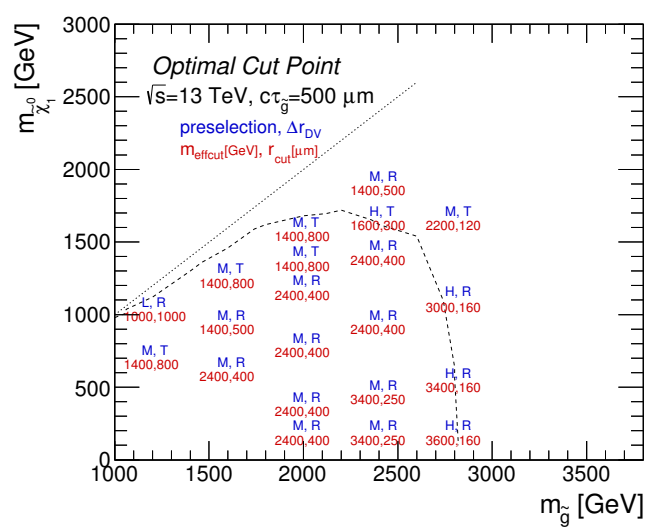

(d) $c \tau_{\tilde{g}}=500 \mu \mathrm{m}$

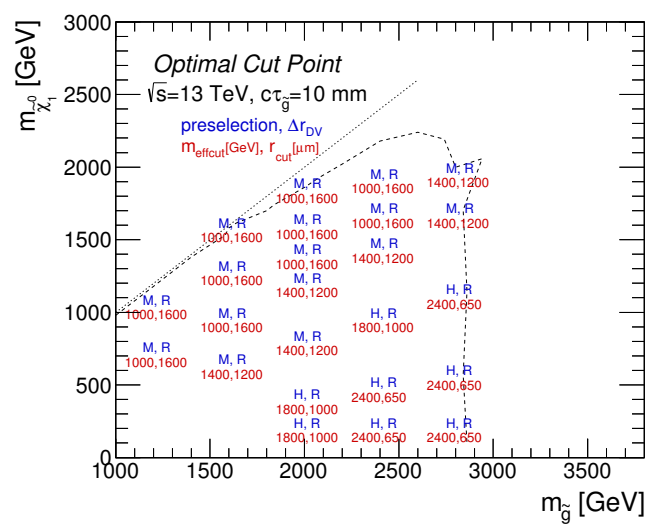

(f) $c \tau_{\tilde{g}}=10 \mathrm{~mm}$

Figure 7. Optimal cut values $\left(\left(m_{\text {effcut }}\right)_{\text {optimal }},\left(r_{\text {cut }}\right)_{\text {optimal }}\right)$, the preselection $(\mathrm{L}, \mathrm{M}, \mathrm{H})$, and the discriminator $\Delta r_{\mathrm{DV}}(\mathrm{R}, \mathrm{T}, \mathrm{Z})$ for each sample point with various $c \tau_{\tilde{g}}$, with an integrated luminosity of $3000 \mathrm{fb}^{-1}$. The expected $5 \sigma$ discovery reaches for gluinos are also shown in the dashed lines. 


\begin{tabular}{|l|ccc|c|}
\hline & $Z$ & $W$ & $t \bar{t}$ & total \\
\hline preselection-H $\left(\times 10^{3}\right)$ & $4.7 \pm 0.3$ & $4.1 \pm 0.6$ & $4.5 \pm 0.2$ & $13.3 \pm 0.7$ \\
$m_{\text {eff }}$ (incl.) $>3600 \mathrm{GeV}$ & $12.5 \pm 1$ & $4.1 \pm 0.7$ & $1.6 \pm 0.4$ & $18.2 \pm 1$ \\
\hline$\left|\boldsymbol{r}_{\mathrm{DV} 1}-\boldsymbol{r}_{\mathrm{DV} 2}\right|>80 \mu \mathrm{m}$ & $2.1 \pm 0.4$ & $0.8 \pm 0.4$ & $0.2 \pm 0.1$ & $3.2 \pm 0.6$ \\
$\left|\boldsymbol{r}_{\mathrm{DV} 1}-\boldsymbol{r}_{\mathrm{DV} 2}\right|>160 \mu \mathrm{m}$ & $0.1 \pm 0.1$ & $<0.1$ & $<0.1$ & $0.1 \pm 0.1$ \\
$\left|\boldsymbol{r}_{\mathrm{DV} 1}-\boldsymbol{r}_{\mathrm{DV} 2}\right|>200 \mu \mathrm{m}$ & $0.1 \pm 0.1$ & $<0.1$ & $<0.1$ & $0.1 \pm 0.1$ \\
\hline
\end{tabular}

(a) The expected number of background events.

\begin{tabular}{|l|cccc|}
\hline & $c \tau_{\tilde{g}}=0$ & $c \tau_{\tilde{g}}=200 \mu \mathrm{m}$ & $c \tau_{\tilde{g}}=500 \mu \mathrm{m}$ & $c \tau_{\tilde{g}}=1 \mathrm{~mm}$ \\
\hline preselection-H & \multicolumn{4}{|c|}{$8.2 \pm 0.1$} \\
$m_{\text {eff }}$ (incl.) $>3600 \mathrm{GeV}$ & \multicolumn{4}{|c|}{$6.9 \pm 0.1$} \\
\hline$\left|\boldsymbol{r}_{\mathrm{DV} 1}-\boldsymbol{r}_{\mathrm{DV} 2}\right|>80 \mu \mathrm{m}$ & $1.6 \pm 0.04$ & $5.3 \pm 0.1$ & $6.3 \pm 0.1$ & $6.6 \pm 0.1$ \\
$\left|\boldsymbol{r}_{\mathrm{DV} 1}-\boldsymbol{r}_{\mathrm{DV} 2}\right|>160 \mu \mathrm{m}$ & $0.2 \pm 0.01$ & $2.9 \pm 0.1$ & $5.1 \pm 0.1$ & $5.9 \pm 0.1$ \\
$\left|\boldsymbol{r}_{\mathrm{DV} 1}-\boldsymbol{r}_{\mathrm{DV} 2}\right|>200 \mu \mathrm{m}$ & $<0.1$ & $2.2 \pm 0.1$ & $4.5 \pm 0.1$ & $5.6 \pm 0.1$ \\
\hline
\end{tabular}

(b) The expected number of signal events for $m_{\tilde{g}}=2800 \mathrm{GeV}$ and $m_{\tilde{\chi}_{1}^{0}}=100 \mathrm{GeV}$ with different values of $c \tau_{\tilde{g}}$.

Table 7. The expected number of background (a) and signal (b) events for an integrated luminosity of $\mathcal{L}=3000 \mathrm{fb}^{-1}$. We set the masses of gluino and the LSP to be $2800 \mathrm{GeV}$ and $100 \mathrm{GeV}$, respectively.

\begin{tabular}{|l|ccc|c|}
\hline & $Z$ & $W$ & $t \bar{t}$ & total \\
\hline preselection-M $\left(\times 10^{4}\right)$ & $1.7 \pm 0.10$ & $1.7 \pm 0.22$ & $1.8 \pm 0.05$ & $5.2 \pm 0.25$ \\
$m_{\text {eff }}$ (incl. $)>2400 \mathrm{GeV}$ & $357 \pm 41$ & $148 \pm 13$ & $37 \pm 5$ & $542 \pm 44$ \\
\hline$\left|\boldsymbol{r}_{\mathrm{DV} 1}-\boldsymbol{r}_{\mathrm{DV} 2}\right|>160 \mu \mathrm{m}$ & $8.2 \pm 4$ & $3.6 \pm 1$ & $0.5 \pm 0.2$ & $12.3 \pm 4$ \\
$\left|\boldsymbol{r}_{\mathrm{DV} 1}-\boldsymbol{r}_{\mathrm{DV} 2}\right|>320 \mu \mathrm{m}$ & $0.2 \pm 0.1$ & $<0.1$ & $0.2 \pm 0.2$ & $0.5 \pm 0.2$ \\
$\left|\boldsymbol{r}_{\mathrm{DV} 1}-\boldsymbol{r}_{\mathrm{DV} 2}\right|>400 \mu \mathrm{m}$ & $<0.1$ & $<0.1$ & $<0.1$ & $<0.1$ \\
\hline
\end{tabular}

(a) The expected number of background events.

\begin{tabular}{|l|cccc|}
\hline & $c \tau_{\tilde{g}}=0$ & $c \tau_{\tilde{g}}=200 \mu \mathrm{m}$ & $c \tau_{\tilde{g}}=500 \mu \mathrm{m}$ & $c \tau_{\tilde{g}}=1 \mathrm{~mm}$ \\
\hline preselection-M & \multicolumn{5}{|c|}{$68.6 \pm 0.7$} \\
$m_{\mathrm{eff}}($ incl.) $>2400 \mathrm{GeV}$ & \multicolumn{4}{|c}{$31.2 \pm 0.5$} \\
\hline$\left|\boldsymbol{r}_{\mathrm{DV} 1}-\boldsymbol{r}_{\mathrm{DV} 2}\right|>160 \mu \mathrm{m}$ & $0.8 \pm 0.1$ & $12.7 \pm 0.3$ & $21.1 \pm 0.4$ & $24.1 \pm 0.4$ \\
$\left|\boldsymbol{r}_{\mathrm{DV} 1}-\boldsymbol{r}_{\mathrm{DV} 2}\right|>320 \mu \mathrm{m}$ & $<0.1$ & $4.6 \pm 0.2$ & $13.9 \pm 0.3$ & $18.9 \pm 0.4$ \\
$\left|\boldsymbol{r}_{\mathrm{DV} 1}-\boldsymbol{r}_{\mathrm{DV} 2}\right|>400 \mu \mathrm{m}$ & $<0.1$ & $2.5 \pm 0.1$ & $10.2 \pm 0.3$ & $16.2 \pm 0.4$ \\
\hline
\end{tabular}

(b) The expected number of signal events for $m_{\tilde{g}}=2400 \mathrm{GeV}, m_{\tilde{\chi}_{1}^{0}}=1440 \mathrm{GeV}$ with different values of $c \tau_{\tilde{g}}$.

Table 8. The same as in table 7 but preselection-M is imposed and the masses of gluino and the LSP are set to be $2400 \mathrm{GeV}$ and $1440 \mathrm{GeV}$, respectively. 


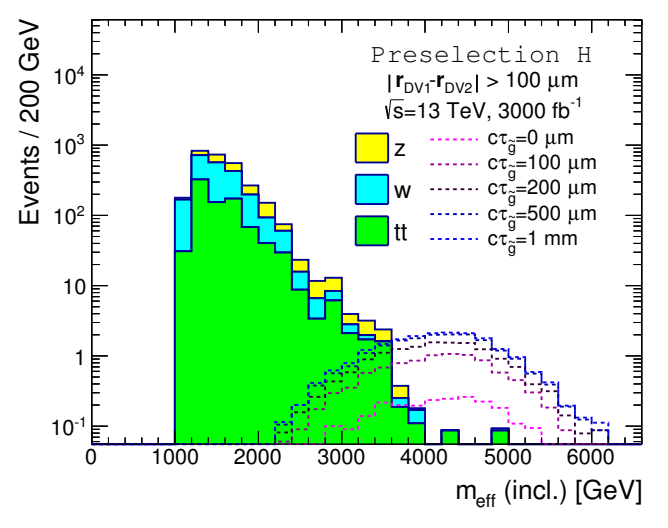

(a) $\left|\boldsymbol{r}_{\mathrm{DV} 1}-\boldsymbol{r}_{\mathrm{DV} 2}\right|>100 \mu \mathrm{m}$

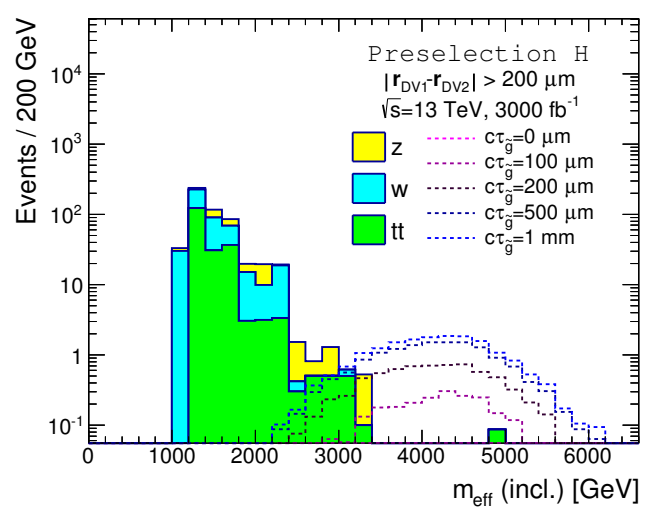

(b) $\left|\boldsymbol{r}_{\mathrm{DV} 1}-\boldsymbol{r}_{\mathrm{DV} 2}\right|>200 \mu \mathrm{m}$

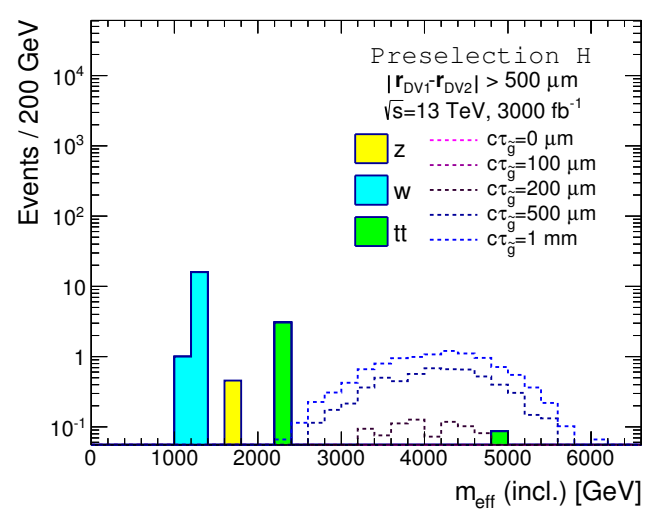

(c) $\left|\boldsymbol{r}_{\mathrm{DV} 1}-\boldsymbol{r}_{\mathrm{DV} 2}\right|>500 \mu \mathrm{m}$

Figure 8. Distributions of $m_{\mathrm{eff}}$ (incl.) for the SM background and signal events with different values of $c \tau_{\tilde{g}}$. The masses of gluino and the LSP are set to be $2600 \mathrm{GeV}$ and $100 \mathrm{GeV}$, respectively. We have imposed preselection- $\mathrm{H}$ and the vetoes given in table 6 .

of the new selection cut may be seen by comparing the reach for each $c \tau_{\tilde{g}}$ with that for $c \tau_{\tilde{g}}=0$, for which the new selection cut is ineffective as seen from figure 7a. It turns out that, even with the current data, the exclusion limit can be improved by about 100 and $120 \mathrm{GeV}$ for $c \tau_{\tilde{g}}=0.5$ and $1 \mathrm{~mm}$, respectively. We also find that the sensitivity gets worse for $c \tau_{\tilde{g}} \gtrsim \mathcal{O}(10) \mathrm{mm}$. This is because the signal efficiency is decreased due to the requirements on the production point and impact parameters of tracks as well as the detector material veto on the position of reconstructed vertices. To compare the result with the current sensitivities of other gluino searches, we also show the $95 \%$ CL exclusion limits given by the ATLAS prompt-decay gluino search with the $13 \mathrm{TeV} 36.1 \mathrm{fb}^{-1}$ data (black dotted line) [144], the ATLAS displaced-vertex search with the $13 \mathrm{TeV} 32.8 \mathrm{fb}^{-1}$ data $^{-}$ (blue dashed line) [67], and the ATLAS search of large ionization energy loss in the Pixel detector with the $13 \mathrm{TeV} 3.2 \mathrm{fb}^{-1}$ data (green dot-dashed line) [73]. Note that we extend the black dotted line for the ATLAS prompt-decay gluino search up to $c \tau_{\tilde{g}} \sim \mathcal{O}(1) \mathrm{mm}$ just for comparison; the reach of the prompt-decay gluino search is expected to become 


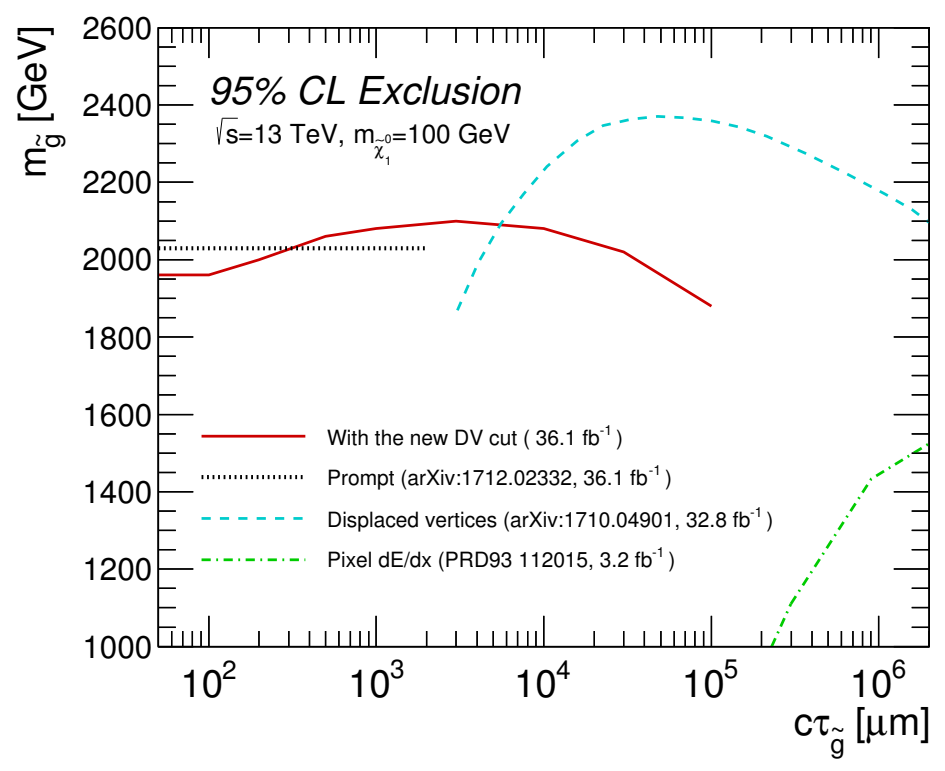

Figure 9. The $95 \% \mathrm{CL}$ expected exclusion limit on the gluino mass with $\mathcal{L}=36.1 \mathrm{fb}^{-1}$ at the $13 \mathrm{TeV}$ LHC run as a function of $c \tau_{\tilde{g}}$ (red solid line). For comparison, we also show the $95 \% \mathrm{CL}$ exclusion limits given by the ATLAS prompt-decay gluino search (black dotted line) [144], the ATLAS displaced-vertex search (blue dashed line) [67], and the ATLAS search of large ionization energy loss in the Pixel detector (green dot-dashed line) [73].

worse for $c \tau_{\tilde{g}} \gtrsim \mathcal{O}(1) \mathrm{mm}[159] .{ }^{11}$ We see that the existing metastable gluino searches are insensitive to a gluino with $c \tau_{\tilde{g}} \lesssim 1 \mathrm{~mm}$, where our event-selection criterion may offer a good sensitivity. In this sense, this new search strategy plays a complementary role in probing metastable gluinos.

In figure 10a, we show the expected 95\% CL exclusion limits (in dotted lines) and $5 \sigma$ discovery reaches (in solid lines) for gluino as functions of $c \tau_{\tilde{g}}$ for different values of integrated luminosity at the $13 \mathrm{TeV}$ LHC run, where the LSP mass is set to be $100 \mathrm{GeV}$. Notice that the expected reaches for an extremely small $c \tau_{\tilde{g}}$ should correspond to those for the prompt-decay gluino search with the same data set since the new selection cut plays no role in this case. As can be seen from this figure, the reach for the gluino can be extended with the help of the new selection cut for $c \tau_{\tilde{g}} \gtrsim 100 \mu \mathrm{m}$; e.g., for a gluino with $c \tau_{\tilde{g}} \sim \mathcal{O}(1-10) \mathrm{mm}$, the expected discovery reach for the gluino mass can be extended by as large as $\sim 240 \mathrm{GeV}(320 \mathrm{GeV})$ with an integrated luminosity of $\mathcal{L}=300 \mathrm{fb}^{-1}\left(3000 \mathrm{fb}^{-1}\right)$. These reaches for a gluino with $c \tau_{\tilde{g}} \sim \mathcal{O}(1-10) \mathrm{mm}$ are obtained with preselection $\mathrm{H},\left(m_{\text {effcut }}\right)_{\text {optimal }}=2400 \mathrm{GeV}(3400 \mathrm{GeV})$, and $\left(r_{\text {cut }}\right)_{\text {optimal }} \sim 300 \mu \mathrm{m}(160 \mu \mathrm{m})$ for $\mathcal{L}=$ $300 \mathrm{fb}^{-1}\left(3000 \mathrm{fb}^{-1}\right)$. Compared to a promptly decaying gluino, for which $\left(m_{\text {effcut }}\right)_{\text {optimal }}=$ $3400 \mathrm{GeV}(4200 \mathrm{GeV})$, the $m_{\text {eff }}$ (incl.) selection cut is found to be significantly loosened,

\footnotetext{
${ }^{11}$ In the gluino search performed by the CMS in ref. [160], the sensitivity is maximized for $c \tau_{\tilde{g}} \simeq \mathcal{O}(1) \mathrm{mm}$ and then gets worse for larger values of $c \tau_{\tilde{g}}$. The good sensitivity at $c \tau_{\tilde{g}} \simeq \mathcal{O}(1) \mathrm{mm}$ is due to the CSV tag [161] for the $b$ tagging. This result indeed indicates that an elaborated vertex reconstruction algorithm can improve the sensitivity of metastable gluino searches, as we discuss in the present paper.
} 


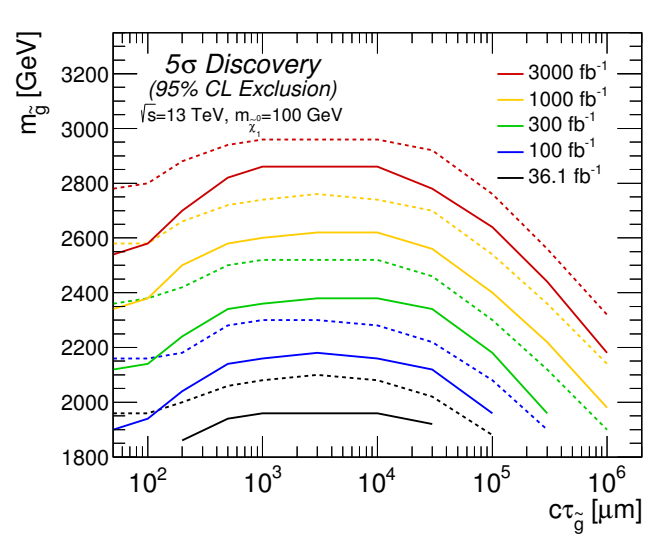

(a) $m_{\tilde{\chi}_{1}^{0}}=100 \mathrm{GeV}$

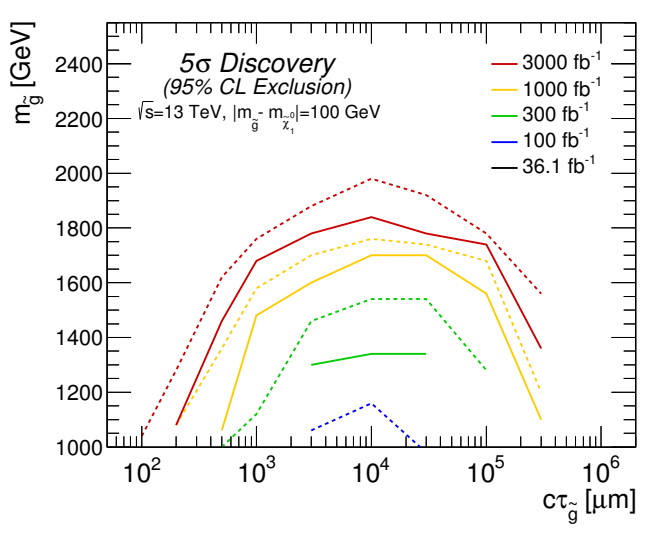

(b) $\left|m_{\tilde{g}}-m_{\tilde{\chi}_{1}^{0}}\right|=100 \mathrm{GeV}$

Figure 10. The expected $95 \%$ CL exclusion limits (dotted) and $5 \sigma$ discovery reaches (solid) as functions of $c \tau_{\tilde{g}}$ for different values of integrated luminosity at the $13 \mathrm{TeV} \mathrm{LHC}$ run.

which implies that the new selection cut plays an important role in background rejection. As we already mentioned, because charged tracks with $\left|d_{0}\right|>10 \mathrm{~mm}$ are not included in the analysis, and also because we reject all events in which a vertex is reconstructed with a position radius larger than $120 \mathrm{~mm}$, the expected exclusion limits decrease for $c \tau_{\tilde{g}} \gtrsim 100 \mathrm{~mm}$. Such a larger $c \tau_{\tilde{g}}$ region can however be covered by other long-lived gluino searches.

We also study the case where gluino and the LSP are degenerate in mass, which is motivated by the coannihilation scenario as we mentioned in section 3 . The result is shown in figure 10b. Here, the mass difference between gluino and the LSP is set to be $100 \mathrm{GeV}$. We find that the limits and the reaches are strongly enhanced especially for $c \tau_{\tilde{g}} \sim \mathcal{O}(1-100) \mathrm{mm}$. Contrary to the previous case, we are unable to set a reach or limit higher than $1000 \mathrm{GeV}$ for low luminosities. A caveat here is that we impose relatively tight requirements on jet $p_{\mathrm{T}}$ and thus this analysis is not optimized for the degenerate mass region. Indeed, according to the analysis done by the ATLAS collaboration [162], the event selection category called Meff-5j-1400 provides the best sensitivity for the degenerate mass region, where conditions on $p_{\mathrm{T}}$ for $2 \mathrm{nd}-4$ th jets are relaxed and another 5 th jet is required instead. We however do not try to further explore such an optimization in this paper - as the number of additional partons in our MC simulation is restricted to less than five - and defer it to another occasion.

Finally, we present the $5 \sigma$ discovery reaches and expected 95\% CL exclusion limits for various values of $c \tau_{\tilde{g}}$ in figures 11,12 , and 13 for an integrated luminosity of 36.1,300, and $3000 \mathrm{fb}^{-1}$, respectively. In figure 11b, we also show the expected $95 \% \mathrm{CL}$ exclusion limit given by the ATLAS experiment [144] in the black dotted line. We see that this ATLAS limit is in a fairly good agreement with our limit for $c \tau_{\tilde{g}}=0 \mu \mathrm{m}$ shown in the black solid line, besides the regions where gluino and the LSP are highly degenerate in mass. This discrepancy is expected since our analysis is not optimized for the degenerate region as we discussed above. From these plots, we see that an implementation of an extra cut utilizing 


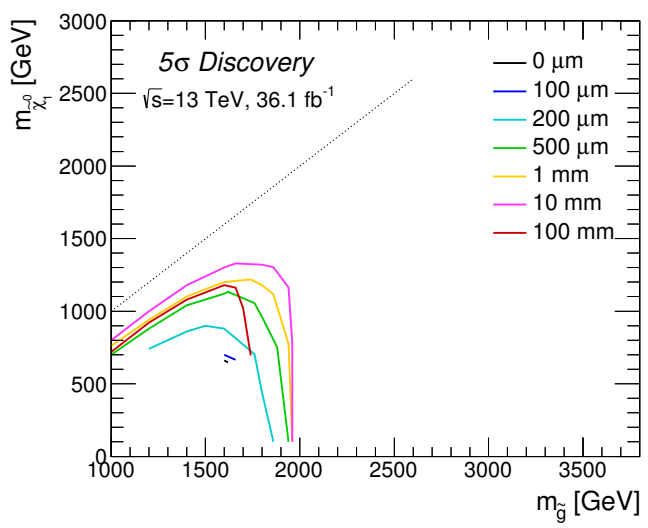

(a) $5 \sigma$ discovery

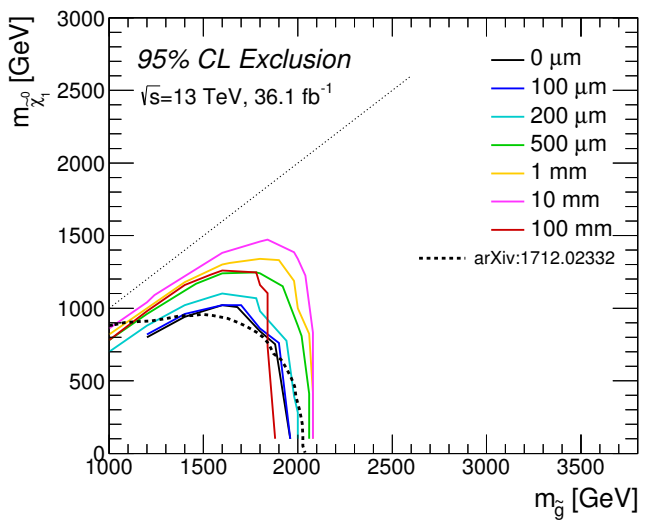

(b) Expected 95\% CL exclusion

Figure 11. $5 \sigma$ discovery reaches and expected $95 \%$ CL exclusion limits for gluinos with different $c \tau_{\tilde{q}}$, for an integrated luminosity of $36.1 \mathrm{fb}^{-1}$. The expected $95 \%$ CL exclusion limit from the ATLAS promptly-decaying gluino search [144] is also shown in the black dotted line.

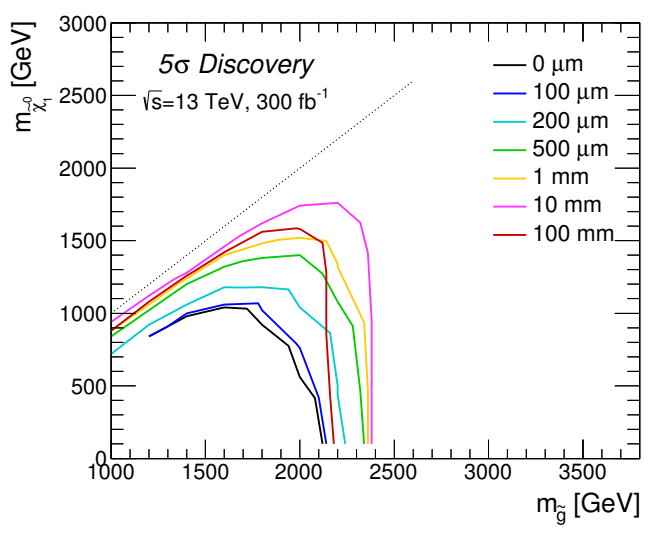

(a) $5 \sigma$ discovery

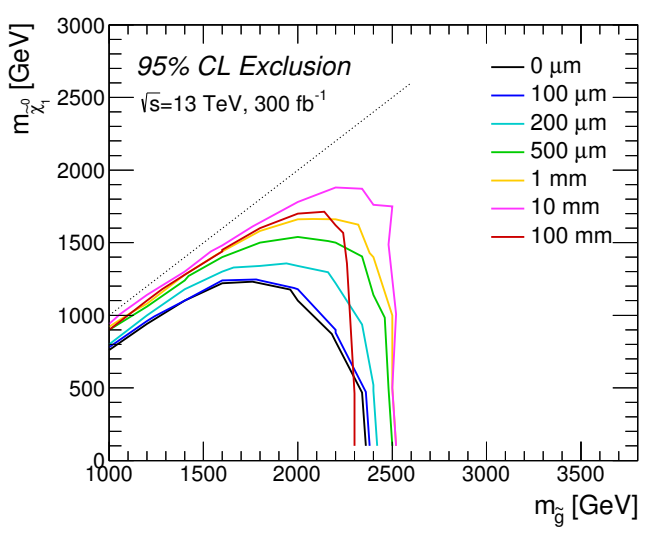

(b) Expected 95\% CL exclusion

Figure 12. Same as in figure 11, but for an integrated luminosity of $300 \mathrm{fb}^{-1}$.

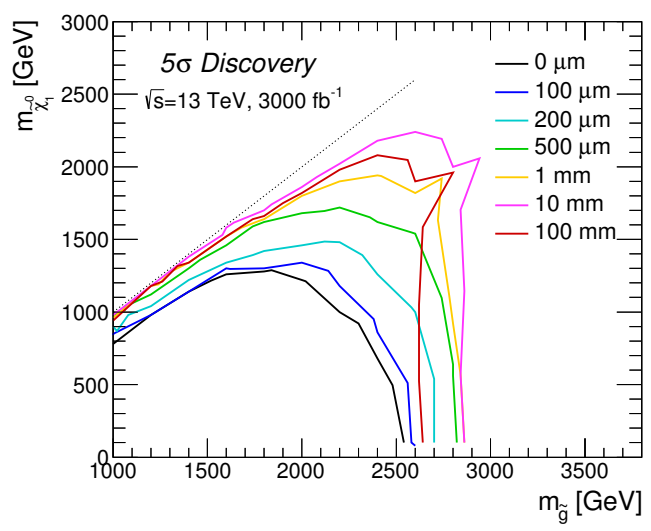

(a) $5 \sigma$ discovery

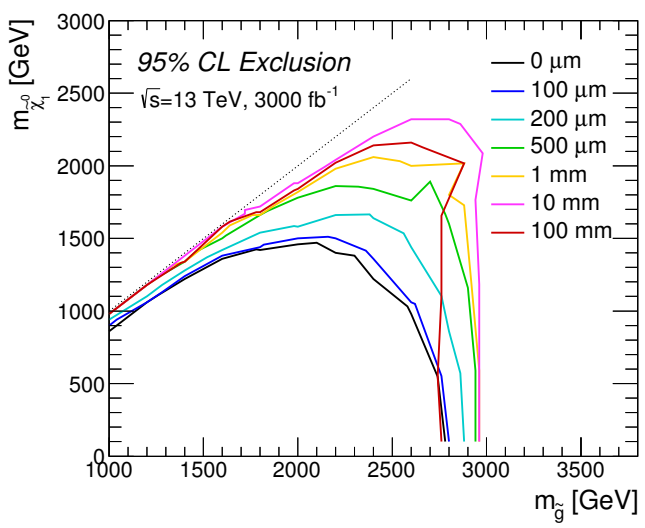

(b) Expected $95 \%$ CL exclusion

Figure 13. Same as in figure 11, but for an integrated luminosity of $3000 \mathrm{fb}^{-1}$. 
the vertex reconstruction leads to a significant improvement in both the discovery reach and the exclusion limit for $c \tau_{\tilde{g}} \gtrsim 200 \mu \mathrm{m}$. The extent of the improvement is maximized for $c \tau_{\tilde{g}} \sim \mathcal{O}(1-10) \mathrm{mm}$ and tends to get larger for a heavier LSP. This feature is also seen in figure 10, where we find that the improvement in the degenerate case (figure 10b) is much significant compared with the light LSP case (figure 10a). This follows from the fact that jets and the missing energy in gluino decays for the degenerate case tend to be soft, and thus traditional kinematical selection cuts become less powerful in this case. In such a situation, a sizable amount of the SM background remain after the kinematical selection cuts, but an additional cut based on vertex reconstruction can remove this efficiently, which results in a drastic improvement in the sensitivity.

\subsection{Lifetime measurements}

Once a new metastable particle is discovered at the LHC, the measurement of its lifetime is of crucial importance to understand the nature of new physics behind this metastable particle. For example, by measuring the lifetime of metastable gluino, we can infer the mass scale of squarks through eq. (3.1). In this subsection, we discuss the prospects of the lifetime measurement by means of the displaced-vertex reconstruction.

To see this, we study the expected significance of rejection of a hypothesis that the gluino decay length is $c \tau_{\tilde{g}}^{\text {(hypo) }}$ for gluino samples with a decay length of $c \tau_{\tilde{g}}$. Event samples are binned according to the distance between the two reconstructed vertices $\left|\boldsymbol{r}_{\mathrm{DV} 1}-\boldsymbol{r}_{\mathrm{DV} 2}\right|$ of the events. Then the expected significance $\left\langle Z_{c \tau_{\tilde{g}}^{(\text {hypo })}}\right\rangle_{c \tau_{\tilde{g}}}$ is defined by

$$
\left\langle Z_{c \tau_{\tilde{g}}^{(\text {hypo })}}\right\rangle_{c \tau_{\tilde{g}}} \equiv \sqrt{\Delta \chi^{2}\left(c \tau_{\tilde{g}}^{\text {(hypo })}, c \tau_{\tilde{g}}\right)}
$$

where

$$
\Delta \chi^{2}\left(c \tau_{\tilde{g}}^{\text {(hypo })}, c \tau_{\tilde{g}}\right)=\sum_{\operatorname{bin} i} \frac{\left\{S_{i}\left(c \tau_{\tilde{g}}^{\text {(hypo })}\right)-S_{i}\left(c \tau_{\tilde{g}}\right)\right\}^{2}}{S_{i}\left(c \tau_{\tilde{g}}^{(\text {hypo })}\right)+B_{i}} .
$$

Here, $S_{i}(c \tau)$ is the expected number of signal events in the bin $i$ on the assumption that gluinos have a decay length of $c \tau$, while $B_{i}$ is the number of SM background events.

In figures $14 \mathrm{a}$ and $14 \mathrm{~b}$, we show the expected upper and lower bounds on the decay length as a function of $c \tau_{\tilde{g}}$ for a gluino with a mass of $2.2 \mathrm{TeV}$. Here we impose preselection-H and require $m_{\text {eff }}$ (incl.) $>3500 \mathrm{GeV}$. From these figures, we find that a metastable gluino with $c \tau_{\tilde{g}} \gtrsim 30(60) \mu \mathrm{m}$ can be distinguished from a promptly decaying one with the significance of $2 \sigma(5 \sigma)$ with an integrated luminosity of $3000 \mathrm{fb}^{-1}$. Moreover, figure $14 \mathrm{~b}$ shows that the decay length of a gluino with $c \tau_{\tilde{g}} \sim \mathcal{O}(100) \mu \mathrm{m}$ can be measured with an $\mathcal{O}(1)$ accuracy at the high-luminosity LHC. With such a measurement, we may probe the squark mass scale $m_{\tilde{q}}$ via eq. (3.1) even though squarks are inaccessible at the LHC. We also note that even if only an upper limit on the gluino decay length is obtained, this gives valuable implications for SUSY models, since such a limit results in an upper bound on the squark mass scale; e.g., for $m_{\tilde{g}}=2.2 \mathrm{TeV}$, an upper limit on the decay length 


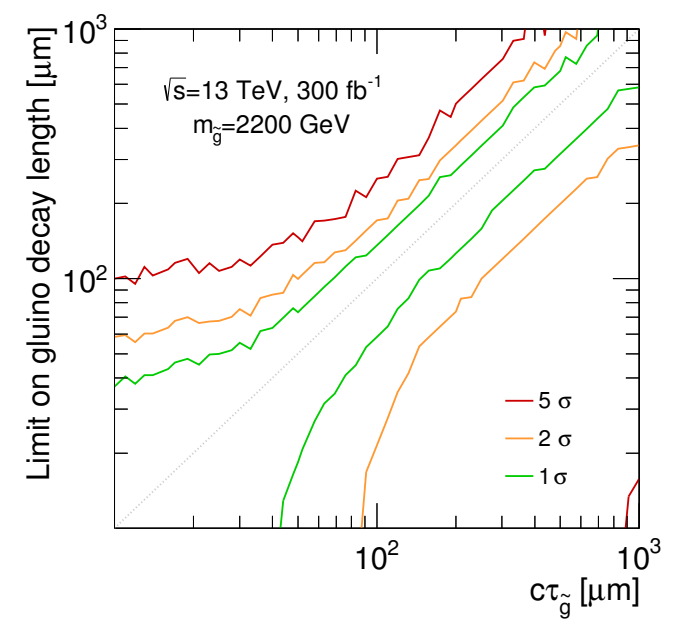

(a) $\mathcal{L}=300 \mathrm{fb}^{-1}$

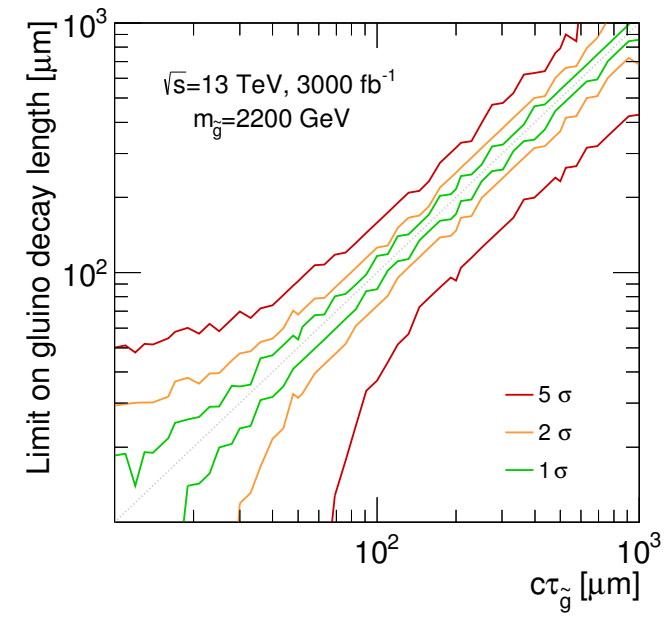

(b) $\mathcal{L}=3000 \mathrm{fb}^{-1}$

Figure 14. The expected upper and lower bounds on the decay length of gluino as a function of the underlying value of $c \tau_{\tilde{g}}$. Here, we set $m_{\tilde{g}}=2.2 \mathrm{TeV}$, and impose preselection- $\mathrm{H}$ and a selection cut $m_{\text {eff }}$ (incl.) $>3500 \mathrm{GeV}$.

$\left.c \tau_{\tilde{g}}\right|_{\text {limit }}$ leads to

$$
m_{\tilde{q}} \lesssim 10^{3} \times\left(\frac{\left.c \tau_{\tilde{g}}\right|_{\text {limit }}}{100 \mu \mathrm{m}}\right)^{\frac{1}{4}} \mathrm{TeV} .
$$

After all, the reconstruction of sub-millimeter decay vertices is quite important even after the discovery of a new particle such as gluino, as its lifetime contains precious information on the underlying physics.

\section{$5 \quad 100 \mathrm{TeV}$ collider}

The vertex-reconstruction method developed above can also be applied to other collider experiments. Recently, there have been a growing interest in future collider experiments with a center-of-mass energy higher than those at the LHC, such as a $100 \mathrm{TeV}$ collider [79, 80]. Motivated by this, in this section we apply our new selection cut to the searches for metastable gluinos at a future $100 \mathrm{TeV} p p$ collider and study how much this new selection cut can improve the sensitivity of the prompt-decay searches in this case. As we have already seen in section 3, the production cross section of gluinos at a $100 \mathrm{TeV}$ collider is much larger than those at the LHC, which drastically extends the reach of gluino searches $[79,80,145,163]$. In addition, particles tend to be produced in a highly boosted state at a $100 \mathrm{TeV}$ collider, which then prolongs the lifetime of metastable particles - this allows us to probe particles with shorter decay length, as we actually see in the following analysis.

\subsection{MC simulation}

For MC simulation of a $100 \mathrm{TeV}$ collider, we basically follow the same procedure as described in section 4.1. We list the sample points for signal events in table $9,{ }^{12}$ with the same

\footnotetext{
${ }^{12}$ For $m_{\tilde{g}}=18 \mathrm{TeV}$, we only generate samples with $m_{\tilde{\chi}_{1}^{0}}=100 \mathrm{GeV}$.
} 


\begin{tabular}{|c|c|}
\hline$m_{\tilde{g}}$ & $3,4, \ldots 6,8, \ldots 16,18 \mathrm{TeV}$ \\
\hline \multirow{3}{*}{$m_{\tilde{\chi}_{1}^{0}}$} & $100 \mathrm{GeV}$, \\
& $m_{\tilde{g}}-(150 \mathrm{GeV}, 100 \mathrm{GeV}, 50 \mathrm{GeV}, 25 \mathrm{GeV})$ \\
\hline$c \tau_{\tilde{g}}$ & $0,50,100,200,500,1000,3000 \mu \mathrm{m}$, \\
& $1 \times 10^{4}, 3 \times 10^{4}, 1 \times 10^{5}, 3 \times 10^{5}, 1 \times 10^{6} \mu \mathrm{m}$ \\
\hline
\end{tabular}

Table 9. Sample points for signal events. We generate 50000 events for each sample point.

\begin{tabular}{|cc|ccccc|}
\hline & bin $\alpha$ & 0 & 1 & 2 & 3 & 4 \\
\hline$H_{T, 0}^{\max }(\alpha)$ & $(\mathrm{GeV})$ & 900 & 2100 & 4000 & 6400 & $\infty$ \\
$\sigma_{\text {matched }}(\alpha)$ & $(\mathrm{pb})$ & $3.69 \times 10^{2}$ & 25.8 & 2.30 & 0.23 & 0.04 \\
$N_{\text {matched }}(\alpha)$ & $\left(\times 10^{3}\right)$ & 728 & 520 & 462 & 473 & 590 \\
$\mathcal{L}_{\text {gen }}(\alpha)$ & $\left(\mathrm{ab}^{-1}\right)$ & $1.97 \times 10^{-3}$ & 0.0201 & 0.201 & 2.02 & 14.5 \\
\hline
\end{tabular}

(a) $Z \rightarrow \nu \bar{\nu}+0,1,2,3 j$.

\begin{tabular}{|cc|ccccc|}
\hline & bin $\alpha$ & 0 & 1 & 2 & 3 & 4 \\
\hline$H_{T, 0}^{\max }(\alpha)$ & $(\mathrm{GeV})$ & 900 & 2100 & 4000 & 6400 & $\infty$ \\
$\sigma_{\text {matched }}(\alpha)$ & $(\mathrm{pb})$ & $1.20 \times 10^{3}$ & 22.4 & 1.70 & 0.16 & 0.03 \\
$N_{\text {matched }}(\alpha)$ & $\left(\times 10^{3}\right)$ & 121 & 90.9 & 82.9 & 79.3 & 75.2 \\
$\mathcal{L}_{\text {gen }}(\alpha)$ & $\left(\mathrm{ab}^{-1}\right)$ & $1.01 \times 10^{-4}$ & $4.06 \times 10^{-3}$ & 0.0487 & 0.496 & 2.95 \\
\hline
\end{tabular}

(b) $Z \rightarrow \ell \bar{\ell}+0,1,2,3 j$.

Table 10. The upper edge value of $H_{T, 0}$, the leading-order matched cross section, the number of samples after matching, and equivalent luminosity in each $H_{T, 0}$ bin for the $Z$-boson production process.

\begin{tabular}{|cc|cccccc|}
\hline & bin $\alpha$ & 0 & 1 & 2 & 3 & 4 & 5 \\
\hline$H_{T, 0}^{\max }(\alpha)$ & $(\mathrm{GeV})$ & 600 & 1500 & 3000 & 5000 & 7500 & $\infty$ \\
$\sigma_{\text {matched }}(\alpha)$ & $(\mathrm{pb})$ & $9.62 \times 10^{3}$ & $5.19 \times 10^{2}$ & 41.7 & 3.93 & 0.49 & 0.09 \\
$N_{\text {matched }}(\alpha)$ & $\left(\times 10^{3}\right)$ & 1910 & 1020 & 813 & 759 & 993 & 1950 \\
$\mathcal{L}_{\text {gen }}(\alpha)$ & $\left(\mathrm{ab}^{-1}\right)$ & $1.99 \times 10^{-4}$ & $1.97 \times 10^{-3}$ & 0.0195 & 0.193 & 2.05 & 20.6 \\
\hline
\end{tabular}

Table 11. Same as in table 10 but for the $W$-boson production process.

categorization as in table 1 . We again generate 50000 events for each sample point. For the SM background processes, we again focus on the $Z, W$, and $t \bar{t}$ production processes, which turn out to be dominant [145]. We carry out simulations with up to three and one additional partons for the $Z / W$ and $t \bar{t}$ production processes, respectively. In generating background samples, we divide the generator-level phase space in terms of $H_{T, 0}$; we show the binning of this division in tables 10-12 for each process with the leading-order matched cross section, the number of samples after matching, and the equivalent luminosity.

Using these samples, we generate distributions of $m_{\text {eff }}$ (incl.) for each process with Preselection-H imposed. For reconstructed objects such as jets, charged leptons, and 


\begin{tabular}{|cc|ccccccc|}
\hline & bin $\alpha$ & 0 & 1 & 2 & 3 & 4 & 5 & 6 \\
\hline$H_{T, 0}^{\max }(\alpha)$ & $(\mathrm{GeV})$ & 800 & 1600 & 2800 & 4600 & 7200 & 10000 & $\infty$ \\
$\sigma_{\text {matched }}(\alpha)$ & $(\mathrm{pb})$ & $4.40 \times 10^{3}$ & $3.54 \times 10^{2}$ & 33.7 & 3.40 & 0.34 & 0.03 & $5.18 \times 10^{-3}$ \\
$N_{\text {matched }}(\alpha)$ & $\left(\times 10^{3}\right)$ & 53.6 & 59.8 & 63.5 & 65.3 & 66.2 & 67.2 & 68 \\
$\mathcal{L}_{\text {gen }}(\alpha)$ & $\left(\mathrm{ab}^{-1}\right)$ & $1.22 \times 10^{-5}$ & $1.69 \times 10^{-4}$ & $1.89 \times 10^{-3}$ & 0.0192 & 0.197 & 2.23 & 13.1 \\
\hline
\end{tabular}

(a) $t \bar{t} \rightarrow$ (semi-leptonic) $+0,1 j$.

\begin{tabular}{|cc|ccccc|}
\hline & $\operatorname{bin} \alpha$ & 0 & 1 & 2 & 3 & 4 \\
\hline$H_{T, 0}^{\max }(\alpha)$ & $(\mathrm{GeV})$ & 1100 & 2300 & 3900 & 6000 & $\infty$ \\
$\sigma_{\text {matched }}(\alpha)$ & $(\mathrm{pb})$ & 54.4 & 6.57 & 0.56 & 0.06 & $6.90 \times 10^{-3}$ \\
$N_{\text {matched }}(\alpha)$ & $\left(\times 10^{3}\right)$ & 60.4 & 72.6 & 74.2 & 74.8 & 75.7 \\
$\mathcal{L}_{\text {gen }}(\alpha)$ & $\left(\mathrm{ab}^{-1}\right)$ & $1.11 \times 10^{-3}$ & $1.11 \times 10^{-2}$ & 0.133 & 1.36 & 11.0 \\
\hline
\end{tabular}

(b) $t \bar{t} \rightarrow$ (leptonic) $+0,1 j$.

Table 12. Same as in table 10 but for the $t \bar{t}$ production process.

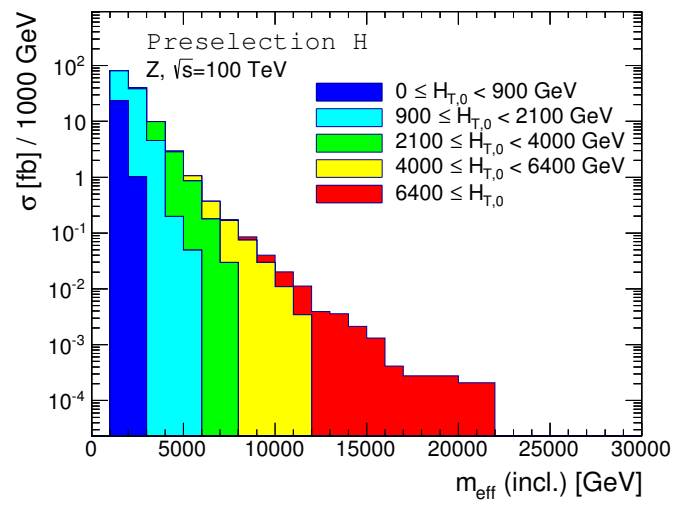

(a) $Z$ boson production

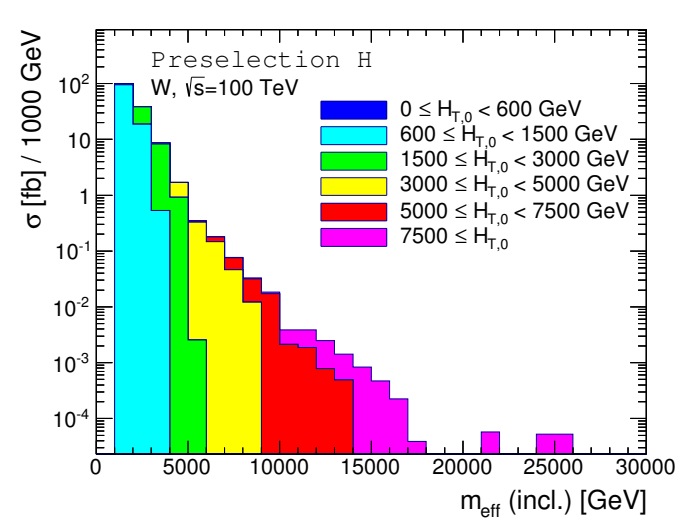

(b) $W$ boson production

Figure 15. Distributions of $m_{\text {eff }}$ (incl.) with preselection-H required. Contributions from different $H_{T, 0}$ bins are filled with different colors.

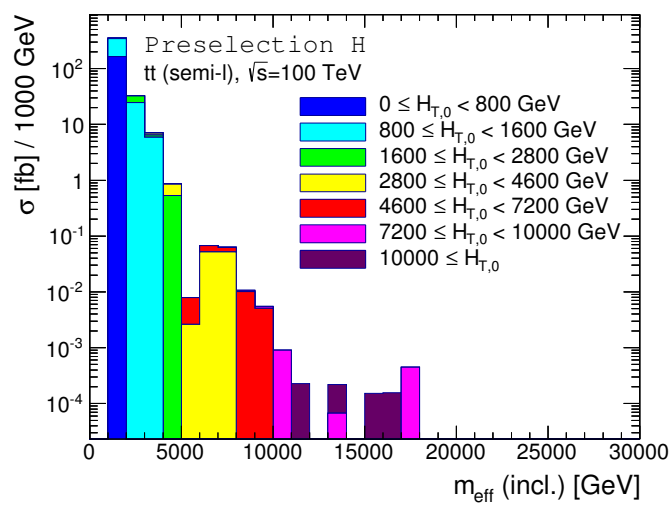

(a) Semi-leptonic $t \bar{t}$ decay

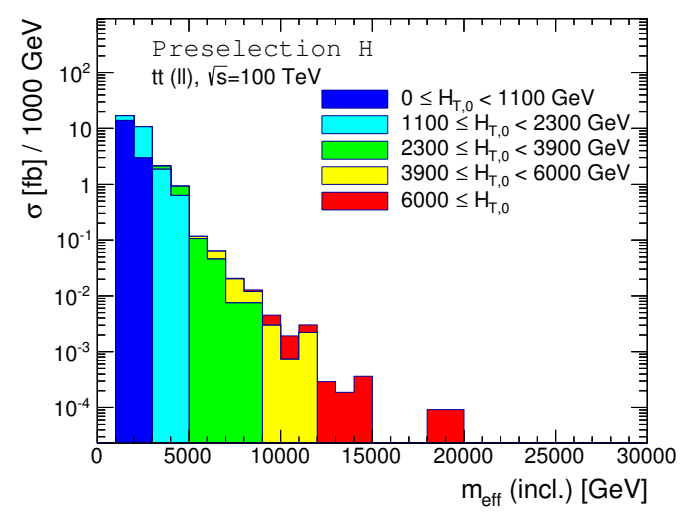

(b) Leptonic $t \bar{t}$ decay

Figure 16. Same as in figure 15, but for the $t \bar{t}$ production process. 


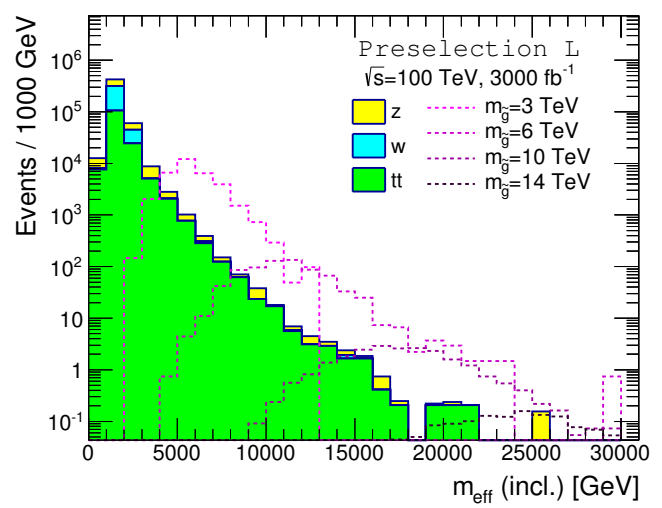

(a) Preselection-L

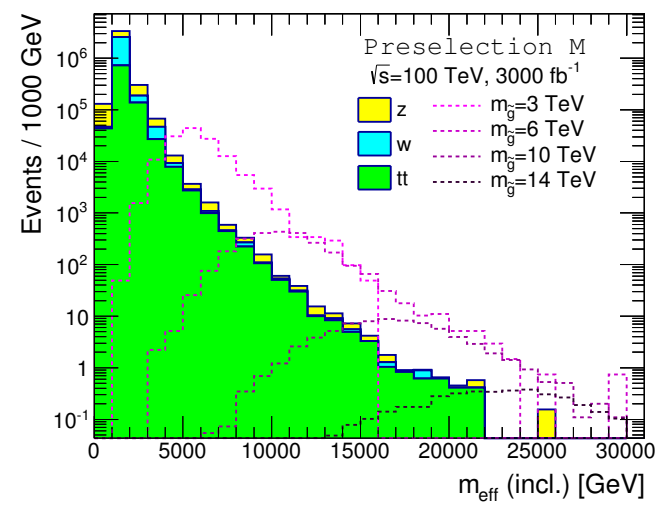

(b) Preselection-M

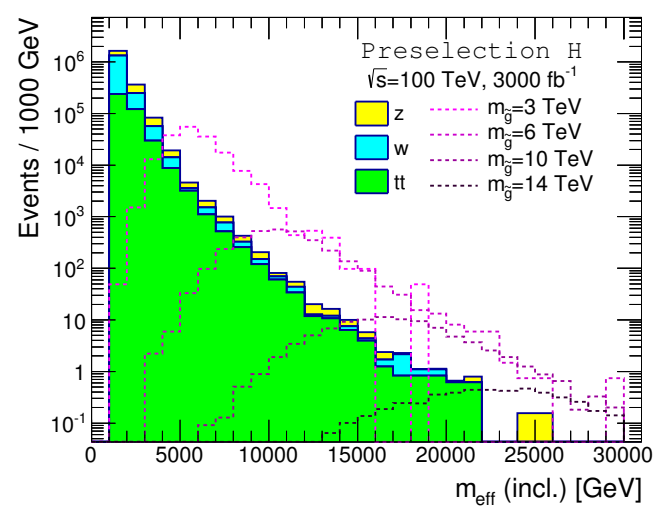

(c) Preselection-H

Figure 17. Distributions of $m_{\mathrm{eff}}$ (incl.) for the SM background and gluino signal events. We set the LSP mass to be $100 \mathrm{GeV}$.

charged tracks, we use the same criteria as in section 4.1. The resultant distributions are shown in figures 15 and 16. Again, we see a correlation between $H_{T, 0}$ and $m_{\text {eff }}$ (incl.) and a sizable number of events in the tails, which justifies our way of dividing the phase space in terms of $H_{T, 0}$.

To validate our MC simulation, we have compared the expected number of events obtained by our MC simulation with that given in ref. [145], with the same selection cuts adopted there. We have found a fairly good agreement $(\sim 20 \%$ level $)$ with each other over the signal regions.

\subsection{Event selection}

For the preselection, we consider the same criteria as in table 5. These criteria may be further optimized for a $100 \mathrm{TeV}$ collider, but we do not discuss this possibility in this paper. ${ }^{13}$ We again impose the lepton and detector-material vetoes. In figure 17 , we show

\footnotetext{
${ }^{13}$ As we noted in section 4.2 , even though the requirement for the $E_{\mathrm{T}}^{(\text {miss })}$ trigger is tightened at a future $100 \mathrm{TeV}$ collider, we expect that the event-selection efficiency is less affected since we also impose a large $m_{\text {eff }}$ (incl.) cut.
} 


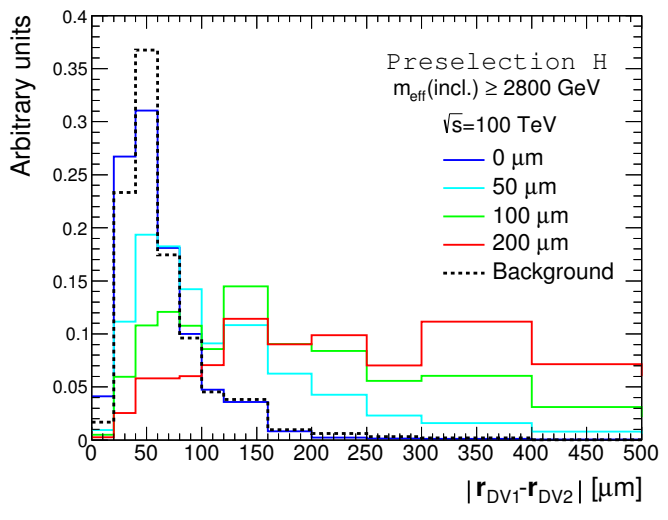

(a) $m_{\tilde{g}}=3 \mathrm{TeV}$

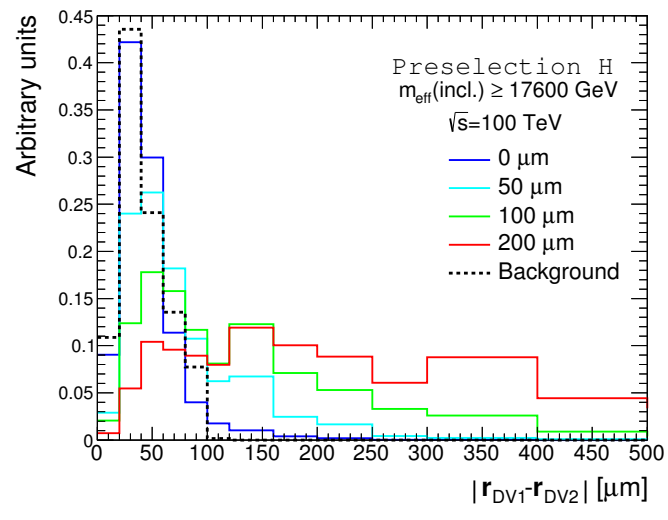

(b) $m_{\tilde{g}}=14 \mathrm{TeV}$

Figure 18. The distributions of $\left|\boldsymbol{r}_{\mathrm{DV} 1}-\boldsymbol{r}_{\mathrm{DV} 2}\right|$ for a gluino with different values of $c \tau_{\tilde{g}}$, shown in the solid lines. The distributions for the SM background events are also shown in the dotted lines. We have imposed preselection-H, and $m_{\mathrm{eff}}$ (incl.) $>2800 \mathrm{GeV}(17600 \mathrm{GeV})$ in figure 18a (figure 18b).

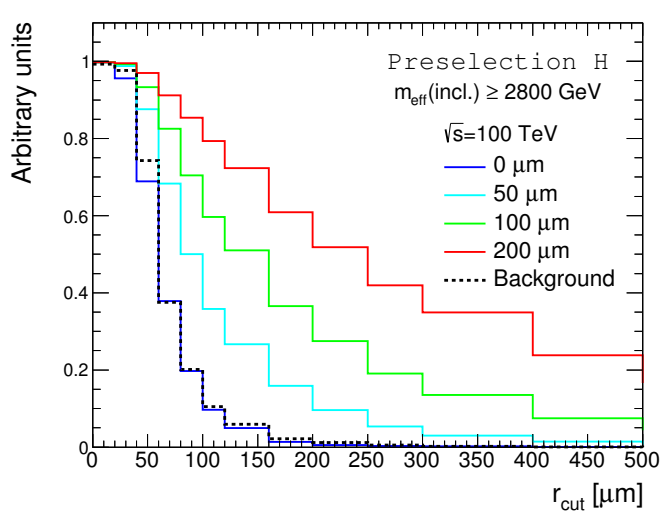

(a) $m_{\tilde{g}}=3 \mathrm{TeV}$

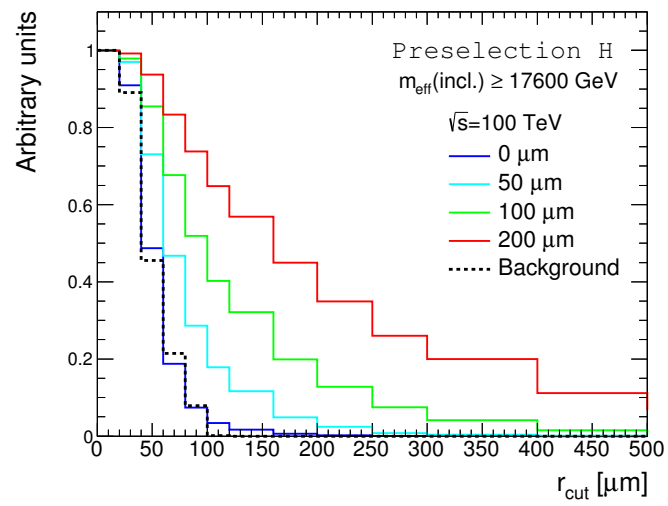

(b) $m_{\tilde{g}}=14 \mathrm{TeV}$

Figure 19. Fractions of events which pass the selection cut $\left|\boldsymbol{r}_{\mathrm{DV} 1}-\boldsymbol{r}_{\mathrm{DV} 2}\right|>r_{\text {cut }}$ for a gluino with different values of $c \tau_{\tilde{g}}$, shown in the solid lines. The distributions for the SM background events are also shown in the dotted lines. We have imposed preselection-H, and $m_{\text {eff }}$ (incl.) $>2800 \mathrm{GeV}$ $(17600 \mathrm{GeV}$ ) in figure 19a (figure 19b).

the distributions of $m_{\mathrm{eff}}$ (incl.) for the SM background and gluino signal events with each preselection imposed. We set the LSP mass to be $100 \mathrm{GeV}$ in these plots.

As we discuss in section 2 , the resolution of the vertex reconstruction highly depends on the track reconstruction performance of a detector. Due to a lack of concrete information on detectors at future $100 \mathrm{TeV}$ colliders, in the following analysis, we just assume the same track-resolution parameters as those given in section 4.2, with expecting that the detectors will be designed/developed such that their performance is maintained (or improved) in a similar level to the ATLAS and CMS detectors, and reconstruct vertices in the same manner as before. We further recall that our vertex reconstruction method is less sensitive to the number of pile-up events, as discussed in section 4.2 - we therefore neglect the effect 


\begin{tabular}{|l|ccc|c|}
\hline & $Z$ & $W$ & $t \bar{t}$ & total \\
\hline preselection-H $\left(\times 10^{6}\right)$ & $0.42 \pm 0.01$ & $0.45 \pm 0.02$ & $1.26 \pm 0.4$ & $2.13 \pm 0.4$ \\
$m_{\text {eff }}$ (incl. $)>17.8 \mathrm{TeV}$ & $3.1 \pm 0.8$ & $0.9 \pm 0.3$ & $0.5 \pm 0.4$ & $4.6 \pm 0.9$ \\
\hline$\left|\boldsymbol{r}_{\mathrm{DV} 1}-\boldsymbol{r}_{\mathrm{DV} 2}\right|>100 \mu \mathrm{m}$ & $<0.1$ & $<0.1$ & $<0.1$ & $<0.1$ \\
$\left|\boldsymbol{r}_{\mathrm{DV} 1}-\boldsymbol{r}_{\mathrm{DV} 2}\right|>200 \mu \mathrm{m}$ & $<0.1$ & $<0.1$ & $<0.1$ & $<0.1$ \\
\hline
\end{tabular}

(a) The expected number of background events.

\begin{tabular}{|l|cccc|}
\hline & $c \tau_{\tilde{g}}=0$ & $c \tau_{\tilde{g}}=200 \mu \mathrm{m}$ & $c \tau_{\tilde{g}}=500 \mu \mathrm{m}$ & $c \tau_{\tilde{g}}=1 \mathrm{~mm}$ \\
\hline preselection-H & \multicolumn{4}{|c|}{$20.5 \pm 0.3$} \\
$m_{\text {eff }}$ (incl.) $>17.8 \mathrm{TeV}$ & \multicolumn{4}{|c|}{$14.5 \pm 0.2$} \\
\hline$\left|\boldsymbol{r}_{\mathrm{DV} 1}-\boldsymbol{r}_{\mathrm{DV} 2}\right|>100 \mu \mathrm{m}$ & $0.5 \pm 0.04$ & $9.8 \pm 0.2$ & $12.5 \pm 0.2$ & $12.9 \pm 0.2$ \\
$\left|\boldsymbol{r}_{\mathrm{DV} 1}-\boldsymbol{r}_{\mathrm{DV} 2}\right|>200 \mu \mathrm{m}$ & $<0.1$ & $5.5 \pm 0.1$ & $9.6 \pm 0.2$ & $11.3 \pm 0.2$ \\
\hline
\end{tabular}

(b) The expected number of signal events for $m_{\tilde{g}}=12 \mathrm{TeV}, m_{\tilde{\chi}_{1}^{0}}=100 \mathrm{GeV}$ with different values of $c \tau_{\tilde{g}}$.

Table 13. The expected number of background (a) and signal (b) events for an integrated luminosity of $\mathcal{L}=3000 \mathrm{fb}^{-1}$. We set the masses of gluino and the LSP to be $12 \mathrm{TeV}$ and $100 \mathrm{GeV}$, respectively.

of pile-up events in the following analysis. We show the distributions of $\left|\boldsymbol{r}_{\mathrm{DV} 1}-\boldsymbol{r}_{\mathrm{DV} 2}\right|$ in the solid lines for a gluino with different values of $c \tau_{\tilde{g}}$ and a mass of $3 \mathrm{TeV}$ and $14 \mathrm{TeV}$ in figures 18a and 18b, respectively. Moreover, we show in figures 19a and 19b fractions of events which pass a selection cut of $\left|\boldsymbol{r}_{\mathrm{DV} 1}-\boldsymbol{r}_{\mathrm{DV} 2}\right|>r_{\text {cut }}$ as functions of $r_{\text {cut }}$. The distributions for the SM background events are also shown in the dotted lines. Here, we have imposed preselection-H, and $m_{\text {eff }}$ (incl.) $>2800 \mathrm{GeV}(17600 \mathrm{GeV})$ in the left (right) panel. By comparing these figures with figure 6, we clearly see the effect of Lorentz boost of metastable particles on the displaced-vertex reconstruction. In figure 6 , the distribution for the $c \tau_{\tilde{g}}=50 \mu \mathrm{m}$ case is found to be fairly close to those for $c \tau_{\tilde{g}}=0 \mu \mathrm{m}$ and the SM background. On the other hand, as seen in figures 18a and 19a, we can easily distinguish $c \tau_{\tilde{g}}=50 \mu \mathrm{m}$ from $c \tau_{\tilde{g}}=0 \mu \mathrm{m}$ and the SM background at a $100 \mathrm{TeV}$ collider. For a heavier gluino, however, the separation becomes less clear due to reduction in the boost factor.

\subsection{Prospects}

Now we discuss the prospects of our new selection cut for a $100 \mathrm{TeV}$ collider. We again use the event selection criteria summarized in table 6 , except that we now raise the upper limit on $m_{\text {effcut }}$ to $3 \times 10^{4} \mathrm{GeV}$. In figure 20 , we show optimal cut values $\left(\left(m_{\text {effcut }}\right)_{\text {optimal }}\right.$, $\left.\left(r_{\text {cut }}\right)_{\text {optimal }}\right)$, the preselection ( $\mathrm{L}, \mathrm{M}$, or $\left.\mathrm{H}\right)$, and the discriminator $\Delta r_{\mathrm{DV}}(\mathrm{R}, \mathrm{T}$, or $\mathrm{Z})$ for each sample point with various $c \tau_{\tilde{g}}$, for a $100 \mathrm{TeV}$ collider with an integrated luminosity of $3000 \mathrm{fb}^{-1}$. The expected $5 \sigma$ discovery reaches for gluinos are also shown in the dashed lines. We again find that the kinematic selection cut on $m_{\text {eff }}$ (incl.) may be highly relaxed with the help of the displaced-vertex cut with $\left(r_{\text {cut }}\right)_{\text {optimal }} \simeq c \tau_{\tilde{g}}$. In tables 13 and 14 , we present the expected number of background and signal events for an integrated luminosity of $\mathcal{L}=3000 \mathrm{fb}^{-1}$. In table $13(14)$, we consider the case of a light (heavy) LSP with $m_{\tilde{g}}=$ 


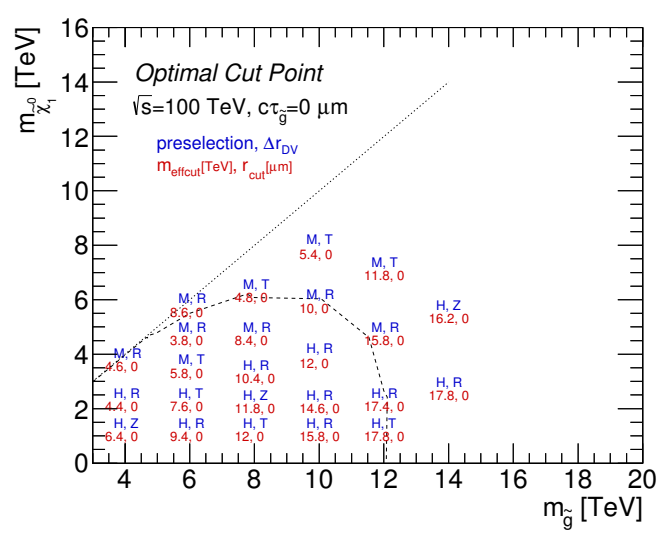

(a) $c \tau_{\tilde{g}}=0 \mu \mathrm{m}$

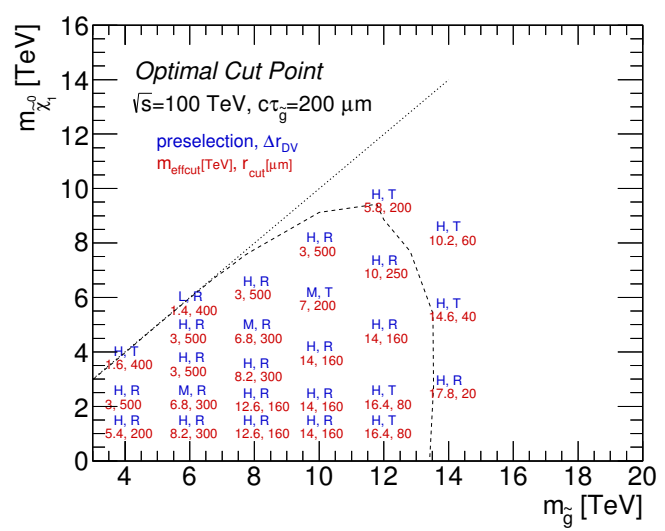

(c) $c \tau_{\tilde{g}}=200 \mu \mathrm{m}$

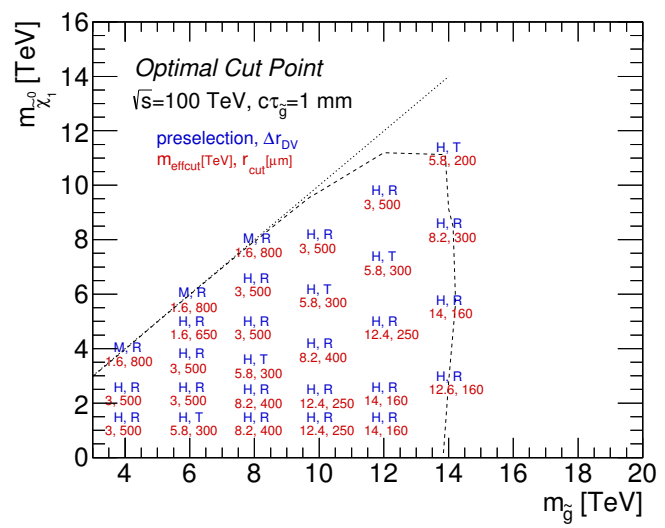

(e) $c \tau_{\tilde{g}}=1 \mathrm{~mm}$

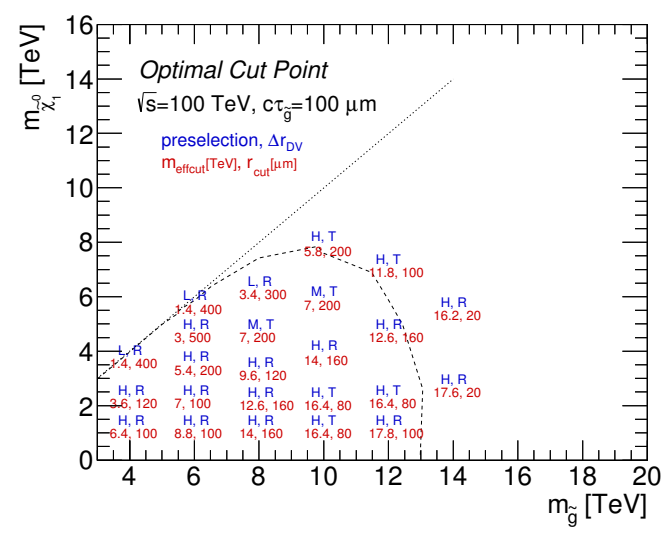

(b) $c \tau_{\tilde{g}}=100 \mu \mathrm{m}$

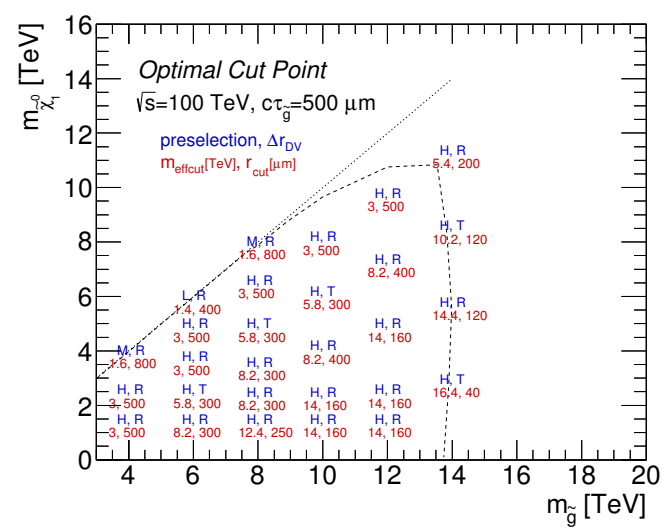

(d) $c \tau_{\tilde{g}}=500 \mu \mathrm{m}$

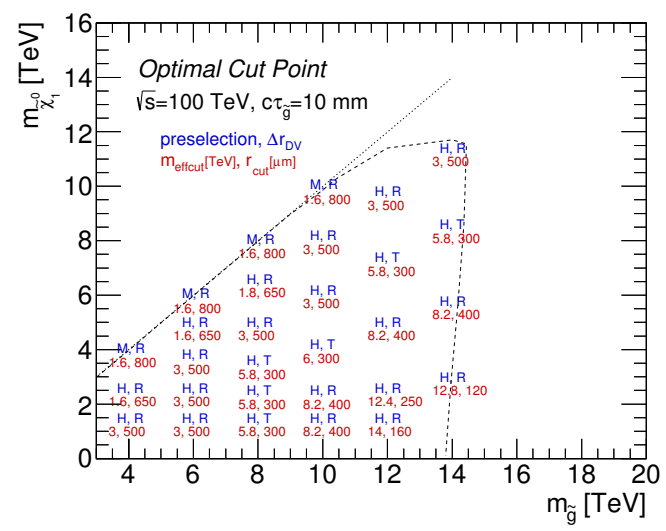

(f) $c \tau_{\tilde{g}}=10 \mathrm{~mm}$

Figure 20. Optimal cut values $\left(\left(m_{\text {effcut }}\right)_{\text {optimal }},\left(r_{\text {cut }}\right)_{\text {optimal }}\right)$, the preselection (L, M, H), and the discriminator $\Delta r_{\mathrm{DV}}(\mathrm{R}, \mathrm{T}, \mathrm{Z})$ for each sample point with various $c \tau_{\tilde{g}}$, with an integrated luminosity of $3000 \mathrm{fb}^{-1}$. The expected $5 \sigma$ discovery reaches for gluinos are also shown in the dashed lines. 


\begin{tabular}{|l|ccc|c|}
\hline & $Z$ & $W$ & $t \bar{t}$ & total \\
\hline preselection-H $\left(\times 10^{6}\right)$ & $0.42 \pm 0.01$ & $0.45 \pm 0.02$ & $1.26 \pm 0.4$ & $2.13 \pm 0.4$ \\
$m_{\text {eff }}$ (incl. $)>6 \mathrm{TeV}\left(\times 10^{3}\right)$ & $2.2 \pm 0.1$ & $1.0 \pm 0.1$ & $0.8 \pm 0.2$ & $4.0 \pm 0.3$ \\
\hline$\left|\boldsymbol{r}_{\mathrm{DV} 1}-\boldsymbol{r}_{\mathrm{DV} 2}\right|>200 \mu \mathrm{m}$ & $5.9 \pm 3$ & $5.7 \pm 2$. & $2.2 \pm 2$ & $14 \pm 4$ \\
$\left|\boldsymbol{r}_{\mathrm{DV} 1}-\boldsymbol{r}_{\mathrm{DV} 2}\right|>500 \mu \mathrm{m}$ & $<0.1$ & $<0.1$ & $<0.1$ & $<0.1$ \\
\hline
\end{tabular}

(a) The expected number of background events.

\begin{tabular}{|l|cccc|}
\hline & $c \tau_{\tilde{g}}=0$ & $c \tau_{\tilde{g}}=200 \mu \mathrm{m}$ & $c \tau_{\tilde{g}}=500 \mu \mathrm{m}$ & $c \tau_{\tilde{g}}=1 \mathrm{~mm}$ \\
\hline preselection-H & \multicolumn{4}{|c|}{$143 \pm 2$} \\
$m_{\mathrm{eff}}$ (incl.) $>6 \mathrm{TeV}$ & \multicolumn{4}{|c}{$70 \pm 1$} \\
\hline$\left|\boldsymbol{r}_{\mathrm{DV} 1}-\boldsymbol{r}_{\mathrm{DV} 2}\right|>200 \mu \mathrm{m}$ & $0.4 \pm 0.1$ & $26.5 \pm 0.7$ & $45.1 \pm 0.9$ & $53.5 \pm 1$ \\
$\left|\boldsymbol{r}_{\mathrm{DV} 1}-\boldsymbol{r}_{\mathrm{DV} 2}\right|>500 \mu \mathrm{m}$ & $<0.1$ & $6.4 \pm 0.3$ & $22.8 \pm 0.6$ & $36.5 \pm 0.8$ \\
\hline
\end{tabular}

(b) The expected number of signal events for $m_{\tilde{g}}=10 \mathrm{TeV}, m_{\tilde{\chi}_{1}^{0}}=8 \mathrm{TeV}$ with different values of $c \tau_{\tilde{g}}$.

Table 14. Same as table 13 but the masses of gluino and the LSP are set to be $10 \mathrm{TeV}$ and $8 \mathrm{TeV}$, respectively.

$12(10) \mathrm{TeV}$ and $m_{{\tilde{\chi_{1}^{0}}}^{0}}=100 \mathrm{GeV}(8 \mathrm{TeV})$. We then show the distributions of $m_{\text {eff }}$ (incl.) for the SM background and signal events with different values of $c \tau_{\tilde{g}}$ in figure 21 , with preselection-H and the vetoes in table 6 imposed. The masses of gluino and the LSP are set to be $14 \mathrm{TeV}$ and $100 \mathrm{GeV}$, respectively. Both the tables and figures demonstrate that also at a $100 \mathrm{TeV}$ collider our displaced-vertex selection cut can efficiently eliminate the SM background while keeping the signal events.

In figure 22a, we show the expected 95\% CL exclusion limits (in dotted lines) and $5 \sigma$ discovery reaches (in solid lines) for gluino as functions of $c \tau_{\tilde{g}}$ for different values of integrated luminosity at a $100 \mathrm{TeV}$ collider, where the LSP mass is set to be $100 \mathrm{GeV}$. The expected exclusion limit (discovery reach) for $c \tau_{\tilde{g}}=0$ is represented by a circle (a blob). As can be seen from the figure, the reach for the gluino can be extended with the help of the additional displaced-vertex cut for $c \tau_{\tilde{g}} \gtrsim 100 \mu \mathrm{m}$; for instance, for a gluino with $c \tau_{\tilde{g}} \sim \mathcal{O}(1-10) \mathrm{mm}$, the expected discovery reach for the gluino mass can be extended by as large as $\sim 1.4 \mathrm{TeV}(1.8 \mathrm{TeV})$ with an integrated luminosity of $\mathcal{L}=300 \mathrm{fb}^{-1}\left(3000 \mathrm{fb}^{-1}\right)$. These reaches for a gluino with $c \tau_{\tilde{g}} \sim \mathcal{O}(1-10) \mathrm{mm}$ are obtained with preselection-H, $\left|\boldsymbol{r}_{\mathrm{DV} 1}-\boldsymbol{r}_{\mathrm{DV} 2}\right|\left(\left|\boldsymbol{r}_{\mathrm{DV} 1}-\boldsymbol{r}_{\mathrm{DV} 2}\right|_{\mathrm{T}}\right)$ with $\left(m_{\text {effcut }}\right)_{\text {optimal }}=10 \mathrm{TeV}(12.6 \mathrm{TeV})$, and $\left(r_{\text {cut }}\right)_{\text {optimal }} \sim$ $120 \mu \mathrm{m}(160 \mu \mathrm{m})$ for $\mathcal{L}=300 \mathrm{fb}^{-1}\left(3000 \mathrm{fb}^{-1}\right)$. Compared to a promptly decaying gluino, where the optimized values for $\left(m_{\text {effcut }}\right)_{\text {optimal }}$ are given by $13.8 \mathrm{TeV}$ and $17.8 \mathrm{TeV}$ for $\mathcal{L}=300 \mathrm{fb}^{-1}$ and $3000 \mathrm{fb}^{-1}$, respectively, the $m_{\text {eff }}$ (incl.) selection cut is loosened and the new vertex-based selection cut is responsible for the reduction of background. We also show the degenerate case in figure $22 \mathrm{~b}$, where the mass difference between gluino and the LSP is set to be $100 \mathrm{GeV}$. We see a drastic enhancement in reaches, especially for $c \tau_{\tilde{g}} \sim \mathcal{O}(1-100) \mathrm{mm}$.

Finally, we show the $5 \sigma$ discovery reaches and expected 95\% CL exclusion limits for various values of $c \tau_{\tilde{g}}$ in figures 23 and 24 for an integrated luminosity of 300 and $3000 \mathrm{fb}^{-1}$, respectively. These figures illustrate that the use of our vertex-based selection 


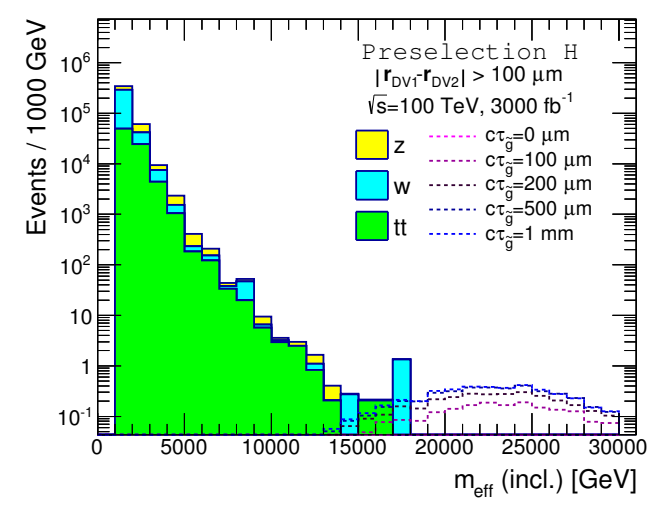

(a) $\left|\boldsymbol{r}_{\mathrm{DV} 1}-\boldsymbol{r}_{\mathrm{DV} 2}\right|>100 \mu \mathrm{m}$

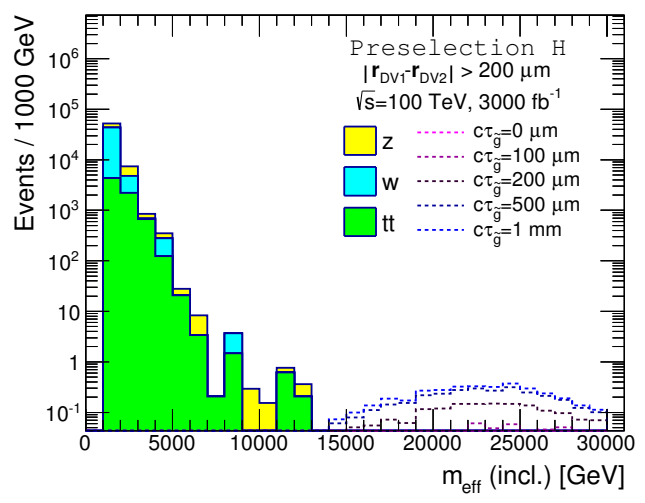

(b) $\left|\boldsymbol{r}_{\mathrm{DV} 1}-\boldsymbol{r}_{\mathrm{DV} 2}\right|>200 \mu \mathrm{m}$

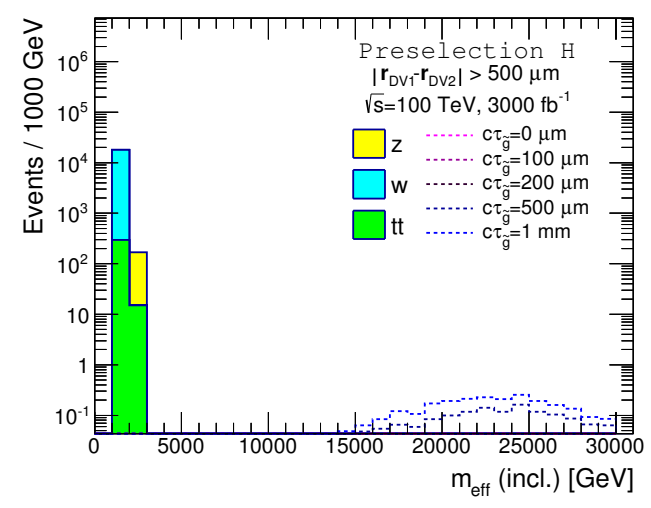

(c) $\left|\boldsymbol{r}_{\mathrm{DV} 1}-\boldsymbol{r}_{\mathrm{DV} 2}\right|>500 \mu \mathrm{m}$

Figure 21. Distributions of $m_{\mathrm{eff}}$ (incl.) for the SM background and signal events with different values of $c \tau_{\tilde{g}}$. The masses of gluino and the LSP are set to be $14 \mathrm{TeV}$ and $100 \mathrm{GeV}$, respectively. We have imposed preselection-H and the vetoes given in table 6 .

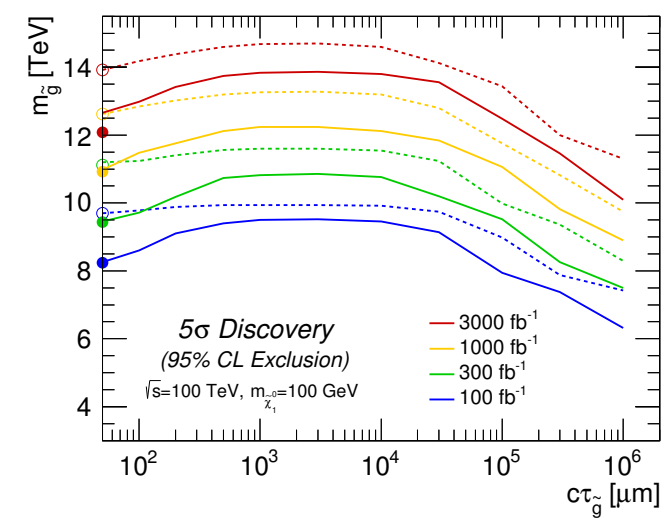

(a) $m_{\tilde{\chi}_{1}^{0}}=100 \mathrm{GeV}$

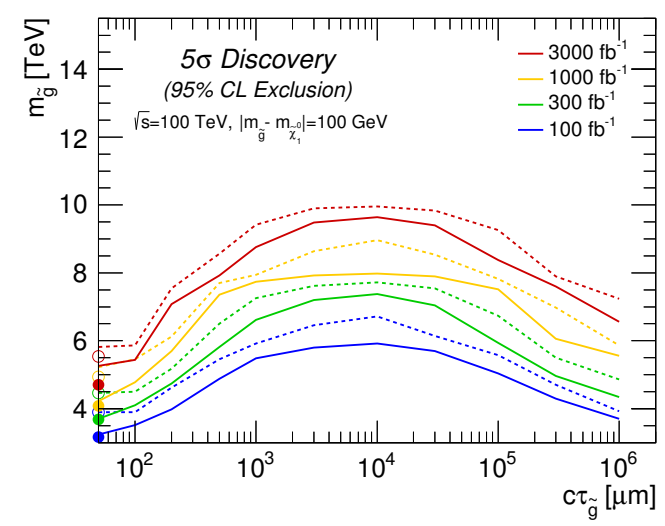

(b) $\left|m_{\tilde{g}}-m_{\tilde{\chi}_{1}^{0}}\right|=100 \mathrm{GeV}$

Figure 22. The expected $95 \%$ CL exclusion limits (dotted) and $5 \sigma$ discovery reaches (solid) as functions of $c \tau_{\tilde{g}}$ for different values of integrated luminosity at a future $100 \mathrm{TeV} p p$ collider. The expected exclusion limit (discovery reach) for $c \tau_{\tilde{g}}=0$ is represented by a circle (a blob). 


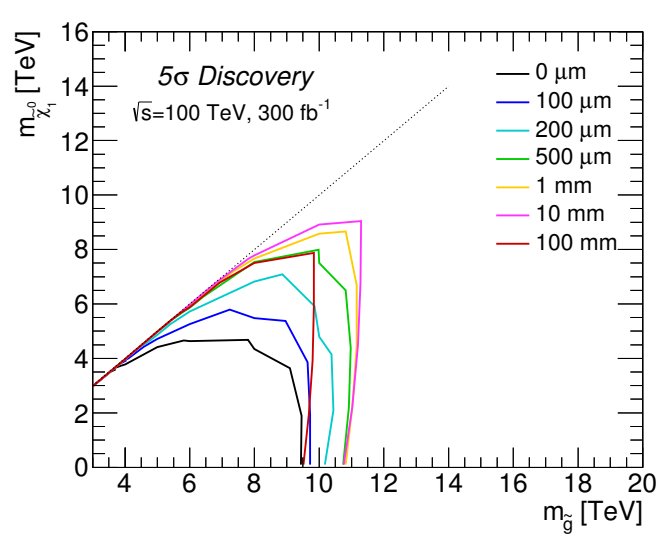

(a) $5 \sigma$ discovery

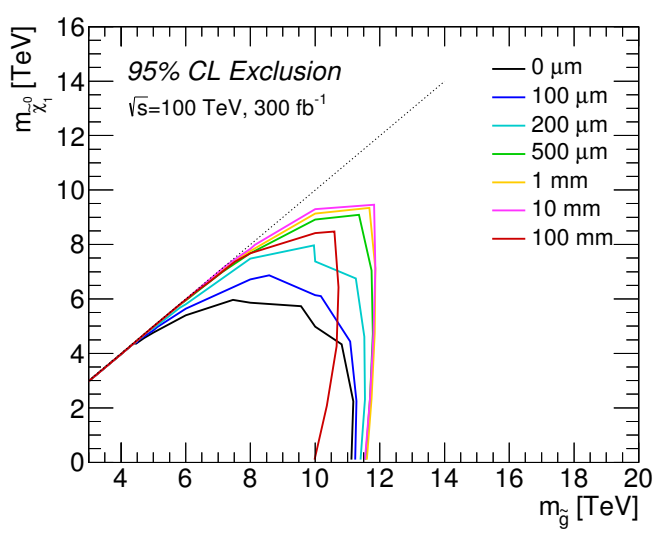

(b) Expected 95\% CL exclusion

Figure 23. $5 \sigma$ discovery reaches and expected 95\% CL exclusion limits for gluinos with different $c \tau_{\tilde{g}}$, for an integrated luminosity of $300 \mathrm{fb}^{-1}$.

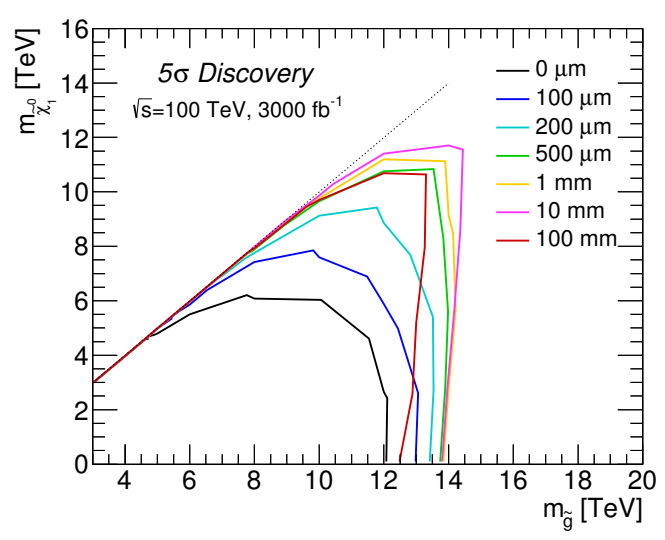

(a) $5 \sigma$ discovery

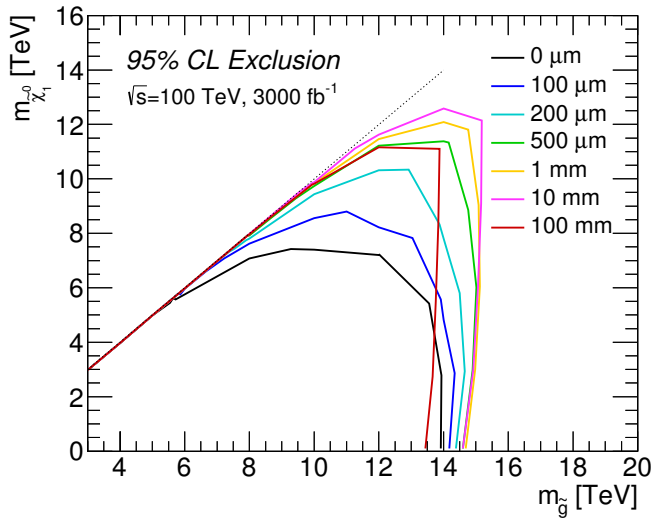

(b) Expected 95\% CL exclusion

Figure 24. Same as in figure 23, but for an integrated luminosity of $3000 \mathrm{fb}^{-1}$.

cut leads to a significant improvement in both the discovery reach and the exclusion limit for $c \tau_{\tilde{g}} \gtrsim 100 \mu \mathrm{m}$. Notice that, compared with the $13 \mathrm{TeV}$ case, searches at a $100 \mathrm{TeV}$ collider may be sensitive to gluinos with a shorter lifetime. The extent of the improvement is maximized for $c \tau_{\tilde{g}} \sim \mathcal{O}(1-10) \mathrm{mm}$ and tends to get larger for a heavier LSP, similarly to the $13 \mathrm{TeV}$ LHC case. We also find a dramatic improvement in the degenerate mass region; with the displaced-vertex selection cut, we may probe a gluino degenerate with the LSP in mass up to $\sim 10 \mathrm{TeV}$. This has significant implications for the gluino coannihilation scenario, given that an upper limit on the gluino mass is set for this scenario as $m_{\tilde{g}} \lesssim 8 \mathrm{TeV}[136]$. Our analysis indicates that we may probe the whole range of the gluino coannihilation scenario at a $100 \mathrm{TeV}$ collider if the gluino decay length falls into the range of $100 \mu \mathrm{m} \lesssim c \tau_{\tilde{g}} \lesssim 100 \mathrm{~mm}$. 


\section{Conclusion and discussion}

In this paper we have discussed a strategy of improving searches for metastable particles at hadron colliders. This strategy is based on the reconstruction of displaced vertices caused by the decay of metastable particles. We take account of this information as a new event-selection cut and impose this in addition to the conventional selection criteria based on kinematical observables. To see the significance of this new selection cut, we consider metastable gluinos in SUSY models as an example, whose decay length falls into the sub-millimeter range if squark masses are around the PeV scale. Then, we have studied the implications of this new selection cut for the gluino searches at both the $13 \mathrm{TeV} \mathrm{LHC}$ and future $100 \mathrm{TeV} p p$ collider experiments. We have performed MC simulations for both the signal and SM background events, and take into account the effect of the track reconstruction performance on the resolution of vertex reconstruction. We have also discussed optimization of kinematical selection criteria in the presence of the new vertex selection cut. As it turns out, we can considerably relax the kinematical selection criteria in this case, which is of great importance especially for the cases where gluino and the LSP are degenerate in mass.

For the $13 \mathrm{TeV}$ LHC analysis, we have found that our vertex-reconstruction method can separate out decay vertices if the gluino decay length is $\gtrsim 100 \mu \mathrm{m}$. As a result, with the displaced-vertex cut, we may considerably improve the potential of gluino searches for a gluino with $c \tau_{\tilde{g}} \gtrsim 200 \mu \mathrm{m}$. In particular, if $c \tau_{\tilde{g}} \sim \mathcal{O}(1-10) \mathrm{mm}$, then the exclusion and discovery reaches for the gluino mass can be extended by about $180 \mathrm{GeV}$ and $320 \mathrm{GeV}$, respectively, with an integrated luminosity of $3000 \mathrm{fb}^{-1}$ at the $13 \mathrm{TeV}$ LHC for LSP with a mass of $100 \mathrm{GeV}$. This improvement gets more drastic when gluino and the LSP are degenerate in mass. Furthermore, with an integrated luminosity of $3000 \mathrm{fb}^{-1}$, it is possible to measure the gluino decay length with an $\mathcal{O}(1)$ accuracy for a gluino with $c \tau_{\tilde{g}} \sim \mathcal{O}(100) \mu \mathrm{m}$ and $m_{\tilde{g}}=2.2 \mathrm{TeV}$, which may allow us to probe the $\mathrm{PeV}$-scale squarks indirectly.

After the discovery of a metastable particle, its lifetime information will become available by trying to reconstruct displaced vertices as we have seen in section 4.4. In the case of gluino, such information can be used to constrain the mass scale of squarks which mediate the decay processes. Even though we can only have an upper bound on the lifetime if the decay length $c \tau_{\tilde{g}}$ is shorter than $\sim 100 \mu \mathrm{m}$, such an bound is highly useful because it can provide an upper bound on the mass scale of squarks whose direct production may not be possible at the LHC. For the gluino mass of $2.2 \mathrm{TeV}$, for example, the mass scale of the squarks will be known to be lower than the $\mathrm{PeV}$ scale or a longevity of gluino will be observed.

We have also studied the prospects of searches for metastable gluinos at a future $100 \mathrm{TeV} p p$ collider. Since the TeV-scale gluinos tend to be produced in a fairly boosted state at a $100 \mathrm{TeV}$ collider, we expect it is possible to probe a shorter decay length compared with the LHC case. Indeed, we have found that the $\left|\boldsymbol{r}_{\mathrm{DV} 1}-\boldsymbol{r}_{\mathrm{DV} 2}\right|$ distributions for a $3 \mathrm{TeV}$ gluino are quite different from those for the $c \tau_{\tilde{g}}=0$ case if its decay distance is $\gtrsim 50 \mu \mathrm{m}$. By using the new selection cut, we can significantly extend the exclusion limits and discovery reaches of gluino searches for $c \tau_{\tilde{g}} \gtrsim 100 \mu \mathrm{m}$; e.g., for $c \tau_{\tilde{g}} \sim \mathcal{O}(1-10) \mathrm{mm}$, the exclusion 
and discovery reaches of gluino mass will be extended by about $780 \mathrm{GeV}$ and $1780 \mathrm{GeV}$, respectively, with an integrated luminosity of $3000 \mathrm{fb}^{-1}$ and the LSP mass being $100 \mathrm{GeV}$. The improvement is found to be more drastic in the degenerate mass region.

In the analyses given in this paper, we have assumed that gluinos decay into only the first-generation quarks and a LSP. In reality, the dominant decay channel of a gluino depends on the mass spectrum of squarks; for instance, if stops are much lighter than the other squarks, then the $\tilde{g} \rightarrow t \bar{t} \tilde{\chi}_{1}^{0}$ decay process becomes the dominant decay channel. In this case, besides the displaced vertices associated with the gluino decay, we may also find the secondary vertices that originate from long-lived hadrons including $b$ quarks, which are emitted by top quarks in the final state. Moreover, if there is a sizable flavor violation in the sfermion mass matrices - this possibility is experimentally allowed if the mass scale of SUSY particles lies around the PeV scale as we discussed in section 3- the decay of gluinos may exhibit this flavor mixing by containing quarks with different flavors in the final state [164]. Secondary vertices due to metastable hadrons may also appear in such cases. The presence of secondary vertices may affect the resolution of the vertex reconstruction, whereas this distinct signature composed of multiple displaced vertices may allow further optimization for this class of decay processes. A dedicated study regarding this possibility may be worth doing.

Another possibility of optimization is related to the degenerate mass region. As we observed in figure $11 \mathrm{~b}$ in section 4.3 , the limits we obtained in the degenerate mass region are weaker than that presented in ref. [144], since our analysis was not optimized to the degenerate mass region. Such an optimization is however possible by relaxing the requirements on the jet momenta. In fact, this is quite promising given that our new selection cut can significantly reduce the SM background and thus allow a relaxation of kinematical selection criteria as we have seen in the above analyses. This expectation is actually supported by the recent ATLAS result of the displaced-vertex search [67], where an optimization for the degenerate mass region was successfully carried out and a stringent limit is imposed on long-lived gluinos in a degenerate mass spectrum.

In this analysis, we do not reconstruct the position of the primary interaction point and use only the distance of the two displaced vertices as a discriminator. As we mentioned in section 4.2, however, the reconstruction of the primary vertex may also be possible by using the remnants of the $p p$ collision and/or initial state radiation emitted from the vertex. This additional information could be useful for the further reduction of background events. For instance, by requiring the presence of the primary vertex in-between the two reconstructed vertices we may efficiently reject the SM background contribution. Another, in fact simpler way of going beyond the mere use of the vertex distance is to require both of the two decay vertices to be reconstructed away from the beam line, with which we can avoid the misidintification of the primary vertex as one of the two decay vertices. Such elaboration of our new selection cut will be explored in another occasion.

Finally we comment here that, by reconstructing the positions of displaced vertices as well as the momenta of the charged tracks associated with these vertices, we may also extract the kinematical information of both the decaying and final-state invisible particles, as discussed in refs. $[165,166]$. In particular, we may determine the masses of these particles 
from the above information. This technique may be useful not only for studying the mass spectrum of the decay chain after the discovery of the metastable particle, but also for an additional event-selection cut to reduce the SM background. A dedicated study is required to assess the feasibility of this method, and thus we defer it to future work.

\section{Acknowledgments}

This work was supported in part by the Grant-in-Aid for Scientific Research C (26400239 $[\mathrm{TM}]$ ), Innovative Areas (16H06489 [OJ], 16H06490 [TM]), and Young Scentists B $(17 \mathrm{~K} 14270[\mathrm{NN}])$.

Open Access. This article is distributed under the terms of the Creative Commons Attribution License (CC-BY 4.0), which permits any use, distribution and reproduction in any medium, provided the original author(s) and source are credited.

\section{References}

[1] M. Fairbairn et al., Stable massive particles at colliders, Phys. Rept. 438 (2007) 1 [hep-ph/0611040] [INSPIRE].

[2] M. Toharia and J.D. Wells, Gluino decays with heavier scalar superpartners, JHEP 02 (2006) 015 [hep-ph/0503175] [INSPIRE].

[3] P. Gambino, G.F. Giudice and P. Slavich, Gluino decays in split supersymmetry, Nucl. Phys. B 726 (2005) 35 [hep-ph/0506214] [InSPIRE].

[4] R. Sato, S. Shirai and K. Tobioka, Gluino decay as a probe of high scale supersymmetry breaking, JHEP 11 (2012) 041 [arXiv:1207.3608] [INSPIRE].

[5] G.F. Giudice and R. Rattazzi, Theories with gauge mediated supersymmetry breaking, Phys. Rept. 322 (1999) 419 [hep-ph/9801271] [INSPIRE].

[6] P. Draper, P. Meade, M. Reece and D. Shih, Implications of a $125 \mathrm{GeV}$ Higgs for the MSSM and low-scale SUSY breaking, Phys. Rev. D 85 (2012) 095007 [arXiv:1112.3068] [INSPIRE].

[7] J.A. Evans and J. Shelton, Long-lived staus and displaced leptons at the LHC, JHEP 04 (2016) 056 [arXiv: 1601.01326] [inSPIRE].

[8] B.C. Allanach et al., Prompt signals and displaced vertices in sparticle searches for next-to-minimal gauge mediated supersymmetric models, Eur. Phys. J. C 76 (2016) 482 [arXiv: 1606.03099] [INSPIRE].

[9] R. Barbier et al., R-parity violating supersymmetry, Phys. Rept. 420 (2005) 1 [hep-ph/0406039] [INSPIRE].

[10] P.W. Graham, D.E. Kaplan, S. Rajendran and P. Saraswat, Displaced supersymmetry, JHEP 07 (2012) 149 [arXiv:1204.6038] [InSPIRE].

[11] P. Ghosh et al., Searching for left sneutrino LSP at the LHC, arXiv:1707.02471 [INSPIRE].

[12] A. Brandenburg et al., Signatures of axinos and gravitinos at colliders, Phys. Lett. B 617 (2005) 99 [hep-ph/0501287] [INSPIRE]. 
[13] K. Hamaguchi, M.M. Nojiri and A. de Roeck, Prospects to study a long-lived charged next lightest supersymmetric particle at the LHC, JHEP 03 (2007) 046 [hep-ph/0612060] [INSPIRE].

[14] A. Freitas, F.D. Steffen, N. Tajuddin and D. Wyler, Axinos in cosmology and at colliders, JHEP 06 (2011) 036 [arXiv:1105.1113] [inSPIRE].

[15] G. Barenboim, E.J. Chun, S. Jung and W.I. Park, Implications of an axino LSP for naturalness, Phys. Rev. D 90 (2014) 035020 [arXiv:1407.1218] [INSPIRE].

[16] C.S. Redino and D. Wackeroth, Exploring the hadronic axion window via delayed neutralino decay to axinos at the LHC, Phys. Rev. D 93 (2016) 075022 [arXiv:1512.06822] [INSPIRE].

[17] R.T. Co, F. D'Eramo and L.J. Hall, Gravitino or axino dark matter with reheat temperature as high as $10^{16} \mathrm{GeV}$, JHEP 03 (2017) 005 [arXiv: 1611.05028] [INSPIRE].

[18] R.T. Co, F. D'Eramo, L.J. Hall and K. Harigaya, Saxion cosmology for thermalized gravitino dark matter, JHEP 07 (2017) 125 [arXiv: 1703.09796] [INSPIRE].

[19] J. Fan, M. Reece and J.T. Ruderman, Stealth supersymmetry, JHEP 11 (2011) 012 [arXiv:1105.5135] [INSPIRE].

[20] J. Fan, M. Reece and J.T. Ruderman, A stealth supersymmetry sampler, JHEP 07 (2012) 196 [arXiv:1201.4875] [inSPIRE].

[21] J. Fan, R. Krall, D. Pinner, M. Reece and J.T. Ruderman, Stealth supersymmetry simplified, JHEP 07 (2016) 016 [arXiv:1512.05781] [INSPIRE].

[22] B. Batell, G.F. Giudice and M. McCullough, Natural heavy supersymmetry, JHEP 12 (2015) 162 [arXiv: 1509.00834] [InSPIRE].

[23] J.L. Evans, T. Gherghetta, N. Nagata and Z. Thomas, Naturalizing supersymmetry with a two-field relaxion mechanism, JHEP 09 (2016) 150 [arXiv: 1602.04812] [INSPIRE].

[24] J.L. Feng, T. Moroi, L. Randall, M. Strassler and S.-f. Su, Discovering supersymmetry at the Tevatron in wino LSP scenarios, Phys. Rev. Lett. 83 (1999) 1731 [hep-ph/9904250] [INSPIRE].

[25] M. Ibe, T. Moroi and T.T. Yanagida, Possible signals of wino LSP at the Large Hadron Collider, Phys. Lett. B 644 (2007) 355 [hep-ph/0610277] [INSPIRE].

[26] S. Asai, T. Moroi, K. Nishihara and T.T. Yanagida, Testing the anomaly mediation at the LHC, Phys. Lett. B 653 (2007) 81 [arXiv:0705.3086] [InSPIRE].

[27] S. Asai, T. Moroi and T.T. Yanagida, Test of anomaly mediation at the LHC, Phys. Lett. B 664 (2008) 185 [arXiv:0802.3725] [INSPIRE].

[28] S. Asai et al., Mass measurement of the decaying bino at the LHC, Phys. Lett. B 672 (2009) 339 [arXiv:0807.4987] [INSPIRE].

[29] Z. Liu and B. Tweedie, The fate of long-lived superparticles with hadronic decays after LHC Run 1, JHEP 06 (2015) 042 [arXiv: 1503.05923] [INSPIRE].

[30] N. Nagata, H. Otono and S. Shirai, Probing bino-gluino coannihilation at the LHC, Phys. Lett. B 748 (2015) 24 [arXiv: 1504.00504] [INSPIRE].

[31] N. Nagata, H. Otono and S. Shirai, Probing Bino-Wino coannihilation at the LHC, JHEP 10 (2015) 086 [arXiv:1506.08206] [INSPIRE]. 
[32] K. Rolbiecki and K. Sakurai, Long-lived bino and wino in supersymmetry with heavy scalars and higgsinos, JHEP 11 (2015) 091 [arXiv: 1506.08799] [INSPIRE].

[33] N. Nagata, H. Otono and S. Shirai, Cornering compressed gluino at the LHC, JHEP 03 (2017) 025 [arXiv: 1701.07664] [INSPIRE].

[34] H. Fukuda, N. Nagata, H. Otono and S. Shirai, Higgsino dark matter or not: role of disappearing track searches at the LHC and future colliders, Phys. Lett. B 781 (2018) 306 [arXiv:1703.09675] [INSPIRE].

[35] Z. Chacko, H.-S. Goh and R. Harnik, The twin Higgs: natural electroweak breaking from mirror symmetry, Phys. Rev. Lett. 96 (2006) 231802 [hep-ph/0506256] [INSPIRE].

[36] G. Burdman, Z. Chacko, H.-S. Goh and R. Harnik, Folded supersymmetry and the LEP paradox, JHEP 02 (2007) 009 [hep-ph/0609152] [INSPIRE].

[37] H. Cai, H.-C. Cheng and J. Terning, A Quirky Little Higgs Model, JHEP 05 (2009) 045 [arXiv: 0812.0843] [inSPIRE].

[38] G. Burdman and R.T. D'Agnolo, Scalar leptons in folded supersymmetry, arXiv: 1512.00040 [INSPIRE].

[39] Z. Chacko, D. Curtin and C.B. Verhaaren, A quirky probe of neutral naturalness, Phys. Rev. D 94 (2016) 011504 [arXiv: 1512.05782] [INSPIRE].

[40] M.J. Strassler and K.M. Zurek, Echoes of a hidden valley at hadron colliders, Phys. Lett. B 651 (2007) 374 [hep-ph/0604261] [INSPIRE].

[41] M.J. Strassler and K.M. Zurek, Discovering the Higgs through highly-displaced vertices, Phys. Lett. B 661 (2008) 263 [hep-ph/0605193] [INSPIRE].

[42] M.J. Strassler, Possible effects of a hidden valley on supersymmetric phenomenology, hep-ph/0607160 [INSPIRE].

[43] Y. Nakai, M. Reece and R. Sato, SUSY Higgs mass and collider signals with a hidden valley, JHEP 03 (2016) 143 [arXiv:1511.00691] [INSPIRE].

[44] S. Knapen, H.K. Lou, M. Papucci and J. Setford, Tracking down quirks at the Large Hadron Collider, Phys. Rev. D 96 (2017) 115015 [arXiv:1708.02243] [InSPIRE].

[45] J. Barnard, P. Cox, T. Gherghetta and A. Spray, Long-lived, colour-triplet scalars from unnaturalness, JHEP 03 (2016) 003 [arXiv:1510.06405] [INSPIRE].

[46] S. Chang and M.A. Luty, Displaced dark matter at colliders, arXiv:0906.5013 [INSPIRE].

[47] R.T. Co, F. D'Eramo, L.J. Hall and D. Pappadopulo, Freeze-in dark matter with displaced signatures at colliders, JCAP 12 (2015) 024 [arXiv: 1506.07532] [INSPIRE].

[48] O. Buchmueller et al., Simplified models for displaced dark matter signatures, JHEP 09 (2017) 076 [arXiv: 1704.06515] [INSPIRE].

[49] B.S. Acharya et al., Categorisation and detection of dark matter candidates from string/M-theory hidden sectors, arXiv:1707.04530 [INSPIRE].

[50] L. Basso, A. Belyaev, S. Moretti and C.H. Shepherd-Themistocleous, Phenomenology of the minimal B-L extension of the Standard model: $Z^{\prime}$ and neutrinos, Phys. Rev. D 80 (2009) 055030 [arXiv: 0812.4313] [INSPIRE].

[51] J.C. Helo, M. Hirsch and S. Kovalenko, Heavy neutrino searches at the LHC with displaced vertices, Phys. Rev. D 89 (2014) 073005 [arXiv:1312.2900] [InSPIRE]. 
[52] E. Izaguirre and B. Shuve, Multilepton and lepton jet probes of sub-weak-scale right-handed neutrinos, Phys. Rev. D 91 (2015) 093010 [arXiv:1504.02470] [InSPIRE].

[53] A. Maiezza, M. Nemevšek and F. Nesti, Lepton number violation in Higgs decay at LHC, Phys. Rev. Lett. 115 (2015) 081802 [arXiv:1503.06834] [INSPIRE].

[54] S. Antusch, E. Cazzato and O. Fischer, Displaced vertex searches for sterile neutrinos at future lepton colliders, JHEP 12 (2016) 007 [arXiv: 1604.02420] [INSPIRE].

[55] S. Antusch, E. Cazzato and O. Fischer, Sterile neutrino searches at future $e^{-} e^{+}$, pp and $e^{-} p$ colliders, Int. J. Mod. Phys. A 32 (2017) 1750078 [arXiv:1612.02728] [inSPIRE].

[56] E. Accomando et al., Novel SM-like Higgs decay into displaced heavy neutrino pairs in U(1)' models, JHEP 04 (2017) 081 [arXiv: 1612.05977] [INSPIRE].

[57] M. Nemevšek, F. Nesti and J.C. Vasquez, Majorana higgses at colliders, JHEP 04 (2017) 114 [arXiv: 1612.06840] [INSPIRE].

[58] P. Bhupal Dev, R.N. Mohapatra and Y. Zhang, Displaced photon signal from a possible light scalar in minimal left-right seesaw model, Phys. Rev. D 95 (2017) 115001 [arXiv: 1612.09587] [INSPIRE].

[59] G. Cottin, J.C. Helo and M. Hirsch, Searches for light sterile neutrinos with multitrack displaced vertices, Phys. Rev. D 97 (2018) 055025 [arXiv: 1801.02734] [INSPIRE].

[60] ATLAS collaboration, Search for long-lived neutral particles decaying into lepton jets in proton-proton collisions at $\sqrt{s}=8 \mathrm{TeV}$ with the ATLAS detector, JHEP 11 (2014) 088 [arXiv: 1409.0746] [INSPIRE].

[61] CMS collaboration, Search for long-lived neutral particles decaying to quark-antiquark pairs in proton-proton collisions at $\sqrt{s}=8$ TeV, Phys. Rev. D 91 (2015) 012007 [arXiv:1411.6530] [INSPIRE].

[62] CMS collaboration, Search for long-lived particles that decay into final states containing two electrons or two muons in proton-proton collisions at $\sqrt{s}=8$ TeV, Phys. Rev. D 91 (2015) 052012 [arXiv:1411.6977] [INSPIRE].

[63] LHCb collaboration, Search for long-lived particles decaying to jet pairs, Eur. Phys. J. C 75 (2015) 152 [arXiv: 1412.3021] [INSPIRE].

[64] ATLAS collaboration, Search for long-lived, weakly interacting particles that decay to displaced hadronic jets in proton-proton collisions at $\sqrt{s}=8 \mathrm{TeV}$ with the ATLAS detector, Phys. Rev. D 92 (2015) 012010 [arXiv:1504.03634] [InSPIRE].

[65] ATLAS collaboration, Search for massive, long-lived particles using multitrack displaced vertices or displaced lepton pairs in pp collisions at $\sqrt{s}=8 \mathrm{TeV}$ with the ATLAS detector, Phys. Rev. D 92 (2015) 072004 [arXiv: 1504.05162] [INSPIRE].

[66] CMS collaboration, Search for R-parity violating supersymmetry with displaced vertices in proton-proton collisions at $\sqrt{s}=8$ TeV, Phys. Rev. D 95 (2017) 012009

[arXiv: 1610.05133] [INSPIRE].

[67] ATLAS collaboration, Search for long-lived, massive particles in events with displaced vertices and missing transverse momentum in $\sqrt{s}=13 \mathrm{TeV}$ pp collisions with the ATLAS detector, Phys. Rev. D 97 (2018) 052012 [arXiv: 1710.04901] [INSPIRE].

[68] ATLAS collaboration, Search for charginos nearly mass degenerate with the lightest 
neutralino based on a disappearing-track signature in pp collisions at $\sqrt{s}=8 \mathrm{TeV}$ with the ATLAS detector, Phys. Rev. D 88 (2013) 112006 [arXiv: 1310.3675] [INSPIRE].

[69] CMS collaboration, Search for disappearing tracks in proton-proton collisions at $\sqrt{s}=8$ TeV, JHEP 01 (2015) 096 [arXiv: 1411.6006] [INSPIRE].

[70] ATLAS collaboration, Search for long-lived charginos based on a disappearing-track signature in pp collisions at $\sqrt{s}=13 \mathrm{TeV}$ with the ATLAS detector, JHEP 06 (2018) 022 [arXiv: 1712.02118] [INSPIRE].

[71] ATLAS collaboration, Searches for heavy long-lived charged particles with the ATLAS detector in proton-proton collisions at $\sqrt{s}=8 \mathrm{TeV}$, JHEP 01 (2015) 068 [arXiv:1411.6795] [INSPIRE].

[72] ATLAS collaboration, Search for metastable heavy charged particles with large ionisation energy loss in pp collisions at $\sqrt{s}=8 \mathrm{TeV}$ using the ATLAS experiment, Eur. Phys. J. C 75 (2015) 407 [arXiv: 1506.05332] [INSPIRE].

[73] ATLAS collaboration, Search for metastable heavy charged particles with large ionization energy loss in pp collisions at $\sqrt{s}=13$ TeV using the ATLAS experiment, Phys. Rev. D 93 (2016) 112015 [arXiv: 1604.04520] [inSPIRE].

[74] ATLAS collaboration, Search for heavy long-lived charged $R$-hadrons with the ATLAS detector in $3.2 \mathrm{fb}^{-1}$ of proton-proton collision data at $\sqrt{s}=13 \mathrm{TeV}$, Phys. Lett. B 760 (2016) 647 [arXiv: 1606.05129] [INSPIRE].

[75] CMS collaboration, Search for long-lived charged particles in proton-proton collisions at $\sqrt{s}=13$ TeV, Phys. Rev. D 94 (2016) 112004 [arXiv:1609.08382] [inSPIRE].

[76] CMS collaboration, Search for displaced supersymmetry in events with an electron and a muon with large impact parameters, Phys. Rev. Lett. 114 (2015) 061801 [arXiv:1409.4789] [INSPIRE].

[77] CMS collaboration, Search for displaced leptons in the e-mu channel, CMS-PAS-EXO-16-022 (2016).

[78] H. Ito, O. Jinnouchi, T. Moroi, N. Nagata and H. Otono, Extending the LHC reach for new physics with sub-millimeter displaced vertices, Phys. Lett. B 771 (2017) 568 [arXiv: 1702.08613] [INSPIRE].

[79] N. Arkani-Hamed, T. Han, M. Mangano and L.-T. Wang, Physics opportunities of a $100 \mathrm{TeV}$ proton-proton collider, Phys. Rept. 652 (2016) 1 [arXiv:1511.06495] [INSPIRE].

[80] T. Golling et al., Physics at a $100 \mathrm{TeV}$ pp collider: beyond the standard model phenomena, CERN Yellow Report (2017) 441 [arXiv: 1606. 00947] [INSPIRE].

[81] ATLAS collaboration, Performance of primary vertex reconstruction in proton-proton collisions at $\sqrt{s}=7 \mathrm{TeV}$ in the ATLAS experiment, ATLAS-CONF-2010-069 (2010).

[82] ATLAS collaboration, Reconstruction of primary vertices at the ATLAS experiment in Run 1 proton-proton collisions at the LHC, Eur. Phys. J. C 77 (2017) 332 [arXiv:1611.10235] [INSPIRE].

[83] ATLAS collaboration, Vertex reconstruction performance of the ATLAS detector at $\sqrt{s}=13 \mathrm{TeV}$, ATL-PHYS-PUB-2015-026 (2015).

[84] CMS collaboration, Description and performance of track and primary-vertex reconstruction with the CMS tracker, 2014 JINST 9 P10009 [arXiv:1405.6569] [INSPIRE]. 
[85] CMS collaboration, Primary vertex resolution in 2016, CMS-DP-2016-041 (2016).

[86] R. Fruhwirth, W. Waltenberger and P. Vanlaer, Adaptive vertex fitting, J. Phys. G 34 (2007) N343 [INSPIRE].

[87] D.R. Bickel and R. Fruhwirth, On a fast, robust estimator of the mode: comparisons to other robust estimators with applications, Comput. Stat. Data Anal. 50 (2006) 3500 [math/0505419].

[88] ATLAS collaboration, Impact parameter resolution, https://atlas.web.cern.ch/Atlas/GROUPS/PHYSICS/PLOTS/IDTR-2015-007/ (2015).

[89] ATLAS collaboration, Track reconstruction performance of the ATLAS inner detector at $\sqrt{s}=13$ TeV, ATL-PHYS-PUB-2015-018 (2015).

[90] ATLAS collaboration, The ATLAS experiment at the CERN Large Hadron Collider, 2008 JINST 3 S08003 [INSPIRE].

[91] ATLAS collaboration, Impact parameter resolution using $2016 \mathrm{MB}$ data, https://atlas.web.cern.ch/Atlas/GROUPS/PHYSICS/PLOTS/IDTR-2016-018/ (2016).

[92] ATLAS, CMS collaboration, Combined measurement of the Higgs boson mass in pp collisions at $\sqrt{s}=7$ and 8 TeV with the ATLAS and CMS Experiments, Phys. Rev. Lett. 114 (2015) 191803 [arXiv: 1503.07589] [INSPIRE].

[93] Y. Okada, M. Yamaguchi and T. Yanagida, Upper bound of the lightest Higgs boson mass in the minimal supersymmetric standard model, Prog. Theor. Phys. 85 (1991) 1 [InSPIRE].

[94] Y. Okada, M. Yamaguchi and T. Yanagida, Renormalization group analysis on the Higgs mass in the softly broken supersymmetric standard model, Phys. Lett. B 262 (1991) 54 [INSPIRE].

[95] J.R. Ellis, G. Ridolfi and F. Zwirner, Radiative corrections to the masses of supersymmetric Higgs bosons, Phys. Lett. B 257 (1991) 83 [INSPIRE].

[96] J.R. Ellis, G. Ridolfi and F. Zwirner, On radiative corrections to supersymmetric Higgs boson masses and their implications for LEP searches, Phys. Lett. B 262 (1991) 477 [INSPIRE].

[97] H.E. Haber and R. Hempfling, Can the mass of the lightest Higgs boson of the minimal supersymmetric model be larger than $m(Z)$ ?, Phys. Rev. Lett. 66 (1991) 1815 [INSPIRE].

[98] F. Gabbiani, E. Gabrielli, A. Masiero and L. Silvestrini, A complete analysis of FCNC and CP constraints in general SUSY extensions of the standard model, Nucl. Phys. B 477 (1996) 321 [hep-ph/9604387] [INSPIRE].

[99] T. Moroi and M. Nagai, Probing supersymmetric model with heavy sfermions using leptonic flavor and CP-violations, Phys. Lett. B 723 (2013) 107 [arXiv:1303.0668] [InSPIRE].

[100] D. McKeen, M. Pospelov and A. Ritz, Electric dipole moment signatures of PeV-scale superpartners, Phys. Rev. D 87 (2013) 113002 [arXiv:1303.1172] [INSPIRE].

[101] W. Altmannshofer, R. Harnik and J. Zupan, Low energy probes of PeV scale sfermions, JHEP 11 (2013) 202 [arXiv:1308.3653] [INSPIRE].

[102] K. Fuyuto, J. Hisano, N. Nagata and K. Tsumura, QCD corrections to quark (chromo)electric dipole moments in high-scale supersymmetry, JHEP 12 (2013) 010 [arXiv:1308.6493] [INSPIRE]. 
[103] J. Hisano, D. Kobayashi, T. Kuwahara and N. Nagata, Decoupling Can Revive Minimal Supersymmetric SU(5), JHEP 07 (2013) 038 [arXiv: 1304.3651] [INSPIRE].

[104] N. Nagata and S. Shirai, Sfermion flavor and proton decay in high-scale supersymmetry, JHEP 03 (2014) 049 [arXiv:1312.7854] [inSPIRE].

[105] J.L. Evans, N. Nagata and K.A. Olive, SU(5) grand unification in pure gravity mediation, Phys. Rev. D 91 (2015) 055027 [arXiv: 1502.00034] [InSPIRE].

[106] G.F. Giudice, M.A. Luty, H. Murayama and R. Rattazzi, Gaugino mass without singlets, JHEP 12 (1998) 027 [hep-ph/9810442] [INSPIRE].

[107] J.D. Wells, Implications of supersymmetry breaking with a little hierarchy between gauginos and scalars, talk given at the $11^{\text {th }}$ International Conference on Supersymmetry and the Unification of Fundamental Interactions (SUSY 2003), JUne 5-10, Tucson, Arizona U.S.A. (2003), hep-ph/0306127 [INSPIRE].

[108] N. Arkani-Hamed and S. Dimopoulos, Supersymmetric unification without low energy supersymmetry and signatures for fine-tuning at the LHC, JHEP 06 (2005) 073 [hep-th/0405159] [INSPIRE].

[109] G.F. Giudice and A. Romanino, Split supersymmetry, Nucl. Phys. B 699 (2004) 65 [Erratum ibid. B 706 (2005) 487] [hep-ph/0406088] [INSPIRE].

[110] N. Arkani-Hamed, S. Dimopoulos, G.F. Giudice and A. Romanino, Aspects of split supersymmetry, Nucl. Phys. B 709 (2005) 3 [hep-ph/0409232] [INSPIRE].

[111] J.D. Wells, PeV-scale supersymmetry, Phys. Rev. D 71 (2005) 015013 [hep-ph/0411041] [INSPIRE].

[112] L. Randall and R. Sundrum, Out of this world supersymmetry breaking, Nucl. Phys. B 557 (1999) 79 [hep-th/9810155] [INSPIRE].

[113] T. Gherghetta, G.F. Giudice and J.D. Wells, Phenomenological consequences of supersymmetry with anomaly induced masses, Nucl. Phys. B 559 (1999) 27 [hep-ph/9904378] [INSPIRE].

[114] T. Moroi and L. Randall, Wino cold dark matter from anomaly mediated SUSY breaking, Nucl. Phys. B 570 (2000) 455 [hep-ph/9906527] [INSPIRE].

[115] L.J. Hall and Y. Nomura, Spread supersymmetry, JHEP 01 (2012) 082 [arXiv:1111.4519] [INSPIRE].

[116] M. Ibe and T.T. Yanagida, The lightest Higgs boson mass in pure gravity mediation model, Phys. Lett. B 709 (2012) 374 [arXiv:1112.2462] [INSPIRE].

[117] M. Ibe, S. Matsumoto and T.T. Yanagida, Pure gravity mediation with $m_{3 / 2}=10-100 \mathrm{TeV}$, Phys. Rev. D 85 (2012) 095011 [arXiv: 1202.2253] [InSPIRE].

[118] A. Arvanitaki, N. Craig, S. Dimopoulos and G. Villadoro, Mini-split, JHEP 02 (2013) 126 [arXiv: 1210.0555] [INSPIRE].

[119] L.J. Hall, Y. Nomura and S. Shirai, Spread supersymmetry with Wino LSP: gluino and dark matter signals, JHEP 01 (2013) 036 [arXiv:1210.2395] [INSPIRE].

[120] N. Arkani-Hamed et al., Simply unnatural supersymmetry, arXiv:1212.6971 [INSPIRE].

[121] J.L. Evans, M. Ibe, K.A. Olive and T.T. Yanagida, Universality in pure gravity mediation, Eur. Phys. J. C 73 (2013) 2468 [arXiv:1302.5346] [InSPIRE]. 
[122] J.L. Evans, K.A. Olive, M. Ibe and T.T. Yanagida, Non-universalities in pure gravity mediation, Eur. Phys. J. C 73 (2013) 2611 [arXiv:1305.7461] [INSPIRE].

[123] D.M. Pierce, J.A. Bagger, K.T. Matchev and R.-j. Zhang, Precision corrections in the minimal supersymmetric standard model, Nucl. Phys. B 491 (1997) 3 [hep-ph/9606211] [INSPIRE].

[124] A. Pomarol and R. Rattazzi, Sparticle masses from the superconformal anomaly, JHEP 05 (1999) 013 [hep-ph/9903448] [INSPIRE].

[125] A.E. Nelson and N.J. Weiner, Extended anomaly mediation and new physics at 10-TeV, hep-ph/0210288 [INSPIRE].

[126] K. Hsieh and M.A. Luty, Mixed gauge and anomaly mediation from new physics at 10-TeV, JHEP 06 (2007) 062 [hep-ph/0604256] [INSPIRE].

[127] A. Gupta, D.E. Kaplan and T. Zorawski, Gaugomaly mediation revisited, JHEP 11 (2013) 149 [arXiv:1212.6969] [INSPIRE].

[128] K. Nakayama and T.T. Yanagida, Anomaly mediation deformed by axion, Phys. Lett. B 722 (2013) 107 [arXiv: 1302.3332] [INSPIRE].

[129] K. Harigaya, M. Ibe and T.T. Yanagida, A closer look at gaugino masses in pure gravity mediation model/minimal split SUSY model, JHEP 12 (2013) 016 [arXiv:1310.0643] [INSPIRE].

[130] J.L. Evans and K.A. Olive, Universality in pure gravity mediation with vector multiplets, Phys. Rev. D 90 (2014) 115020 [arXiv:1408.5102] [INSPIRE].

[131] K. Griest and D. Seckel, Three exceptions in the calculation of relic abundances, Phys. Rev. D 43 (1991) 3191 [INSPIRE].

[132] S. Profumo and C.E. Yaguna, Gluino coannihilations and heavy bino dark matter, Phys. Rev. D 69 (2004) 115009 [hep-ph/0402208] [INSPIRE].

[133] D. Feldman, Z. Liu and P. Nath, Gluino NLSP, dark matter via gluino coannihilation and LHC signatures, Phys. Rev. D 80 (2009) 015007 [arXiv: 0905.1148] [InSPIRE].

[134] A. De Simone, G.F. Giudice and A. Strumia, Benchmarks for dark matter searches at the LHC, JHEP 06 (2014) 081 [arXiv: 1402.6287] [INSPIRE].

[135] K. Harigaya, K. Kaneta and S. Matsumoto, Gaugino coannihilations, Phys. Rev. D 89 (2014) 115021 [arXiv:1403.0715] [INSPIRE].

[136] J. Ellis, F. Luo and K.A. Olive, Gluino Coannihilation Revisited, JHEP 09 (2015) 127 [arXiv: 1503.07142] [INSPIRE].

[137] J. Ellis, J.L. Evans, F. Luo and K.A. Olive, Scenarios for gluino coannihilation, JHEP 02 (2016) 071 [arXiv: 1510.03498] [INSPIRE].

[138] S.P. Liew and F. Luo, Effects of QCD bound states on dark matter relic abundance, JHEP 02 (2017) 091 [arXiv: 1611.08133] [INSPIRE].

[139] P.M. Nadolsky et al., Implications of CTEQ global analysis for collider observables, Phys. Rev. D 78 (2008) 013004 [arXiv: 0802.0007] [InSPIRE].

[140] C. Borschensky et al., Squark and gluino production cross sections in pp collisions at $\sqrt{s}=13,14,33$ and $100 \mathrm{TeV}$, Eur. Phys. J. C 74 (2014) 3174 [arXiv:1407.5066] [INSPIRE]. 
[141] J. Alwall et al., The automated computation of tree-level and next-to-leading order differential cross sections and their matching to parton shower simulations, JHEP 07 (2014) 079 [arXiv : 1405.0301] [inSPIRE].

[142] T. Sjöstrand, S. Mrenna and P.Z. Skands, A brief introduction to PYTHIA 8.1, Comput. Phys. Commun. 178 (2008) 852 [arXiv:0710.3820] [InSPIRE].

[143] J.D. Bjorken, Properties of hadron distributions in reactions containing very heavy quarks, Phys. Rev. D 17 (1978) 171 [inSPIRE].

[144] ATLAS collaboration, Search for squarks and gluinos in final states with jets and missing transverse momentum using $36 \mathrm{fb}^{-1}$ of $\sqrt{\mathrm{s}}=13 \mathrm{TeV}$ pp collision data with the ATLAS detector, Phys. Rev. D 97 (2018) 112001 [arXiv:1712.02332] [INSPIRE].

[145] T. Cohen et al., SUSY simplified models at 14, 33 and 100 TeV proton colliders, JHEP 04 (2014) 117 [arXiv:1311.6480] [INSPIRE].

[146] J. Alwall et al., Comparative study of various algorithms for the merging of parton showers and matrix elements in hadronic collisions, Eur. Phys. J. C 53 (2008) 473 [arXiv:0706.2569] [INSPIRE].

[147] A. Avetisyan et al., Methods and results for standard model event generation at $\sqrt{s}=14$ $\mathrm{TeV}, 33 \mathrm{TeV}$ and $100 \mathrm{TeV}$ Proton Colliders (a Snowmass whitepaper), in the proceedings of the 2013 Community Summer Study on the Future of U.S. Particle Physics: Snowmass on the Mississippi (CSS2013), July 29-August 6, Minneapolis, U.S.A. (2013), arXiv:1308.1636 [INSPIRE].

[148] DELPHES 3 collaboration, J. de Favereau et al., DELPHES 3, A modular framework for fast simulation of a generic collider experiment, JHEP 02 (2014) 057 [arXiv:1307.6346] [INSPIRE].

[149] M. Cacciari, G.P. Salam and G. Soyez, FastJet user manual, Eur. Phys. J. C 72 (2012) 1896 [arXiv:1111.6097] [INSPIRE].

[150] J.D. Bjorken and S.J. Brodsky, Statistical model for electron-positron annihilation into hadrons, Phys. Rev. D 1 (1970) 1416 [INSPIRE].

[151] C. Chen, New approach to identifying boosted hadronically-decaying particle using jet substructure in its center-of-mass frame, Phys. Rev. D 85 (2012) 034007 [arXiv:1112.2567] [INSPIRE].

[152] ATLAS collaboration, ATLAS insertable b-layer technical design report, CERN-LHCC-2010-013 (2010).

[153] A. Miucci, The ATLAS insertable b-layer project, 2014 JINST 9 C02018.

[154] G.A.M. Aaboud et al., A measurement of material in the atlas tracker using secondary hadronic interactions in 7 tev pp collisions, 2016 JINST 11 P11020.

[155] A.L. Read, Presentation of search results: the CL technique, J. Phys. G 28 (2002) 2693 [INSPIRE].

[156] ATLAS collaboration, Technical design report for the ATLAS inner tracker strip detector, CERN-LHCC-2017-005 (2017).

[157] T. Junk, Confidence level computation for combining searches with small statistics, Nucl. Instrum. Meth. A 434 (1999) 435 [hep-ex/9902006] [INSPIRE]. 
[158] G. Cowan, K. Cranmer, E. Gross and O. Vitells, Asymptotic formulae for likelihood-based tests of new physics, Eur. Phys. J. C 71 (2011) 1554 [Erratum ibid. C 73 (2013) 2501] [arXiv: 1007.1727] [INSPIRE].

[159] ATLAS collaboration, Limits on metastable gluinos from ATLAS SUSY searches at $8 \mathrm{TeV}$, ATLAS-CONF-2014-037 (2014).

[160] CMS collaboration, Search for natural and split supersymmetry in proton-proton collisions at $\sqrt{s}=13 \mathrm{TeV}$ in final states with jets and missing transverse momentum, JHEP 05 (2018) 025 [arXiv: 1802.02110] [INSPIRE].

[161] CMS collaboration, Identification of heavy-flavour jets with the CMS detector in $p p$ collisions at $13 \mathrm{TeV}, 2018$ JINST 13 P05011 [arXiv:1712.07158] [INSPIRE].

[162] ATLAS collaboration, Further searches for squarks and gluinos in final states with jets and missing transverse momentum at $\sqrt{s}=13 \mathrm{TeV}$ with the ATLAS detector, ATLAS-CONF-2016-078 (2016).

[163] S.A.R. Ellis and B. Zheng, Reaching for squarks and gauginos at a $100 \mathrm{TeV}$ pp collider, Phys. Rev. D 92 (2015) 075034 [arXiv:1506.02644] [INSPIRE].

[164] R. Sato, S. Shirai and K. Tobioka, Flavor of gluino decay in high-scale supersymmetry, JHEP 10 (2013) 157 [arXiv:1307.7144] [INSPIRE].

[165] M. Park and Y. Zhao, Recovering particle masses from missing energy signatures with displaced tracks, arXiv:1110.1403 [INSPIRE].

[166] G. Cottin, Reconstructing particle masses in events with displaced vertices, JHEP 03 (2018) 137 [arXiv: 1801.09671$]$ [INSPIRE]. 